\title{
Submillimeter Galaxies
}

\author{
Andrew W. Blain ${ }^{\mathrm{a}, \mathrm{b}, 1}$, Ian Smail ${ }^{\mathrm{c}}$, R. J. Ivison ${ }^{\mathrm{d}}$, J.-P. Kneib ${ }^{\mathrm{e}}$, \\ David T. Frayer ${ }^{\mathrm{f}}$

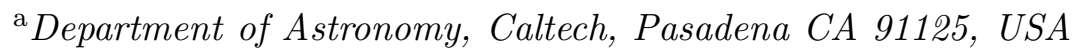 \\ ${ }^{\mathrm{b}}$ Institute of Astronomy, Madingley Road, Cambridge CBз OHA, UK \\ ${ }^{\mathrm{c}}$ Department of Physics, University of Durham, South Road, Durham DH1 3LE, \\ $U K$ \\ ${ }^{\mathrm{d}}$ Institute for Astronomy, University of Edinburgh, Edinburgh EH9 3HJ, UK \\ e Observatoire Midi-Pyrénées, 14 Avenue E. Belin, F-31400 Toulouse, France \\ ${ }^{\mathrm{f}}$ SIRTF Science Center, Caltech, Pasadena CA 91125, USA
}

\begin{abstract}
A cosmologically significant population of very luminous high-redshift galaxies has recently been discovered at submillimeter (submm) wavelengths. Advances in submm detector technologies have opened this new window on the distant Universe. Here we discuss the properties of the high-redshift submm galaxies, their significance for our understanding of the process of galaxy formation, and the selection effects that apply to deep submm surveys. The submm galaxies generate a significant fraction of the energy output of all the galaxies in the early Universe. We emphasize the importance of studying a complete sample of submm galaxies, and stress that because they are typically very faint in other wavebands, these follow-up observations are very challenging. Finally, we discuss the surveys that will be made using the next generation of submm-wave instruments under development.
\end{abstract}

Key words: Dust: extinction, Cosmology: observations, Galaxies: evolution, Galaxies: formation, gravitational lensing, Radio continuum: galaxies

\section{Introduction}

Discovering the process by which the dense, gravitationally bound galaxies formed in the Universe from an initially almost uniform gas, and understand-

1 Corresponding author. E-mail: awb@astro.caltech.edu 
ing the way their constituent populations of stars were born is a key goal of modern physical cosmology. A wide range of well understood physical processes are involved; including general relativity, gas dynamics and cooling physics, nuclear reactions and radiative transfer. However, the range of possible initial conditions and the non-linear nature of most of the events, starting with the collapse of primordial density perturbations, ensure that these intimately connected processes can generate a very wide range of possible scenarios and outcomes. Galaxy formation can be studied by attempting to reproduce the observed Universe via analytical models and numerical simulations. The information required to constrain these models is provided by both forensic studies of the current constituents of the Universe, including stellar ages, chemical abundances and the sizes and shapes of galaxies, and by direct observations of the galaxy formation process taking place in the young Universe at great distances. Direct observations exploit both the light emitted by distant galaxies, and the signature of absorption due to intervening structures along the line of sight, and began almost 50 years ago using sensitive optical and radio telescopes. Astronomers must now use all available frequencies of radiation to probe the properties of the Universe, from the lowest energy radio waves to the highest-energy $\gamma$-rays. It is vital to combine the complementary information that can be determined about the constituents of the Universe at different wavelengths in order to make progress in our understanding.

This review discusses the results of a new type of direct observation of the galaxy formation process, made possible by the development of powerful new radiation detectors sensitive to wavelengths in the range $200 \mu \mathrm{m}$ to about $1 \mathrm{~mm}$ : the submillimeter (submm) waveband. The detection of submm radiation from distant galaxies is one of the most recent developments in observational cosmology, and has finally brought this region of the electromagnetic spectrum into use for making cosmological observations not directly connected with the cosmic microwave background (CMB; Partridge and Peebles, 1967). With the possible exception of the hardest X-ray wavebands, studies of distant galaxies in the submm waveband remained elusive for the longest period. We will also discuss some observations at the mid- and far-infrared(IR) wavebands that bound the submm waveband at short wavelengths, usually defined as the wavelength ranges from about 5-40 and 40-200 $\mu \mathrm{m}$, respectively.

The most significant reason for the late flowering of submm cosmology is the technical challenge of building sensitive receivers that work efficiently at the boundary between radio-type coherent and optical-like incoherent detection techniques. In addition, atmospheric emission and absorption permits sensitive submm observations from only high mountain sites, and only in specific atmospheric windows. The zenith opacity from the best sites in the clearest submm atmospheric window at $850 \mu \mathrm{m}$ is typically about 0.1 . Furthermore, the long wavelength of submm radiation limits spatial resolution unless very large filled or synthetic apertures are available. The largest single apertures 
available at present are in the $10-30 \mathrm{~m}$ class, providing spatial resolution of order 10 arcsec. This resolution is much coarser than the sub-arcsec resolution of optical and near-IR observations. The appearance of the same region of sky at optical and submm wavelengths is compared in Fig. 1 to illustrate this point: the multicolor optical image was obtained using the Hale 5-m telescope at Mt. Palomar, while the $850-\mu \mathrm{m}$ submm image was obtained using the 15-m James Clerk Maxwell Telescope (JCMT) on Mauna Kea. Interferometers can dramatically enhance the resolution of images, but so far have only operated at longer mm wavelengths. The commissioning of the 8-element Sub-Millimeter Array (SMA; Ho, 2000)[2 on Mauna Kea in Hawaii with baselines of up to about $500 \mathrm{~m}$, the first dedicated submm-wave interferometer, will provide images with subarcsecond resolution. The much larger 64-element Atacama Large Millimeter Array (ALMA; Wootten, 2001) ${ }^{3}$ will be in service at the end of decade.

A key development was the commissioning of the Submillimetre CommonUser Bolometer Array (SCUBA) camera at the JCMT in 1997 (Holland et al., 1999). SCUBA images the sky in the atmospheric windows at both 450 and $850 \mu \mathrm{m}$ in a 2.5-arcmin-wide field, using hexagonal close-packed arrays of 91 and 37 bolometer detectors at the respective wavelengths. SCUBA provided a dramatic leap forward from the pre-existing single-pixel or one-dimensional array instruments available. The combination of field of view and sensitivity was sufficient to enable the first searches for submm-wave emission from previously unknown distant galaxies. The Max-Planck Millimetre Bolometer Array (MAMBO; Kreysa et al., 1998) is a 1.25-mm camera with similar capabilities to SCUBA, which operates during the winter from the Institut de Radio Astronomie Millimétrique (IRAM) 30-m telescope on Pico Veleta in Spain. A similar device - the SEST Imaging Bolometer Array (SIMBA) - designed at Onsala in Sweden is soon to begin operation on the 15-m Swedish-ESO Submillimetre Telescope (SEST) in Chile, providing a sensitive submm imaging capability in the South. The capability of $\mathrm{mm}$ and submm-wave observatories is not standing still: a number of larger, more sensitive mm- and submmwave cameras are under construction, including the SHARC-II (Dowell et al., 2001), BOLOCAM (Glenn et al., 1998) and SCUBA-II instruments. 4 Bolometer technology continues to advance. The advent of extremely stable superconducting bolometers that require no bias current and can be read out using multiplexed cold electronics, should ultimately allow the construction of very large submm detector arrays of order $10^{4-5}$ elements (for example Benford et

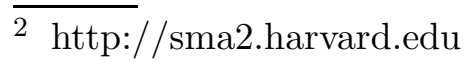

3 http://www.alma.nrao.edu

4 Details can be found in Table 3 . The next-generation SCUBA-II camera for the JCMT is under development at the United Kingdom Astronomy Technology Centre (UKATC). See http://www.jach.hawaii.edu/JACpublic/JCMT/Continuum observing/SCUBA-2/home.html. 


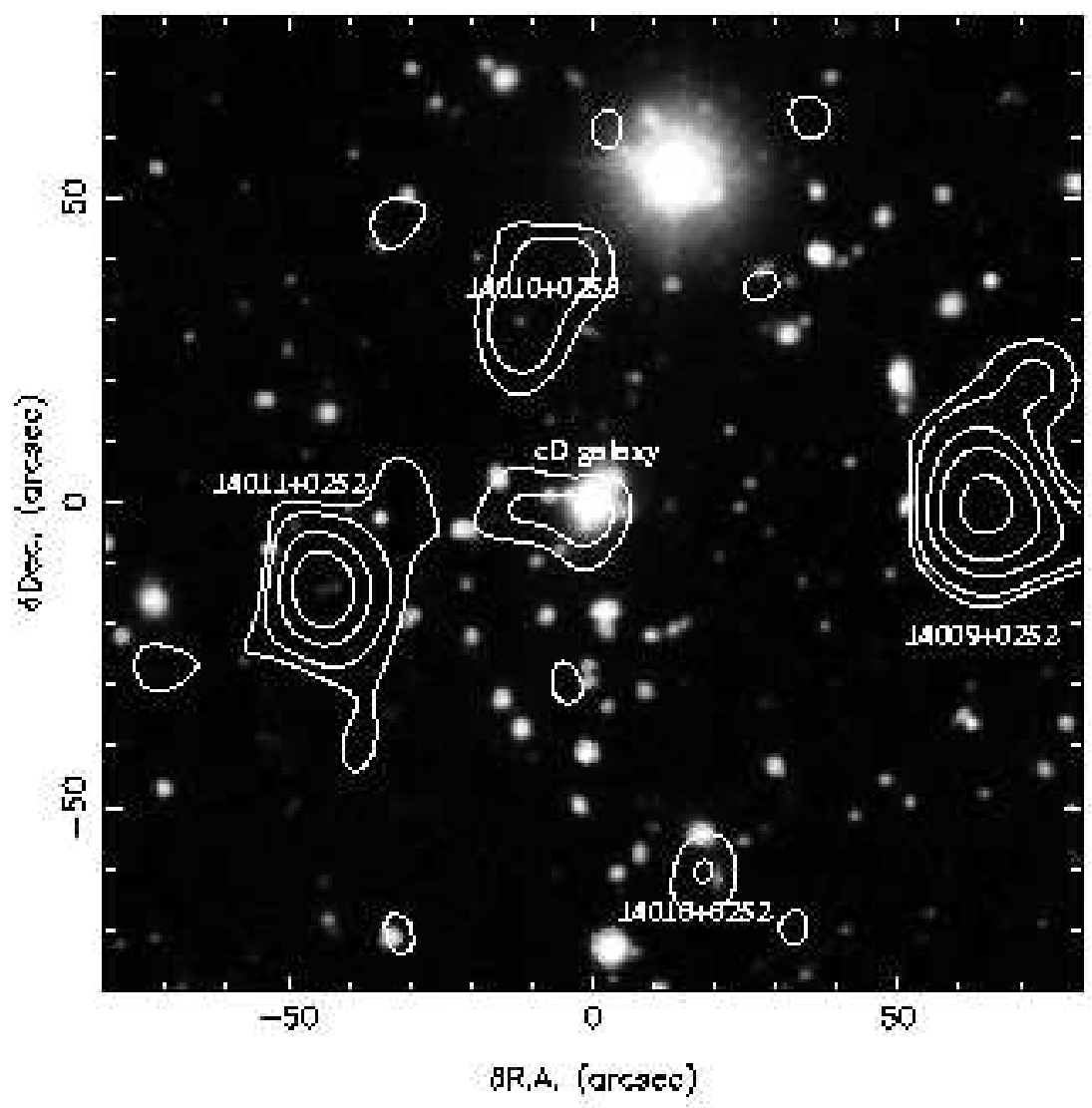

Fig. 1. A comparison of deep optical and submm views of the sky. The background image is a 3-color optical image of the rich cluster of galaxies Abell 1835 at the low/moderate redshift $z=0.25$ (Smail et al., 1998b) taken using the 5-m Hale telescope, overlaid with the 14-arcsec resolution contours of a SCUBA $850-\mu \mathrm{m}$ submm-wave image of the same field (Ivison et al., 2000a). North is up and East to the left. The brightest SCUBA galaxies at $(-45,-15),(65,0)$ and $(20,-60)$, and the central cD galaxy (Edge et al., 1999), all have clear radio detections at a frequency of $1.4 \mathrm{GHz}$ in images with higher spatial resolution than the SCUBA contours, obtained at the Very Large Array (VLA), supporting their reality. The bright SCUBA galaxy at $(-45,-15)$ is associated with SMM J14011+0253, an interacting pair of galaxies at redshift $z=2.56$ in the background of the cluster (Frayer et al., 1999). Spectacular fragmented structure appears in the Easterly red component of this galaxy in Hubble Space Telescope (HST) images (Fig. 18).

al., 2000). SCUBA-II is likely to be the first instrument to exploit this technology, providing a $8 \times 8$ - $\operatorname{arcmin}^{2}$ field of view at the resolution limit of the JCMT.

The first extragalactic submm/mm surveys using SCUBA and MAMBO revealed a population of very luminous high-redshift galaxies, which as a population, were responsible for the release of a significant fraction of the energy 
generated by all galaxies over the history of the Universe (Blain et al., 1999b). Almost 200 of these galaxies are now known (Smail et al., 1997; Barger et al., 1998; Hughes et al., 1998; Barger et al., 1999; Eales et al., 1999, 2000; Lilly et al., 1999; Bertoldi et al., 2000; Borys et al., 2002; Chapman et al., 2002a; Cowie et al., 2002; Dannerbauer et al., 2002; Fox et al., 2002; Scott et al., 2002; Smail et al., 2002; Webb et al., 2002a). There is strong evidence that almost all of these galaxies are at redshifts greater than unity, and that the median redshift of the population is likely to be of order 2-3 (Smail et al., 2000, 2002). However, only a handful of these objects have certain redshifts and well-determined properties at other wavelengths (Frayer et al., 1998, 1999; Ivison et al., 1998, 2001; Kneib et al., 2002). The results of these mm/submm surveys provide complementary information to deep surveys for galaxies made in the radio (Richards, 2000), far-IR (Puget et al., 1999), mid-IR (Elbaz et al., 1999) and optical (Steidel et al., 1999) wavebands. Submm observations are a vital component of the search for a coherent picture of the formation and evolution of galaxies, which draws on data from all wavebands where the distant Universe can be observed.

In this review, we describe the key features of the submm emission processes in galaxies. We summarize the current, developing state of submm-wave observations of distant galaxies, including the results of both blank-field surveys, and targeted observations of known high-redshift galaxies, including radio-galaxies, optically-selected quasars/QSOs, X-ray detected active galactic nuclei (AGNs) and optically-selected Lyman-break galaxies (LBGs). Submm-wave surveys are not immune to selection effects, and we discuss their strengths and weaknesses. We describe the properties of the class of submm-luminous galaxies, and discuss the key results that are required to make significant progress in understanding them. We consider the relationship between the submm-selected galaxies and other populations of high-redshift galaxies, and describe models that can account for the properties of submm-selected galaxies. We introduce the unusually significant effects of the magnification of distant submm-selected galaxies due to gravitational lensing (Schneider et al., 1992). Finally, we recap the key developments that are keenly awaited in the field, and describe some of the exciting science that will be possible in the next decade using future instruments.

The cosmological parameter values assumed are generally listed where they appear. We usually adopt a flat world model with a Hubble constant $H_{0}=$ $65 \mathrm{~km} \mathrm{~s}^{-1} \mathrm{Mpc}^{-1}$, a density parameter in matter $\Omega_{\mathrm{m}}=0.3$ and a cosmological constant $\Omega_{\Lambda}=0.7$. 


\section{Submm-wave emission from galaxies}

There are two major sources of submm radiation from galaxies: thermal continuum emission from dust grains, the solid phase of the interstellar medium (ISM), and line emission from atomic and molecular transitions in the interstellar gas. The ladder of carbon monoxide $(\mathrm{CO})$ rotational transitions, spaced every $115 \mathrm{GHz}$, is the most important source of molecular line emission, but there is a rich zoo of other emitting molecules in the denser phases of the ISM. Submm surveys for distant galaxies have so far been made using cameras that detect only continuum dust emission, and so this will be the main focus of this review. However, the search for line emission is already important, and its study will become increasingly significant. The spectral resolution provided by line observations reveals much more about the physical and chemical conditions in the ISM, for studies of kinematics, metallicity and excitation conditions. Molecular lines can also be used to obtain a very accurate spectroscopic redshift for the ISM in high-redshift galaxies with prior optical redshifts (for example Frayer et al., 1998). Searches for redshifts at $\mathrm{cm}$ and (sub)mm wavelengths using $\mathrm{CO}$ lines will be possible using future telescopes.

The best studied regions of the Universe in the submm waveband are Giant Molecular Clouds (GMCs) in the Milky Way, in which ongoing star formation is taking place (Hollenbach and Tielens, 1997). GMCs are perhaps very lowluminosity archetypes for distant dusty galaxies, although these galaxies have far-IR luminosities that are up to 4 orders of magnitude greater than that of the whole Milky Way.

Detailed, resolved submm-wave images and spectra only exist for low-redshift galaxies (for example Regan et al., 2001; Sakamoto et al., 1999), and it is often necessary to use them as templates to interpret the properties of more distant galaxies. A very important class of well-studied galaxies similar in luminosity, and perhaps in physical properties, to high-redshift submm galaxies are the ultraluminous IR galaxies (ULIRGs) discovered in the InfraRed Astronomy Satellite (IRAS) all-sky survey in the mid 1980's (see the review by Sanders and Mirabel, 1996). ULIRGs are usually defined as having a bolometric luminosity, integrated over all wavelengths at which dust emission dominates the SED (from about $1 \mathrm{~mm}-8 \mu \mathrm{m}$ ), in excess of $10^{12} \mathrm{~L}_{\odot}$. most luminous of all galaxies, but number less than $0.1 \%$ of galaxies in the local Universe. Due to their selection by $I R A S$, they are typically at relatively low redshifts, less than about 0.3. The first IRAS-detected high-redshift ULIRG was identified by Rowan-Robinson et al. (1991) at $z=2.3$. The current record redshift for a galaxy detected by IRAS is $z=3.9$ for APM 08279+5255 (Irwin et al., 1998). Both these galaxies appear to be extremely luminous; however,

$\overline{51 \mathrm{~L}_{\odot}}=3.84 \times 10^{26} \mathrm{~W}$ 


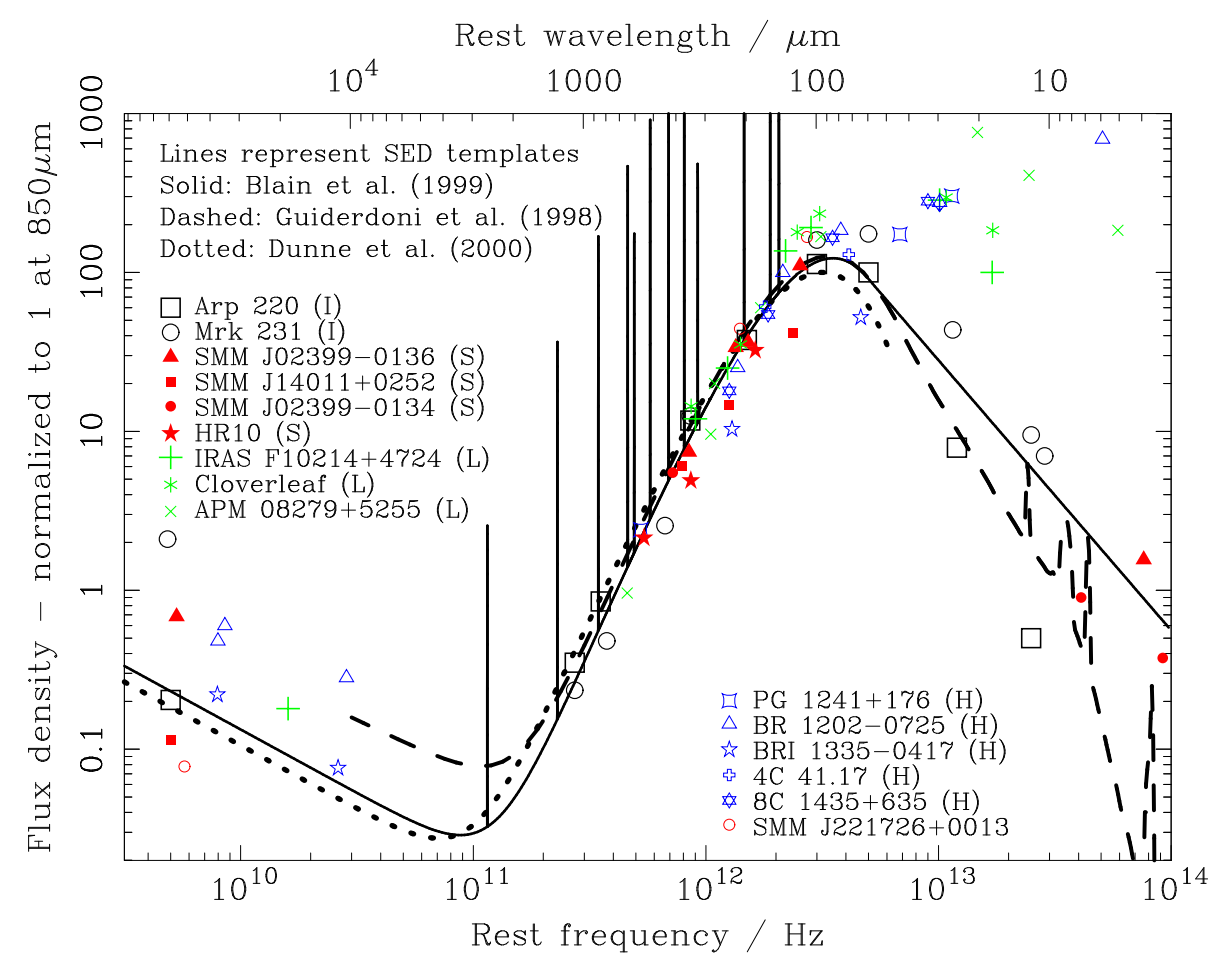

Fig. 2. Various observed restframe spectral energy distributions (SEDs) of galaxies from the radio to the near-IR wavebands. Two examples of the most luminous low-redshift galaxies detected by IRAS are included (I). Five very luminous high-redshift galaxies that have been, or could have been, detected directly in deep submm surveys (S), three high-redshift galaxies serendipitously magnified and made easier to study by the gravitational lensing effect of foreground galaxies and also detected by IRAS (L), and five high-redshift AGNs detected in optical or radio surveys $(\mathrm{H})$ are also shown. In addition, three template SEDs are shown. One includes the properties of $\mathrm{CO}$ and atomic fine-structure emission lines in the (sub)mm waveband at wavelengths from 100 to $3000 \mu \mathrm{m}$ (Blain et al., 2000b), one includes polycyclic aromatic hydrocarbon $(\mathrm{PAH})$ molecular emission features at wavelengths $\sim 10 \mu \mathrm{m}$ in the mid-IR waveband (Guiderdoni et al., 1998), and one is normalized to the typical SED of a sample of low-redshift IRAS galaxies (Dunne et al., 2000). For further information on far-IR SEDs see Dale et al. (2001). With the exception of the high-redshift AGNs and the lensed galaxies, the templates tend to provide a reasonable description of the SED at wavelengths around and longer than its peak, the regime probed by submm surveys. Less luminous galaxies like the Milky Way have dust spectra that peak at a wavelength about a factor of 2 longer than these templates (Reach et al., 1995).

their luminosities are boosted by at least a factor of ten due to gravitational lensing by foreground galaxies. A compilation of the properties of some of the most extreme ULIRGs is given by Rowan-Robinson (2000). The IR spectral energy distributions (SEDs) of some low-redshift ULIRGs and a compilation of results for the more sparsely sampled SEDs of high-redshift dusty galaxies are illustrated in Fig. 2. 


\subsection{The power source for dusty galaxies}

About $99 \%$ of the energy released by galaxies in the submm and far-IR wavebands is produced by thermal emission from dust grains; the remainder comes from fine-structure atomic and molecular rotational line emission. However, the source of the energy to power this emission by heating dust is often unclear. Any intense source of optical/ultraviolet (UV) radiation, either young high-mass stars or an accretion disk surrounding an AGN, would heat dust grains. Because dust emits a featureless modified blackbody spectrum, submm continuum observations can reveal little information about the physical conditions within the source. Regions of intense dust emission are very optically thick, and so little information can be obtained by observing optical or UV radiation.

In typical spiral galaxies, with relatively low far-IR luminosities of several $10^{10} \mathrm{~L}_{\odot}$ (for example Alton et al., 2000, 2001), the dust emission is known to be significantly extended, on the same scale as the 10-kpc stellar disk. 0 The emission is certainly associated with molecular gas rich star-forming regions distributed throughout the galaxy (Regan et al., 2001), in which dust is heated by the hot, young OB stars.

In intermediate luminosity galaxies, such as the interacting pair of spiral galaxies NGC 4038/4039 'the Antennae' (Mirabel et al., 1998; Wilson et al., 2000), the most intense knots of star-formation activity, from which most of the luminosity of the system emerges, are not coincident with either nucleus of the merging galaxies, but occur in a deeply dust-enshrouded overlap region of the ISM of the galaxies. This provides a strong argument that almost all of the energy in this system is being generated by star formation rather than an AGN.

In more luminous ULIRGs that are at sufficiently low redshift for their internal structure to be resolved, the great majority of the dust emission arises in a much smaller, sub-kpc region (Downes and Solomon, 1998; Sakamoto et al., 1999) within a merging system of galaxies. It is plausible that a significant fraction of the energy could be derived from an AGN surrounded by a very great column density of gas and dust that imposes many tens of magnitudes of extinction on the emission from the AGN in the optical and UV wavebands, and which remains optically thick even at near-IR wavelengths. Alternatively, an ongoing centrally condensed burst of star-formation activity, fueled by gas funneled into the center of the potential well of a pair of interacting galaxies by a bar instability (Mihos, 2000) is an equally plausible power source.

If the geometry of absorbing and scattering material is known or assumed,

$\overline{{ }^{6} 1 \mathrm{pc}}=3.09 \times 10^{16} \mathrm{~m}$ 
then radiative transfer models can be used to predict the SED of a galaxy, which should differ depending on whether the source of heating is a very small AGN with a very hard UV SED, or a more extended, softer-spectrum nuclear star-forming region (for example Granato et al., 1996). Note that the results are expected to be very sensitive to the assumed geometry (Witt et al., 1992). In merging galaxies this geometry is highly unlikely to be spherical or cylindrical, and is uncertain for the high-redshift galaxies of interest here. In the case of AGN heating, the SED would be is expected to peak at shorter wavelengths and the mid-IR SED would be expected to be flatter as compared with a more extended star-formation power source. Both these features would correspond to a greater fraction of hot dust expected in AGNs (see Fig. 2), and is seen clearly in the SEDs of low-redshift IRAS-detected QSOs (Sanders and Mirabel, 1996).

An alternative route to probing energy sources in these galaxies is provided by near- and mid-IR spectroscopy. At these longer wavelengths, the optical depth to the nucleus is less than in the optical/UV, and so the effects of the more intense, harder UV radiation field expected in the environs of an AGN can be observed directly. These include the excitation of characteristic highly-ionized lines, and the destruction of relatively fragile polycyclic aromatic hydrocarbon (PAH) molecules (Rigopoulou et al., 1999; Laurent et al., 2000; Tran et al., 2001), leading to the suppression of their distinctive emission and absorption features. Mid-IR spectroscopic observations with the successor to IRAS, the Infrared Space Observatory (ISO) in the mid 1990's indicated that most of the energy from low-redshift ULIRGs is likely generated by star-formation activity rather than AGN accretion. However, the fraction of ULIRGs containing AGN appears to increase at the highest luminosities (Sanders, 1999). This could be important at high redshifts, where the typical luminosity of dust-enshrouded galaxies is greater than in the local Universe. In addition, there may be dutycycle effects present to make an AGN accrete, and perhaps to be visible, for only a fraction of the duration of a ULIRG phase in the evolution of the galaxy (Kormendy and Sanders, 1988; Sanders et al., 1988; Archibald et al., 2002). X-ray observations also offer a way to investigate the power source, as all but the densest, most gas-rich galaxies, with particle column densities greater than $10^{24} \mathrm{~cm}^{-2}$ are transparent to hard $(>2 \mathrm{keV})$ X rays.

Ultra-high-resolution radio observations provide a route to probing the innermost regions of ULIRGs (Smith et al., 1998; Carilli and Taylor, 2000). By detected the diffuse emission and multiple point-like radio sources, expected from multiple supernova remnants, rather than a single point-like core and accompanying jet structures expected from an AGN, these observations suggest that high-mass star formation contributes at least a significant part of the luminosity of the ULIRGs Arp 220 and Mrk 273.

It is interesting to note that the observed correlation between the inferred mass 
of the black holes in the centers of galaxies and the stellar velocity dispersion of the surrounding galactic bulges, in which most of the stars in the Universe reside (Fukugita et al., 1999), might inform this discussion (Magorrian et al., 1998; Ferrarese and Merritt, 2000; Gebhardt et al., 2000). The mass of the bulge appears to exceed that of the black hole by a factor of about 200 . When hydrogen is processed in stellar nucleosynthesis, the mass-energy conversion efficiency is about $0.007 \epsilon_{*}$, where $\epsilon_{*}(\simeq 0.4)$ is the fraction of hydrogen burned in high-mass stars. When mass is accreted onto a black hole, the mass-energy conversion efficiency is expected to be about $0.1 \epsilon_{\mathrm{BH}}$, with $\epsilon_{\mathrm{BH}} \sim 1$ with the definition above. If accretion and nucleosynthesis were to generate the same amount of energy during the formation of a galaxy, then the ratio of mass contained in both processed stars and stellar remnants to that of a supermassive black hole is expected to be about $0.1 \epsilon_{\mathrm{BH}} / 0.007 \epsilon_{*}$. For $\epsilon_{*}=0.4$ and $\epsilon_{\mathrm{BH}}=1$, this ratio is about 36 . As a mass ratio of about 200 is observed, this implies that a greater amount of energy, by a factor of about 6 , is generated by high-mass star-formation activity than by gravitational accretion.

If the bulge-to-black-hole mass ratio is in fact greater than 200, then either the factor by which star formation dominates will exceed 6 , or the accretion must have been more than $10 \%$ efficient; that is $\epsilon_{\mathrm{BH}}>1$. If low-efficiency accretion dominates the process of the build up of mass in the central black hole, then less than 1 part in 7 of the luminosity generated during galaxy formation will be attributed to accretion as compared with high-mass star formation.

A greater amount of energy generated by star formation as compared with accretion processes appears to be favored by these circumstantial arguments.

\subsection{Continuum emission from dust}

The dust emission process is thermal, with dust grains emitting a modified blackbody spectrum. Grains of interstellar dust, distributed throughout the ISM of a galaxy, are heated to temperatures between about 20 and $200 \mathrm{~K}$, depending on the spectrum and intensity of the interstellar radiation field (ISRF), and the size and optical properties of the grains. Higher dust temperatures can be produced close to a powerful source of radiation, with dust subliming at temperatures of order $2000 \mathrm{~K}$. Very small grains can be heated far above their equilibrium temperatures by absorbing hard-UV photons (see Draine and $\mathrm{Li}, 2001)$. Lower dust temperatures, always exceeding the CMB temperature, are possible in opaque regions of the ISM that are shielded from intense heating, in the intergalactic medium or in regions with an intrinsically weak ISRF. Unless dust is heated by the ISRF in addition to the CMB the galaxy will not be detectable. We now consider the properties of the dust emission that are relevant to observations of high-redshift galaxies. 


\subsubsection{The emission spectrum, dust mass and temperature}

The minimum parameters necessary to describe the emission from dust grains are a temperature $T_{\mathrm{d}}$ and a form of the emissivity function $\epsilon_{\nu}$. In any galaxy there will be a distribution of dust temperatures, reflecting the different nature and environment of each grain. It is useful to use $T_{\mathrm{d}}$ to describe the coolest grains that contribute significantly to the energy output of a galaxy when discussing submm observations. In most cases, spatially and spectrally resolved images of galaxies are not available, and so it is reasonable to assume a volume-averaged description of the emissivity function as a function of frequency $\nu, \epsilon_{\nu} \propto \nu^{\beta}$. Values of $\beta$ in the range 1-2 are usually assumed. Scattering theory predicts that $\beta \rightarrow 2$ at low frequencies, while a value $\beta \simeq 1$ at high frequencies matches the general trend of the interstellar extinction curve that describes the properties of absorption of optical and UV radiation by the ISM (see Calzetti et al., 2000 and Section 2 of the review by Franceschini, 2002).

The simplest form of the emission spectrum/SED, $f_{\nu}$ is given by assuming that $f_{\nu} \propto \epsilon_{\nu} B_{\nu}$, in which $B_{\nu}$ is the Planck function $\left(2 k T_{\mathrm{d}} \nu^{2} / c^{2}\right.$ in the RayleighJeans limit, in units of $\mathrm{W} \mathrm{m}^{-2} \mathrm{~Hz}^{-1} \mathrm{sr}^{-1}$ ). This assumes that the emitting source is optically thin. For fitting spectra of galaxies found in deep submm surveys, we assume the simple $\epsilon_{\nu} B_{\nu}$ function to describe the SED. Dunne et al. (2000) and Dunne and Eales (2001) also use this functional form to fit the observed submm spectra of low-redshift galaxies. At the expense of adding another parameter to describe the SED, there is some physical motivation for a SED that includes an optical depth term,

$$
f_{\nu} \propto\left[1-\exp \left(-\tau_{\nu}\right)\right] B_{\nu}
$$

where $\tau_{\nu}$ is the frequency-dependent optical depth of the cloud, and is a multiple of $\epsilon_{\nu}$. This equation tends to the simpler $\epsilon_{\nu} B_{\nu}$ function at long wavelengths, and is assumed by, for example, Benford et al. (1999), Omont et al. (2001), Priddey and McMahon (2001) and Isaak et al. (2002), whose submm data for high-redshift AGNs tends to correspond to rest-frame frequencies that are relatively close to the peak of the SED. The extra parameter required to relate $\tau_{\nu}$ and $\epsilon_{\nu}$ can be defined as the frequency at which $\tau_{\nu}=1$ and the cloud becomes optically thick. If the opacity near a wavelength of $100 \mu \mathrm{m}$ is large, then the form of the peak of the SED tends to that of a blackbody spectrum. This suppresses the emission near to the SED peak relative to the emission in the Rayleigh-Jeans regime, and so this functional form provides a good fit to a set of submm and far-IR data with a higher value of $T_{\mathrm{d}}$ as compared with the $\epsilon_{\nu} B_{\nu}$ function, usually by about $10-20 \%$. However, because most observed SEDs for high-redshift galaxies have fewer than four data points (see Fig. 2), the difference is unlikely to be very significant. 
It is reasonable to assume that the mid-IR SED can be smoothly interpolated from a modified blackbody function at low frequencies to a power-law $f_{\nu} \propto \nu^{\alpha}$ in the mid-IR waveband on the high-frequency side of the spectral peak, in order to prevent the high-frequency SED from falling exponentially with a Wien spectrum. Hotter components of dust, emitting at shorter wavelengths, and ultimately stellar emission in the near-IR waveband, are certain to be present to reduce the steepness of the SED in the Wien regime. That an exponential Wien spectrum is inappropriate can be seen from the well-defined power-law mid-IR SEDs of Arp 220 and Mrk 231 shown in Fig. 2.

It is not always necessary to relate the SED $f_{\nu}$ and luminosity $L$ of a galaxy to the mass of dust $M_{\mathrm{d}}$ that it contains; this can of worms can remain closed by normalizing $f_{\nu}$ in a self-consistent way. However, if a dust mass is required, perhaps in order to estimate the metal content of the ISM, and so provide information about the integrated star-formation activity in the galaxy at earlier times (Hughes et al., 1997; Omont et al., 2001), then it is conventional to define a frequency-dependent mass-absorption coefficient $\kappa_{\nu}$ (Draine and Lee, 1984; with units of $\mathrm{m}^{2} \mathrm{~kg}^{-1}$ ), which is proportional to $\epsilon_{\nu} . \kappa_{\nu}$ is the 'effective area' for blackbody emission by a certain mass of dust,

$$
L \frac{f_{\nu}}{4 \pi \int f_{\nu}^{\prime} \mathrm{d} \nu^{\prime}}=\kappa_{\nu} B_{\nu} M_{\mathrm{d}}
$$

Values of $\kappa_{\nu}$ at a conventional frequency of around $1 \mathrm{~mm}$ are in the range $0.04-$ $0.15 \mathrm{~m}^{2} \mathrm{~kg}^{-1}$ (Hughes, 1996). Recent comparisons of optical extinction and submm emission from partially resolved edge-on spiral galaxies have tended to give values of $0.05-0.4 \mathrm{~m}^{2} \mathrm{~kg}^{-1}$ (see Fig. 4 of Alton et al., 2001). Domingue et al. (1999) derive $0.09 \mathrm{~m}^{2} \mathrm{~kg}^{-1}$ from similar far-IR, optical and submm data. Dunne et al. (2000) adopt a value of $0.077 \mathrm{~m}^{2} \mathrm{~kg}^{-1}$. Note that there is at least a factor of 3 uncertainty in these conversion factors.

An alternative dimensionless function $Q_{\nu}$ (Hildebrand, 1983) is sometimes used, which includes information about the mass/volume and surface area of a typical grain. If grains are assumed to be spherical (a big if), with bulk density $\rho$, radius $a$, and an emissive cross section $\pi a^{2}$, then $\kappa_{\nu}=3 Q_{\nu} / 4 a \rho$. $Q_{\nu} B_{\nu}$ is the effective emissivity function describing the energy flux from unit area of the dust grain surface. However, dust grains are more likely to be irregular in shape, possibly colloidal or in the form of whiskers. In that case, the emissivity per unit mass would be increased, and the dust mass associated with a fixed luminosity would be overestimated.

This geometrical uncertainty will inevitably result in uncertainty about the mass of dust. Hence, dust masses quoted in papers must be treated with caution, and may be best used as a comparative measure to distinguish galaxies. In general, we will avoid quoting dust masses, as this is unlikely to provide a 
reliable physical measure of the properties of galaxies until detailed resolved images are available, which is likely to require observations with the ALMA interferometer. This will be a recurring theme: observations with excellent sensitivity and spatial resolution using a large interferometer will resolve many of the questions raised throughout the paper.

Working from submm data, it is also difficult to assess the dust mass of a galaxy, even subject to the caveats above, without knowing its dust temperature. In the Rayleigh-Jeans spectral regime, the flux density from a galaxy $S_{\nu} \propto \nu^{2+\beta} M_{\mathrm{d}} T_{\mathrm{d}}$. If $T_{\mathrm{d}}$ is uncertain to within a factor, then $M_{\mathrm{d}}$ is uncertain to within the same factor. The dust mass is at least easier to estimate from a single long-wavelength observation than the luminosity $L$. As $L \propto M_{\mathrm{d}} T_{\mathrm{d}}^{4+\beta}$, or equivalently $L \propto S_{\nu} T_{\mathrm{d}}^{3+\beta}$, an uncertainty in $T_{\mathrm{d}}$ corresponds to a proportionally much larger uncertainty in the inferred value of $L$.

However, even if the dust mass can be determined reliably at low redshifts, it remains unclear whether the same procedure can be applied to determine the dust mass in more luminous and more distant systems. In order to determine the dust properties of high-redshift galaxies, data of the same quality that has been obtained for nearby galaxies is required. High-frequency submm/farIR observations are necessary to provide information about the rest-frame frequency of the peak of the SED for a high-redshift galaxy.

Given the current lack of resolved images of distant galaxies in the submm and far-IR wavebands, it is important to neither over-parametrize the descriptions nor overinterpret the results of observations of their SEDs. When spatiallyresolved, high-spectral resolution images are available, building on existing interferometric images of low-redshift dusty galaxies (Downes and Solomon, 1998; Sakamoto et al., 1999; Wilson et al., 2000), it should be possible to study the radiative transfer from sites of intense star formation and AGN in these geometrically complex opaque galaxies (see Ivison et al., 2000a, 2001). Models of the SEDs of dust-enshrouded AGN at different viewing angles have been developed by Granato et al. (1996), while star-forming regions embedded in a disk geometry have been analyzed by Devriendt et al. (1999). More powerful and efficient radiative-transfer codes are being developed (for example Abel et al., 1999), and it should be practical to develop detailed models of the appearance of galaxies with realistic geometries to account for future, highresolution multi-band submm images.

At present, we prefer to use a few simple parameters - $\alpha, \beta$ and $T_{\mathrm{d}}$ - to describe the essential features of the SEDs of dusty galaxies. Although such a model can encapsulate only a small part of the true complexity of the astrophysics in a galaxy, it can account for the existing SED data for a wide variety of dusty galaxies. A simple parametrization is preferable to a more baroque, and necessarily at present unconstrained, combination of geometry, dust mass 
and temperature. In the following section we list plausible values of our SED parameters and discuss the associated degeneracies in fitted values.

\subsection{The observed SEDs of dusty galaxies}

Information about the submm SEDs of galaxies has been gathered from targeted $\mathrm{mm}$ and submm observations of samples of low-redshift far-IR-selected galaxies from the IRAS catalog, (Andreani and Franceschini, 1996; Dunne et al., 2000; Lisenfeld et al., 2000; Dunne and Eales, 2001), and from far-IR and submm observations of high-redshift galaxies (see Fig. 2). The most extensive local survey (SLUGS; Dunne et al., 2000) consists of 850- $\mu \mathrm{m}$ SCUBA observations of 104 galaxies selected from the low-redshift IRAS Bright Galaxy Sample (BGS; Soifer et al., 1987). After fitting single-temperature $\epsilon_{\nu} B_{\nu}$ SEDs to the galaxies, Dunne et al. found that $\beta=1.3 \pm 0.2$ and $T_{\mathrm{d}}=38 \pm 3 \mathrm{~K}$ described the sample as a whole, with a natural dispersion in the properties from galaxy to galaxy. This IRAS-selected sample could be biased against less dusty galaxies. Dunne et al. are currently addressing this issue by observing a complementary sample of $B$-band selected low-redshift galaxies, which should be representative of optically luminous low-redshift galaxies as a whole.

Note, however, that when fitting only a few datapoints, there is a significant correlation between values of $\beta$ and $T_{\mathrm{d}}$ that can account for the data (left panel of Fig. 3). This can lead to ambiguity in the results, further emphasizing the difficulty in associating the dust mass or temperature inferred from a galaxy SED with the real physical properties of the galaxy.

The addition of $450-\mu \mathrm{m}$ data for 19 of the 104 galaxies in the SLUGS sample (Dunne and Eales, 2001), tends to split the galaxy SEDs into two categories: those that retain a definite $40-\mathrm{K}$ spectrum after including the $450-\mu \mathrm{m}$ data, and those for which cooler single-temperature SEDs, more similar to the SEDs of normal spiral galaxies, then provide a better fit. The first group are typically the more luminous galaxies in the sample, while the second includes 3 of the 5 lowest luminosity galaxies from the sample. Dunne and Eales (2001) propose a two-temperature model to account for the changes in light of the new 450$\mu \mathrm{m}$ data; however, a cooler single-temperature model with a larger value of $\beta$ provides a fit of similar quality. The results for one of the most significantly different fits is shown in the right-hand panel of Fig. 3. With the addition of the $450-\mu \mathrm{m}$ data, the nature of the SEDs of low-redshift, low-luminosity galaxies become more diverse. However, the more luminous galaxies, which are likely to be the most similar to typical high-redshift submm galaxies, are still described reasonably well by the original Dunne et al. (2000) 38-K SED.

An alternative approach is to determine an SED that can describe the ob- 

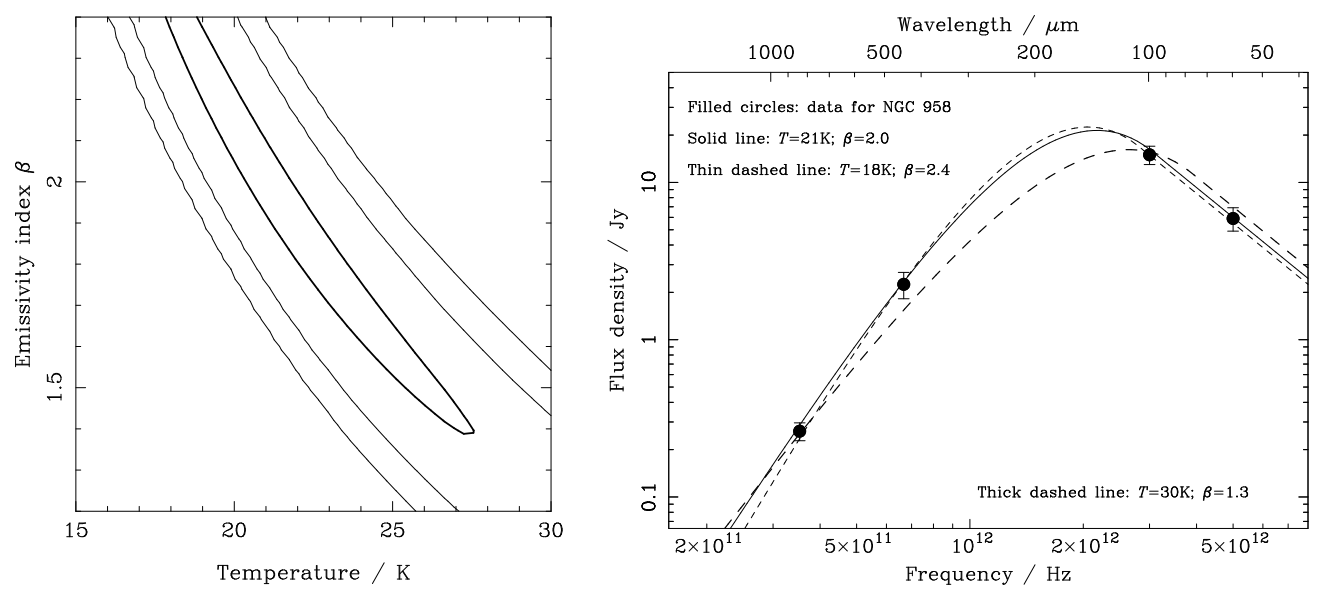

Fig. 3. An illustration of some of the issues involved in describing the SEDs of dusty galaxies. On the left is a probability contour plot that shows the $0.5,5 \times 10^{-3}$ and $5 \times 10^{-5}$ probability contours for a fit to an SED model defined by the variable parameters $\beta$ and $T_{\mathrm{d}}$ with a fixed value of $\alpha=-1.95$, taking into account four SED datapoints for the galaxy NGC 958 as shown in the right-hand panel (Dunne and Eales, 2001). Note that $1 \mathrm{Jy}=10^{-26} \mathrm{~W} \mathrm{~m}^{-2} \mathrm{~Hz}^{-1}$. Note that there is a very significant degeneracy in the fitted parameters. Adding additional data points with small errors close to the peak of the SED at $200 \mu \mathrm{m}$ reduces the extent of the probability contours by about $50 \%$, but they remain elongated in the same direction. Note that $\beta>2$ is not expected physically. On the right the data are compared with fitted single-temperature SEDs. The solid line is the best fit to the data. The dashed lines correspond to SEDs from the ends of the probability 'banana' shown in the left-hand panel. Note that without the $450-\mu \mathrm{m}$ point, the thick dashed curve describes the best-fit SED, which is defined by a significantly greater dust temperature. This SED is similar to that of a typical luminous IR galaxy, whereas the best fitting model with all four data points is much more like the SED of the Milky Way. Note that the shift in the best-fit model on adding $450-\mu \mathrm{m}$ data is generally less significant than in this case.

served flux density distribution of galaxies in the far-IR and submm wavebands, which are sensitive to galaxies at low, moderate and high redshifts (Blain et al., 1999b; Trentham et al., 1999; Barnard and Blain, 2002). Using the $\epsilon_{\nu} B_{\nu}$ functional form, values of $\beta \simeq 1.5$ and $T_{\mathrm{d}} \simeq 40 \mathrm{~K}$ are required to provide a good description of the data, rather similar to the values derived for temperatures of individual low-redshift luminous dusty galaxies in Dunne et al. (2000) and Lisenfeld et al. (2000), and for both the small number of high-redshift submm-selected galaxies with known redshifts and mid-IR spectral constraints (Ivison et al., 1998a, 2000a) and typical high-redshift QSOs (for example Benford et al., 1999). These temperatures are significantly less than those determined for the most extreme high-redshift galaxies (Lewis et al., 1998), and significantly greater than the $T_{\mathrm{d}}=17 \mathrm{~K}$ inferred from the maps of the Milky Way made using the all-sky survey from the FIRAS instrument on the Cosmic Background Explorer (COBE) satellite in the early 1990's (Reach et al., 1995). Note that there are examples of moderate-redshift 
infrared-selected galaxies with both hotter and colder typical dust temperatures than 40 K: see Deane and Trentham (2001) and Chapman et al. (2002d) respectively. At present it seems likely that a $40-\mathrm{K}$ dust temperature is a reasonable assumption for high-redshift submm-selected galaxies.

Inevitably, however, there will be a population of hotter high-redshift galaxies (Wilman et al., 2000; Trentham and Blain, 2001). These galaxies would be underrepresented in existing submm surveys, but may make a significant contribution to the $240-\mu \mathrm{m}$ background radiation intensity (Blain and Phillips, 2002). Further observational information to test the assumption of a 40-K dust temperature is keenly awaited. As we discuss below, in Section 2.6, the assumed dust temperature has a significant effect on the selection function of submm galaxy surveys, and on the properties that are inferred for the galaxies that are found in these surveys.

\subsection{Line emission}

Emission from molecular rotation and atomic fine-structure transition lines can be used to diagnose physical conditions within molecular clouds and photodissociation regions, and to trace out the velocity structure within. Some lines, such as those from $\mathrm{CS}, \mathrm{HCN}$ and $\mathrm{HCO}^{+}$are excited only in high-density gas, while others, including the most abundant polar species $\mathrm{CO}$, trace more typical regions in the ISM.

Studies of many emission lines from molecular cloud regions in nearby galaxies are possible using existing $\mathrm{mm}$ and submm-wave telescopes (Wilson et al., 2000; Helfer, 2000). However, for more distant galaxies only CO lines have so far been detected in significant numbers, almost exclusively from galaxies which have been subject to strong gravitational lensing by foreground galaxies (see the summary in Combes et al., 1999). These observations are useful for deriving physical conditions within the sources, especially if multiple lines are detected (as in the case of APM 08279+5255; Downes et al. 1999b). The improved capabilities of the forthcoming $\mathrm{mm} / \mathrm{submm}$ interferometer arrays SMA, upgrades to the IRAM Plateau du Bure interferometer (PdBI), and the Combined Array for Research in Millimeter-wave Astronomy (CARMA) $\square$ and and ultimately the dramatically increased sensitivity of ALMA, will make high-redshift lines much easier to observe over the next decade (Combes et al., 1999; Blain et al., 2000b).

One of the most important uses of CO-line observations of distant submm galaxies found in continuum surveys is their ability to confirm an identification absolutely, by tying together an optical and submm redshift at the position

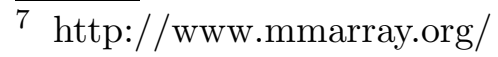


of the galaxy. So far this has been achieved for only three submm galaxies (Frayer et al., 1998, 1999; Kneib et al., 2002: see Figs. 14, 18 and 19). In principle, these observations could be made for all continuum-selected galaxies. The difficulty is the narrow fractional bandwidth available for receivers and correlators. Even at the relatively low frequency of $90 \mathrm{GHz}$, the redshift of the target must be known to better than $0.5 \%$ to ensure that a $300 \mathrm{~km} \mathrm{~s}^{-1}$ wide CO line, typical of a massive galaxy, with a width equivalent to $0.1 \%$ in redshift falls entirely within a $1-\mathrm{GHz}$ band. Future cm-, mm-, and submmwave instruments with wider bandwidths will significantly assist the the search for redshifts using molecular lines. Specially designed low-resolution, ultrawideband dispersive spectrometers covering many tens of $\mathrm{GHz}$ simultaneously on single-antenna mm-wave telescopes also promise to provide redshifts for submm galaxies (Glenn, 2001).

A complementary search for redshifted cm-wave $\mathrm{OH}$ megamaser emission to pinpoint the redshifts and positions of ultraluminous high-redshift galaxies could be possible using radio telescopes (Townsend et al., 2001). However, there are very stringent requirements on the acceptable level of radio frequency interference from terrestrial and satellite communications. Observations of low-redshift megamasers are described by Darling and Giovanelli (2001). Megamaser emission at high redshifts is discussed by Briggs (1999) in the context of the proposed Square Kilometer Array (SKA) meter/centimeterwave radio telescope. If it can operate at frequencies of several 10's of GHz, then the SKA is also likely to be an efficient detector of low-excitation highredshift CO lines (Carilli and Blain, 2002).

\subsubsection{Line emission contribution to continuum detections}

An interesting feature of the $\mathrm{CO}$ line emission from low-redshift galaxies is that lines can lie in the passbands of continuum instruments, and could contribute to the continuum flux inferred. For low-redshift galaxies, the $345-\mathrm{GHz}$ $\mathrm{CO}(3 \rightarrow 2)$ line lies within the $850-\mu \mathrm{m}$ atmospheric window, while the 691 $\mathrm{GHz} \mathrm{CO}(6 \rightarrow 5)$ and $230-\mathrm{GHz} \mathrm{CO}(2 \rightarrow 1)$ lines lie in the $450-\mu \mathrm{m}$ and $1.25-\mathrm{mm}$ windows, respectively.

Assuming a reasonable template spectrum (Blain et al., 2000b), the equivalent width in frequency of the $\mathrm{CO}(3 \rightarrow 2)$ line is $7.4 \mathrm{GHz}$. The passband of the current SCUBA $850-\mu \mathrm{m}(353-\mathrm{GHz})$ filter is about $120 \mu \mathrm{m}(50 \mathrm{GHz})$ wide, and so about $15 \%$ of the measured continuum flux density of a low-redshift galaxy in the $850-\mu \mathrm{m}$ channel is likely to be from the CO line. The high-frequency SCUBA passband in the $450-\mu \mathrm{m}$ atmospheric window is $75 \mathrm{GHz}$ wide, while the equivalent width of the $\mathrm{CO}(6 \rightarrow 5)$ transition is $3.3 \mathrm{GHz}$. Hence, a smaller $5 \%$ contribution to the continuum flux density from the line is expected at $450 \mu \mathrm{m}$. The $\mathrm{CO}(2 \rightarrow 1)$ line has an expected equivalent width of $9.2 \mathrm{GHz}$, 
while the wide MAMBO passband has half-power points at 210 and $290 \mathrm{GHz}$. Contamination of the flux densities detected by MAMBO by about $10 \%$ may thus be expected.

The largest of these correction factors is comparable to the calibration uncertainty in submm-wave observations, and could be relevant to the detailed interpretation of low-redshift observations. For example, the presence of the $\mathrm{CO}(3 \rightarrow 2)$ line in the $850-\mu \mathrm{m}$ window would shift the inferred continuum emissivity spectral index $\beta$ in the SLUGS survey from 1.3 to 1.52 . At high redshifts, any corrections are likely to be less significant, both because the relatively bright $\mathrm{CO}(3 \rightarrow 2)$ line redshifts out of the $850-\mu \mathrm{m}$ passband, and the equivalent width of lines in frequency space decreases as $(1+z)^{-1}$.

Although the contribution to measured submm-wave flux densities from line emission could be significant at the level of order $10 \%$, only a small fraction of the bolometric luminosity from galaxies is detected in the submm waveband. More than $99 \%$ of the bolometric luminosity still appears in the continuum, predominantly at shorter far-IR wavelengths.

\subsection{The observability of high-redshift dusty galaxies}

The detectable flux density at an observed frequency $\nu$ from a galaxy with bolometric luminosity $L$ at redshift $z$ with an intrinsic $\operatorname{SED} f_{\nu}$,

$$
S_{\nu}=\frac{1+z}{4 \pi D_{\mathrm{L}}^{2}} L \frac{f_{\nu(1+z)}}{\int f_{\nu^{\prime}} \mathrm{d} \nu^{\prime}}
$$

where $D_{\mathrm{L}}$ is the luminosity distance to redshift $z$ (for example Peebles, 1993).

The key feature that makes submm-wave observations of distant galaxies interesting is the ability to sample the SED of a target galaxy at wavelengths for which the SED is a strongly increasing function of frequency (Fig. 2). This ensures that distant galaxies are observed at a rest-frame wavelength closer to the peak of their SED. There is thus a strong, negative $\mathrm{K}$ correction, which leads to high-redshift galaxies being relatively easy to detect at submm wavelengths as compared with their low-redshift counterparts. This effect is illustrated in Fig. 4 for the template SED from Blain et al. (1999b) shown in Fig. 2. The strong K-correction effect applies at wavelengths longer than about $250 \mu \mathrm{m}$. At these wavelengths the flux density from galaxies at $z>1$ ceases to decline with the inverse square of distance, but instead remains approximately constant with increasing redshift. A window is thus opened to the detection of all galaxies with similar SEDs at redshifts up to $z \simeq 10-20$. The effect is more pronounced at longer wavelengths: in the mm waveband more distant galax- 


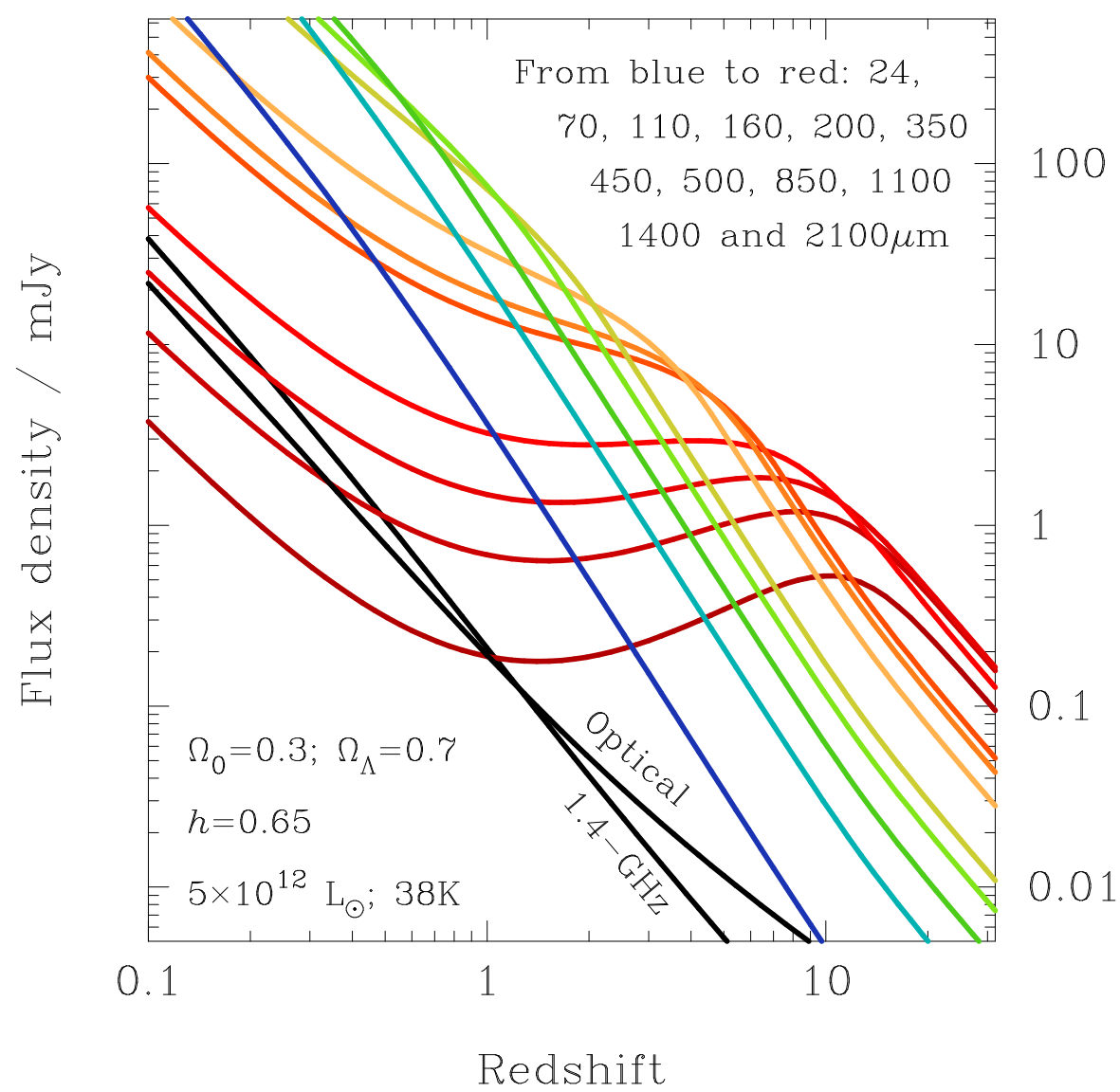

Fig. 4. The predicted flux density of a dusty galaxy as a function of redshift in various submm atmospheric windows, and at shorter wavelengths that will be probed by forthcoming space missions. Note the powerful $\mathrm{K}$ correction in the $\mathrm{mm}$ and submm wavebands at wavelengths longer than about $250 \mu \mathrm{m}$, which yields a flux density that is almost independent of redshift. The template spectrum is chosen to reproduce the typical properties of distant submm-selected galaxies (Fig. 2). Subtle effects due to the additional heating of dust by the CMB, and fine details of the radio SED of galaxies are not included; these effects are illustrated in Fig. 8.

ies are expected to produce greater flux densities than their more proximate counterparts.

Note that both the radio and optical flux-density-redshift relations decline steeply with increasing redshift, and so high-redshift galaxies are not selected preferentially in those wavebands. The advantage that faint radio and optical galaxy surveys have over submm surveys comes from the complementary probe of astrophysical signatures, and the combination of greater fields of view and finer angular resolution.

A submm telescope that is sufficiently sensitive to detect a certain class of galaxy at redshift $z \simeq 0.5$, can detect any similar galaxies out to a redshift $z \sim 10$ (Blain and Longair, 1993a). Note, however, that surveys to exploit this unusual K correction are not immune to selection effects. The K correction 
can also only be exploited at redshifts for which sufficient heavy elements are present in the ISM of the target galaxy to form enough dust to reprocess optical radiation. Nor does the K correction effect overcome cosmological surface brightness dimming for progressively more distant submm galaxies: the normal $(1+z)^{-4}$ reduction in surface brightness still applies; however, it is not expected to become significant until redshifts in excess of about 5 . Because submm-wave telescopes do not yet resolve distant galaxies, this effect cannot be observed at present. It may provide an opportunity to estimate redshifts for the most distant submm-selected galaxies when they can be resolved using ALMA.

\subsection{Submm-wave selection effects}

Deep submm-wave observations image the high-redshift Universe with very little contamination from low-redshift galaxies, and can potentially find a population of galaxies that is quite different to those detected in conventional deep optical surveys, and which could be undetectable in these surveys. The complementarity of submm and optical observations is illustrated by the very limited overlap between galaxies detected in the deep submm-optical image shown in Fig. 1. However, submm surveys are certainly subject to selection effects. In Fig. 5 the flux-density-redshift relation for a submm-luminous galaxy with a fixed bolometric luminosity is presented as a function of its SED parameters $-T_{\mathrm{d}}, \alpha$ and $\beta$. The relatively minor effects of different assumed cosmological models are also shown. Changing the dust temperature has the greatest effect. The inferred luminosity of a dusty galaxy for a fixed observed submm flux density goes up by a factor of 10 if the dust temperature is doubled, at all but the very highest redshifts. There is thus a significant potential bias in submm surveys against the detection of galaxies with hotter dust temperatures for a given bolometric luminosity.

This effect was noted by Eales et al. (1999), when investigating the evolution of galaxies in the context of the results of deep SCUBA surveys. They suggested that the submm galaxies may be cooler than the temperatures of about $60 \mathrm{~K}$ usually assumed, and so their significance as a population of strongly evolving high-redshift galaxies may have been overestimated.

As discussed in Section 2.3, a cooler dust temperature of $40 \mathrm{~K}$ is compatible with observations of the SEDs of individual submm galaxies with confirmed redshifts detected in submm surveys (Ivison et al., 1998a, 2000a) and with the results of targeted observations of luminous low-redshift IRAS galaxies and high-redshift QSOs. If this temperature is assumed, then the inferences about galaxy evolution made from the results of submm surveys (Blain et al., 1999b, c; Eales et al., 2000; Smail et al., 2002) should be reliable. However, until a 

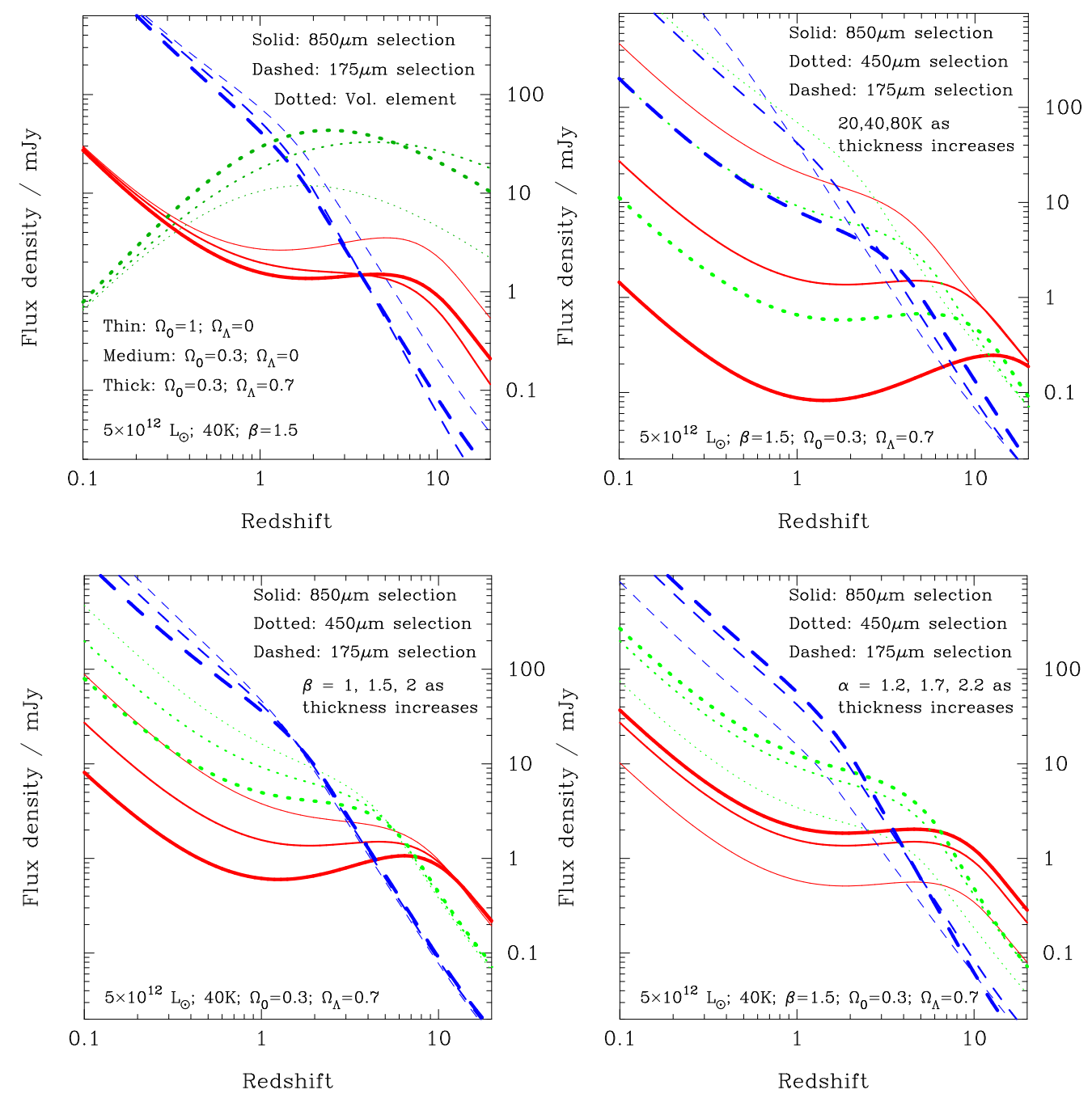

Fig. 5. Flux-density-redshift relations illustrating some of the uncertainties that apply to the interpretation of submm and far-IR surveys. In the top-left panel the relatively small effects of changing the world model parameters are shown. Note that changes to the volume element and flux density received counteract each other, and so the chosen cosmology has a small effect on the interpretation of surveys. The most significant effect - that of changing the dust temperature - is shown in the top-right panel. At $175 \mu \mathrm{m}$ for galaxies at moderate redshifts, the effect of temperature is rather small. However, at $850 \mu \mathrm{m}$, the effects are very significant, and must be remembered when interpreting the results of $850-\mu \mathrm{m}$ observations: doubling the dust temperature corresponds to increasing the luminosity associated with a given flux density by a factor of about 10 . The less significant effects of changing the dust emissivity index $\beta$ or the mid-IR spectral index $\alpha$ are shown in the bottom-left and bottom-right panels respectively.

large sample of submm galaxies with redshifts and multi-waveband SEDs is available, the possibility that a cold or hot population of high-redshift dusty galaxies could be missing from or misidentified in submm surveys cannot be ruled out (Eales et al., 1999; Blain and Phillips, 2002). The possible effects on inferred luminosities of different forms of the SED shown in Fig. 5 need 
to be taken seriously, especially when describing the properties of individual galaxies selected in submm surveys.

There is little reliable evidence for a systematic relationship between dust temperature and redshift. Observations of low-redshift IRAS galaxies (Andreani and Franceschini, 1996; Dunne et al., 2000), indicate that any variation of dust temperature with luminosity appears to be gradual. However, there is evidence for a significant and systematic change in the temperature of dusty galaxies with a wider range of luminosities, from about $20 \mathrm{~K}$ for low-redshift spirals (Reach et al., 1995; Alton et al., 2000; Dunne and Eales, 2001) to about $40 \mathrm{~K}$ for more luminous objects typical of the galaxies detected in the IRAS survey. Temperatures of up to $110 \mathrm{~K}$ are found for some extremely luminous high-redshift galaxies (Lewis et al., 1998).

We stress that there could be a significant selection effect in submm surveys that depends on the range of dust temperatures in the source population. The importance of such an effect can be quantified once a complete redshift distribution is available for a submm-selected galaxy sample.

\subsection{Deep submm-wave surveys}

Images of the redshift $z=0.25$ cluster of galaxies Abell 1835 in both the optical and submm wavebands were compared in Fig. 1. This image provides a realistic impression of the appearance of deep optical and submm images of the sky. Note that the only relatively low-redshift or cluster member galaxy that contributes any submm-wave flux is the central cD galaxy: the other cluster galaxies are either quiescent, neither forming stars nor heating dust, or are insufficiently luminous to be detectable at $850 \mu \mathrm{m}$ using SCUBA. Background galaxies at much greater redshift, which have faint optical counterparts as compared with the cluster member galaxies, dominate the image. This is a direct visual demonstration of the strong bias towards the detection of distant galaxies in submm-wave surveys that was illustrated in Fig. 4. These background galaxies are magnified by a factor of order 2-3 due to the gravitational lensing potential of the foreground cluster over the full extent of the image. The effects of gravitational lensing can be determined using accurate models of the cluster potential, that are constrained with the help of data from HST images and spectroscopic redshifts for multiply-imaged optically-selected galaxies. The uncertainty in the results is comparable to the uncertainty in the calibration of the submm images.

As shown in Fig. 1, existing deep submm images are much less visually stimulating than deep optical images, because their angular resolution is not sufficient to image the internal structure in distant galaxies. The limited resolution 
also imposes a confusion limit to the depth for submm surveys, at which the noise level is dominated not by atmospheric or instrumental noise but by the telescope resolution blurring together signals from faint unresolved galaxies. It takes about $50 \mathrm{~h}$ of integration using SCUBA to reach the practical confusion limit in a single field. Confusion is discussed in more detail in Section 3.1.

There are a variety of published results from deep submm galaxy surveys. Surveys aim to detect high-redshift galaxies exploiting the powerful K-correction effect in the submm waveband. Over $500 \mathrm{arcmin}^{2}$ of blank sky has been surveyed using SCUBA by several groups (Barger et al., 1998; Hughes et al., 1998; Barger et al., 1999a; Eales et al., 1999, 2000; Borys et al., 2002; Fox et al., 2002; Scott et al., 2002; Webb et al., 2002a). These range from an extremely deep survey in the area of the Hubble Deep Field-North (HDF-N; Williams et al. 1995) by Hughes et al. (1998) searching for the faintest detectable populations of submm galaxies, to wider-field shallower surveys to detect brighter sources that might be easier to follow-up and could be used to trace large-scale structure (Borys et al. 2002; Scott et al. 2002). About 30 5-arcmin ${ }^{2}$ lensed cluster fields have been imaged using SCUBA (Smail et al., 1997, 2002; Chapman et al., 2002a; Cowie et al., 2002; Kraiberg Knudsen et al., 2001; van der Werf and Kraiberg Knudsen, 2001), to various RMS depths between 0.5 and $8 \mathrm{mJy}$. 8 By exploiting the magnification effect of gravitational lensing, which extends over fields several arcminutes across, due to rich clusters of galaxies at moderate redshifts, the population of distant galaxies in the source plane behind the lensing cluster can be probed to greater depths than is possible in a blank field (Blain, 1998).

The detection rate of galaxies using SCUBA based on published papers appears to have declined over time since 1998. In significant part this is due to the absence of the sustained excellent observing conditions on Mauna Kea that were experienced during the El Nino winter of 1997-1998, just after SCUBA was commissioned.

A wide variety of complementary, and sometimes overlapping surveys have been made using MAMBO at the IRAM 30-m telescope (Bertoldi et al., 2000, 2001; Carilli et al., 2001), during several winters. These fields include the cluster Abell 2125 and the ESO-NTT Deep Field (Arnouts et al., 1999).

A compilation of the results from all the SCUBA and MAMBO surveys is presented in Figs. 9 and 10. A full summary of deep projects that have been undertaken or are underway can be found in Ivison (2001). In addition, larger shallower submm surveys of the Galaxy (Pierce-Price et al., 2000). 9 and per-

\footnotetext{
$81 \mathrm{Jy}=10^{-26} \mathrm{~W} \mathrm{~m}^{-2} \mathrm{~Hz}^{-1}$

9 A JCMT project (Vicki Barnard et al.) is currently searching for candidate highredshift galaxies detected in wide-field SCUBA images of star-forming regions in the Milky Way (Barnard et al., 2002).
} 
haps the CMB images obtained using the BOOMERANG balloon-borne experiment (Masi et al., 2001), can be used to search for brighter submm-wave galaxies.

\subsection{Submm observations of known high-redshift galaxies and QSOs}

The advent of SCUBA and MAMBO has also provided the opportunity to study the submm properties of large samples of interesting high-redshift galaxies, including almost all types of previously known distant galaxies. Isolated detections of high-redshift AGN-powered radio galaxies and QSOs were made in the mid 1990's using single-element bolometer detectors (for example Dunlop et al., 1994; Isaak et al., 1994); however, the compilation of statistical samples, and the secure rejection of contamination from fluctuating atmospheric noise have only been possible more recently, using SCUBA and MAMBO, and the 350- $\mu$ m one-dimensional bolometer array SHARC at the 10.4-m aperture Caltech Submillimeter Observatory (CSO) on Mauna Kea. A key advantage of observing these sources is that both their redshifts and some of their astrophysical properties are already known, in contrast with the submm-selected galaxies discovered in blank-field surveys. Some of the targeted galaxiesvery faint non-AGN radio galaxies, mid-IR-selected $I S O$ galaxies, and X-ray selected AGNs - have only been detected very recently. As the relationship between these populations of galaxies and submm-selected galaxies is still unclear, many of the limits will be discussed in the context of following up submm surveys in Section 4.

Targeted surveys include a search for submm-wave continuum emission from high-redshift AGN-powered radio galaxies (Archibald et al., 2001), and observations of various samples of optically-selected QSOs (for example Carilli et al., 2001; Isaak et al., 2002). In these observations a single bolometer is aimed at the position of the target. While this does not lead to a fully-sampled image of the sky, it provides a more rapid measurement of the flux density at a chosen position. The results have been the detection and characterization of the dust emission spectra for a range of luminous high-redshift galaxies and QSOs, including APM 08279+5255 (Lewis et al., 1998), the galaxy with the greatest apparent luminosity in the Universe. Barvainis and Ivison (2002) have targeted all the known galaxies magnified into multiple images by the gravitational lensing effect of foreground galaxies from the CASTLES gravitational lens imaging project, ${ }^{10}$ significantly expanding the list of high-redshift galaxies magnified by a foreground mass concentration with a submm detection. The SEDs of several of these galaxies are shown in Fig. 2.

Archibald et al. (2001) find evidence for significant evolution in the properties

\footnotetext{
$\overline{10}$ http://cfa-www.harvard.edu/castles.
} 
of dust emission with increasing redshift in a carefully selected sample of AGN radio galaxies, whose radio properties were chosen to be almost independent of the redshift of the observed galaxy. The results perhaps indicate that more intense star-formation activity, as traced by the submm emission, takes place alongside the radio source activity at higher redshifts, and so provide a possible clue to the formation and evolution of the massive elliptical galaxies thought to host radio galaxies. Hughes et al. (1997), Ivison et al. (1998b) and Omont et al. (2001) discuss the consequences of finding large masses of dust at high redshifts, in terms of the limited cosmic time available for the formation of the stars required to produce the metals and dust required to generate sufficiently intense submm emission from the host galaxy.

The radio galaxies detected by Archibald et al. (2001) in pointed singlebolometer SCUBA observations were followed up by imaging observations of the surrounding 5 -arcmin ${ }^{2}$ fields, to search for submm-loud companions. Ivison et al. (2000b) found that the surface density of submm galaxies in some of these fields is about an order of magnitude greater than that in a typical blank field, indicating a significant overdensity of sources. This is likely due to some radio galaxies being found in high-density regions of biased high-redshift galaxy formation, which are possibly 'protoclusters'-rich clusters of galaxies in the process of formation.

A similar targeted approach has been taken to try to detect submm-wave emission from optically-selected LBGs at redshifts between 2.5 and 4.5 (Steidel et al., 1999). The Lyman-break technique (Steidel et al., 1996) detects the restframe 91.2-nm neutral hydrogen absorption break in the SED of a galaxy as it passes through several broad-band filters. Large samples of candidate LBGs can be gathered using multi-color optical images from 4-m class telescopes. The efficiency of the selection method is of order $70 \%$ after spectroscopic confirmation of the candidates using 8/10-m class telescopes. The LBGs are the largest sample of spectroscopically confirmed high-redshift galaxies, with a well-defined luminosity function (Adelberger and Steidel, 2000), a surface density of order $10 \mathrm{arcmin}^{-2}$, and inferred star-formation rates between 1 and $10 \mathrm{M}_{\odot} \mathrm{yr}^{-1}$. They appear to be typical of the population of distant galaxies, and their spectra provide useful astrophysical information.

Observing the LBGs at submm wavelengths is an important goal, as an accurate determination of their submm-wave properties will investigate the link (if any) between the large well-studied LBG sample and the more enigmatic submm galaxy population (Blain et al., 1999c; Lilly et al., 1999; Adelberger and Steidel, 2000; Granato et al., 2001). At present, the typical very faint limits to the optical counterparts of the subset of submm galaxies with accurate positions (Smail et al., 1998a, 2002; Downes et al., 1999; Dannerbauer et al., 2002), and the detection of Extremely Red Object (ERO) galaxies (with $R-K>6$ ) as counterparts to a significant fraction of submm galaxies (Smail 
et al., 1999, 2002; Gear et al., 2000; Frayer et al., 2002; Ivison et al., 2001; Lutz et al., 2001), argue against a large overlap between the two populations. The direct submm detection of LBGs using SCUBA has been largely unsuccessful at the 0.5-mJy RMS level: a single galaxy out of 16 was detected by Chapman et al. (2000; 2002c), while Webb et al. (2002b) describe a low significance of overlap between LBGs and SCUBA galaxies in a wide-field survey. The LBG cB58 at $z=2.72$ (Ellingson et al., 1996; Frayer et al., 1997; Seitz et al., 1998; Pettini et al., 2000), which is magnified strongly (by a factor of 10-20) by a foreground cluster of galaxies at $z=0.37$, and is at least ten times brighter than a typical LBG, was detected in the $\mathrm{mm}$ and submm by Baker et al. (2001) and van der Werf et al. (2002). However, after correcting for lensing, its $850-\mu \mathrm{m}$ flux density is only about $0.1 \mathrm{mJy}$, below the level of confusion noise in SCUBA images, and similar to the flux density level of the statistical detection of high-redshift LBGs in the 850- $\mu \mathrm{m}$ SCUBA image of the HDF-N (Peacock et al., 2000). In the field surrounding an overdensity of LBGs at $z=3.09$, Chapman et al. (2001a) were successful in detecting bright submm emission that appears to be associated with diffuse sources of Lyman- $\alpha$ emission at the redshift of the overdensity, but were not included in the Lyman-break catalog. A key point to note is that the limits on submmwave emission from LBGs are typically lower than expected if the relationship between UV spectral slope and far-IR luminosity observed for low-redshift low-luminosity starburst galaxies (Meurer et al., 1999) continues to high redshifts. Goldader et al. (2002) indicate that the relationship does not appear to hold for the most luminous galaxies.

The required sensitivity for successful submm observations of typical LBGs seems to be deeper than can be achieved using existing instruments. Observations using future very sensitive, high-resolution interferometers certainly ALMA, and perhaps CARMA and SMA, will shed more light on the submmLBG connection.

The advent of the current generation of very sensitive X-ray observatories, Chandra and XMM-Newton, is generating a large sample of faint, hard X-ray sources, the luminosity of which is assumed to be dominated by high-redshift AGN (Fabian, 2000). Absorption and Compton scattering in large column densities of gas preferentially depletes soft X-rays, hardening the X-ray SEDs of gas-rich AGN. Such a population of hard, absorbed X-ray sources is required in order to account for the cosmic X-ray background radiation spectrum, which is harder than the typical SEDs of individual low-redshift AGN (Fabian and Barcons, 1992; Hasinger et al., 1996). Observations of the limited areas of the sky where both submm and X-ray data are available (Fabian et al., 1999; Hornschemeier et al., 2000; Mushotzky et al., 2001; Almaini et al., 2002) have tended to show little direct overlap between the X-ray and submm galaxies, although there are examples of X-ray-detected submm-wave galaxies (Bautz et al., 2000). The combined results of Bautz et al. and Fabian et al. reveal 
that 2 out of 9 SCUBA galaxies are detected by Chandra. In the larger-area brighter 8-mJy survey, Almaini et al. (2001) identify only 1 out of 17 SCUBA galaxies using Chandra. Page et al. (2002) discuss further the submm properties of X-ray sources. Perhaps of order $10 \%$ of known submm galaxies have faint hard X-ray counterparts that would be typical of dust-enshrouded AGN. There is also a statistical detection of excess submm-wave emission from the positions of faint high-redshift hard X-ray sources (Barger et al., 2001) and a positive submm-X-ray galaxy correlation function (Almaini et al., 2002). The lack of strong X-ray emission from a majority of submm galaxies lends circumstantial support to the idea that much of their luminosity is derived from star formation and not from AGN accretion. However, some submm galaxies may have hydrogen column densities, and thus optical depths to Compton scattering, that are sufficiently great to obscure soft X-ray radiation entirely $\left(>10^{24} \mathrm{~cm}^{-2}\right)$. Even if they contained powerful AGN, these submm galaxies would be very faint in Chandra surveys, which reach detection limits of order $10^{-17} \mathrm{erg} \mathrm{cm}^{-2} \mathrm{~s}^{-1}$ at soft $0.5-2 \mathrm{keV}$ wavelengths (Giacconi et al., 2002). They may be found in very deep observations using the the greater collecting area of $X M M-N e w t o n$ for hard X-ray photons. However, note that the 15 -arcsec resolution of $X M M$-Newton leads to confusion due to unresolved faint sources in the beam that is likely to impose a practical limit of order $10^{-15} \mathrm{erg} \mathrm{cm}^{-2} \mathrm{~s}^{-1}$ to the depth of a survey in the hard X-ray $2-8 \mathrm{keV}$ band (Barcons et al., 2002). Deconvolution of the images from joint XMM-Newton/Chandra deep fields, exploiting the sub-arcsec positional information from Chandra, will perhaps allow this limit to be exceeded.

Finally, galaxies detected in far-IR surveys using ISO (for example Puget et al., 1999) out to redshifts $z \sim 1$ have been targeted for SCUBA submm observations (Scott et al., 2000). The large arcmin-scale observing beam in $170-\mu \mathrm{m}$ $I S O$ surveys makes identification of submm counterparts difficult, but progress has been made by combining sub-arcsec resolution deep radio images. The results include some sources with apparently rather cool dust temperatures of order $30 \mathrm{~K}$ (Chapman et al., 2002d), and are generally consistent with redshifts less than unity and dust temperatures of less than about $50 \mathrm{~K}$ for most of the galaxies.

\subsection{Alternative strategy for deep submm surveys}

An alternative strategy for finding submm galaxies has also been tried: targeted submm observations of faint radio-selected galaxies with accurate positions and radio spectral index information detected at a 1.4-GHz flux density level brighter than 40- $\mu$ Jy using the VLA (for example Richards, 2000). The radio source population at these faint flux density levels is expected to include mostly high-redshift star-forming galaxies, and only a minority of sources with 
the more powerful synchrotron-emitting jets and lobes associated with particles accelerated by AGN. By selecting those faint radio sources with faint K-band counterparts (Barger et al., 2000; Chapman et al., 2001b), it should be possible to sift out low-redshift galaxies from the target sample, and so generate a concentrated sample of luminous high-redshift galaxies to study in the submm. From Fig. 4, it is clear that a high-redshift faint 20-100- $\mu$ Jy nonAGN 1.4-GHz radio source is likely to be a very luminous galaxy. Chapman et al. (2001b) claim that high-redshift submm galaxies can be detected using SCUBA at a rate of about one every hour using this method, which is significantly more rapid than the rate of about one every $10 \mathrm{~h}$ achieved in blank-field surveys. The efficiency of submm detection of optically-faint 1.4-GHz 20- $\mu \mathrm{Jy}$ radio galaxies at an $850-\mu \mathrm{m}$ flux density greater than about $5 \mathrm{mJy}$ is of order 30-40\%. Barger et al. (2000) detect 5/15 with $I>24$ at $6 \mathrm{mJy}$, and Chapman et al. (2001b) detect $20 / 47$ with $I>25$ at $4.5 \mathrm{mJy}$.

The difficulty comes in the interpretation of the results. The additional selection conditions of requiring first a radio detection, and then a faint optical counterpart will inevitably lead to the omission of a certain fraction of the submm galaxy population. Galaxies missing would include both the order of $15 \%$ of distant submm-selected galaxies that have relatively bright optical counterparts - the 'Class-2' SCUBA galaxies (Ivison et al., 2000a; Smail et al., 2002) - and any very high-redshift submm galaxies that cannot be detected at the VLA, despite lying on the far-IR-radio correlation (Condon, 1992; see Fig. 8). There is also likely to be a bias (at all redshifts) towards both detecting AGNs, in which radio flux densities are boosted above the level expected from the standard radio-far-IR correlation, and the exclusion of a small population of low-redshift submm galaxies, which would be too bright in the radio and $K$ band to be included in the survey. The rare, and perhaps especially interesting, submm galaxies at the lowest and highest redshifts are thus likely to be missing from radio pre-selected surveys, as are the distant submm galaxies with brighter optical magnitudes that are likely to be easiest to follow-up and investigate.

The size of these selection effects is difficult to quantify at present. However, their existence can be inferred from the diverse multiwaveband properties displayed by submm-selected galaxies from imaging surveys, a significant fraction of which are known not to be detected in deep radio observations (Downes et al., 1999; Smail et al., 2000). In some cases, the depth of the radio images used to make the comparison could be improved by a factor of several; it is possible, but we suspect unlikely, that all submm sources have radio counterparts lurking just below existing detection thresholds.

It is instructive to compare the results of the blank-field and faint radio preselected submm surveys. In a true 260-arcmin ${ }^{2}$ blank-field survey, Scott et al. (2002) found surface densities of $550_{-170}^{+100}$ and $180 \pm 60 \mathrm{deg}^{-2}$ submm galaxies 
brighter than 6 and 10 mJy respectively, while Borys et al. (2002) estimate $164 \pm 28 \mathrm{deg}^{-2}$ brighter than $12 \mathrm{mJy}$ in a 125 - $\operatorname{arcmin}^{2}$ survey, with a rather conservative error estimate from 12 detections. The surface densities resulting from the faint radio-selected investigations of Barger et al. (2000) and Chapman et al. (2001b) are $430 \mathrm{deg}^{-2}$ brighter than $4 \mathrm{mJy}$ and $135 \mathrm{deg}^{-2}$ brighter than $10 \mathrm{mJy} . \square$ This indicates incompleteness in the faint radio-selected counts by at least about $25 \%$. This is perfectly acceptable for gathering a list of highredshift galaxies for further study; however, for statistical analysis of a large sample of many tens of submm galaxies, the uncertainty introduced in the derived properties of the population due to incompleteness is expected to dominate the Poisson uncertainty, and so limit the accuracy of the inferences that can be made. Once the bright counts of distant $850-\mu \mathrm{m}$ galaxies are known from direct, hopefully unbiased, large-area SCUBA imaging surveys (Fox et al., 2002; Scott et al., 2002) and matched with very deep radio images (Ivison et al., 2002), then the value of this shortcut for compiling a large sample of submm galaxies can be assessed. The same strategy would be very valuable for wide-field MAMBO 1.2-mm surveys (Dannerbauer et al., 2002). At present, some care needs to be taken in the interpretation of faint radio pre-selected surveys.

\subsection{Determining redshifts of submm galaxies}

By virtue of their uniform selection function with redshift, it is impossible to even indicate the redshift of submm galaxies from single-wavelength submm flux densities alone. The relatively poor positional accuracy of submm images also makes it very difficult to identify an unambiguous optical counterpart for spectroscopic follow-up. This will be discussed in more detail when the properties of individual galaxies are described in Section 3. Here we discuss some general features of the prospects for determining their redshifts from submm, far-IR and radio observations.

\subsubsection{Photometric redshifts from far-IR SEDs}

The submm-far-IR SED of dusty galaxies is thermal, and so redshifting a fixed template SED affects the observed colors in exactly the same way as changing the dust temperature. This means that even when multi-frequency far-IR data is available, the redshift will be uncertain, unless there is information about the intrinsic dust temperature of the source. This effect is related

\footnotetext{
$\overline{11}$ Barger et al., (2000) also impose a limit of less than $840 \mathrm{deg}^{-2}$ brighter than $6 \mathrm{mJy}$ at $850-\mu \mathrm{m}$, based on serendipitous detections of sources in their 2.5-arcminwide SCUBA images around the AGN radio galaxies.
} 
to the selection effect in favor of cold galaxies illustrated in Fig. 5. The expected colors of dusty galaxies, as a function of $(1+z) / T_{\mathrm{d}}$, the parameter that can be constrained in light of this degeneracy, is shown in Fig. 6. Colors in a variety of observing bands in both submm atmospheric windows and for the observing bands of the MIPS instrument on the Space InfraRed Telescope Facility (SIRTF) ${ }^{12}$ satellite are included. It is vital to stress that without knowledge of the dust temperature, it is impossible to determine a redshift from any combination of multicolor broadband far-IR/submm data. The degeneracy between $T_{\mathrm{d}}$ and $z$ is lifted partially by combining information derived from radio observations, if $T_{\mathrm{d}}>60 \mathrm{~K}$. It may also become clear that there is a Universal temperature-luminosity relation that extends to high redshifts, and can be exploited to determine redshift information using a far-IR-submm color-magnitude relation. In the absence of such a Universal relation, far-IR and submm colors can only be used to fix the parameter $(1+z) / T_{\mathrm{d}}$ reliably.

\subsubsection{Radio-submm photometric redshifts}

In Fig. 4, an estimate of the flux density of radio emission of a template dusty galaxy was shown as a function of redshift. We now investigate the radio properties of submm galaxies, which provide useful information about their SED and redshift. There is an excellent observed correlation between the radio and far-IR (60- and 100- $\mu \mathrm{m}$ ) flux densities of low-redshift galaxies over 4 orders of magnitude in luminosity, reviewed by Condon (1992) and recently investigated out to $z \simeq 0.3$ by Yun et al. (2001). If this correlation is assumed to hold to high redshifts, then submm-selected galaxies should be detectable in the deepest $10-\mu \mathrm{Jy}-\mathrm{RMS} 1.4-\mathrm{GHz}$ VLA radio images out to redshifts of order 3. Note that as a result of this correlation, optical spectroscopy of faint nonAGN radio galaxies alone can be used to trace the evolution of star-formation activity to redshifts $z \simeq 1.2$, beyond which spectroscopic redshifts are hard to determine (Haarsma et al., 2000).

The far-IR-radio correlation is thought to be due to a match between the rate at which the optical/UV radiation from young stars is absorbed by dust on a local scale in star-forming regions of galaxies, and re-emitted as thermal far-IR radiation, and the radio luminosity from the same regions (Harwit and Pacini, 1975). The radio luminosity is due to both free-free emission in HiI regions, and more importantly at frequencies less than about $10 \mathrm{GHz}$, to the synchrotron emission from relativistic electrons accelerated in supernova shocks. If an AGN is present, then it is likely that its accretion disk will provide an additional source of UV photons to heat dust, and both the disk and outflows will generate shocks to accelerate relativistic electrons. There is little reason to expect these effects to be proportional, unlike UV heating by

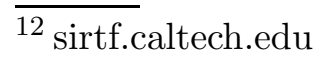




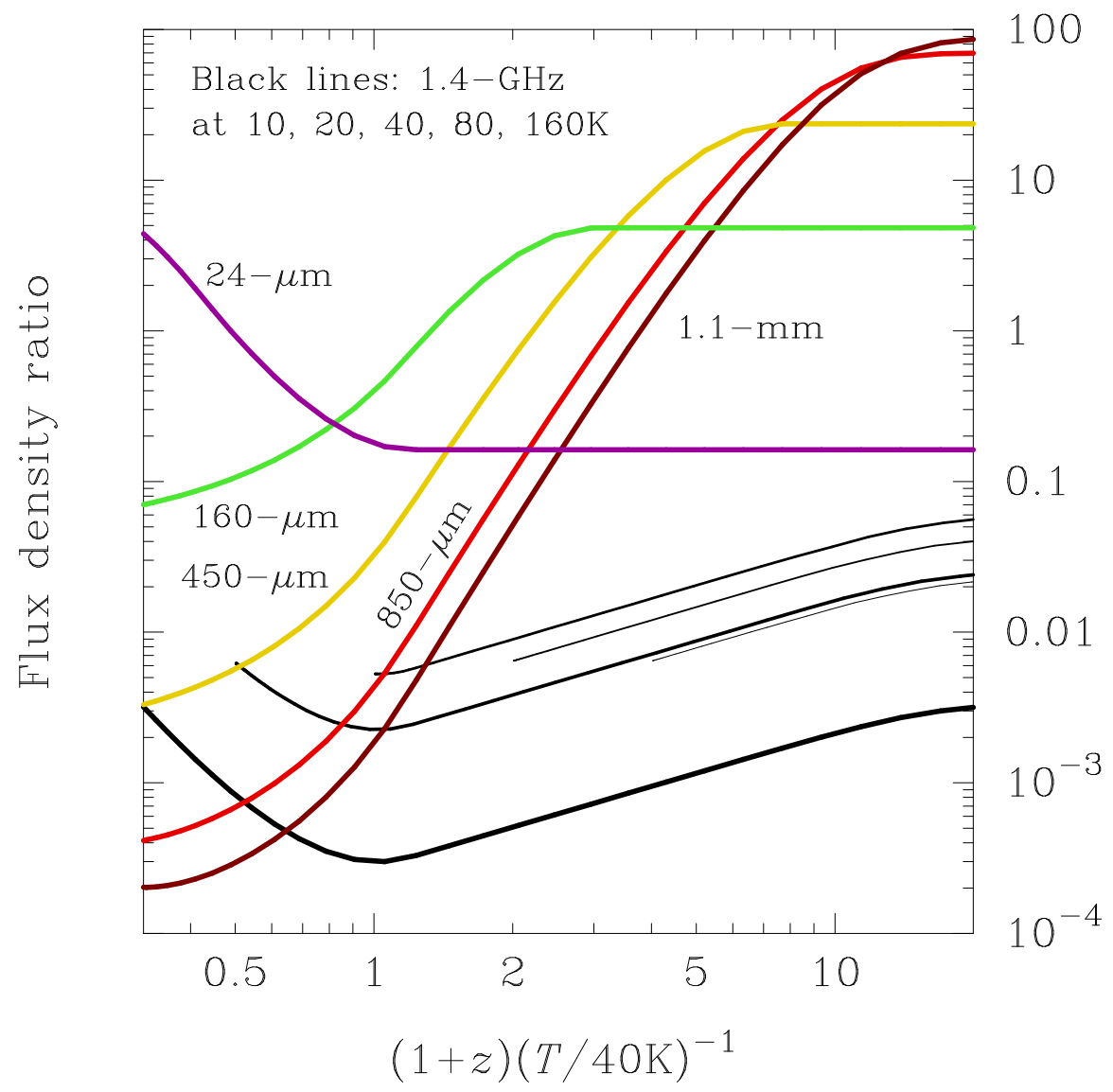

Fig. 6. The ratio of flux densities expected in different observing bands as a function of the degenerate redshift/dust temperature parameter, compared with the flux density expected in the $70-\mu \mathrm{m}$ band at which the SIRTF satellite will be very sensitive. Where the lines have steep gradients, measured colors from multi-band data locate the peak of the dust SED accurately in the observer's frame, providing a measurement of temperature-redshift. The degeneracy between $T_{\mathrm{d}}$ and $z$ can be lifted slightly by including radio data (Blain, 1999a; Yun and Carilli, 2002), if the dust temperature is greater than about $60 \mathrm{~K}$ (see also Fig. 7). If deep near-IR and optical images can be included, and the optical counterpart to the galaxy can be readily identified, then conventional photometric redshifts can be determined from stellar synthesis models. However, care must be taken as it is unclear whether the SEDs of very dusty galaxies have familiar restframe-optical spectral breaks.

massive stars and particle acceleration by supernova shocks in star-forming regions. Radio-quiet QSOs tend to lie on the radio-loud side of the far-IR-radio correlation, while radio-loud AGN can lie up to three orders of magnitude away.

Carilli and Yun $(1999,2000)$ demonstrated that the radio-submm color is a useful redshift indicator, assuming that dusty galaxies have simple synchrotron SEDs in the radio waveband and thermal dust spectra in the submm and far-IR wavebands. The radio-submm color was also considered in the interpretation of the redshifts of galaxies detected in the submm surveys by Hughes et al. 

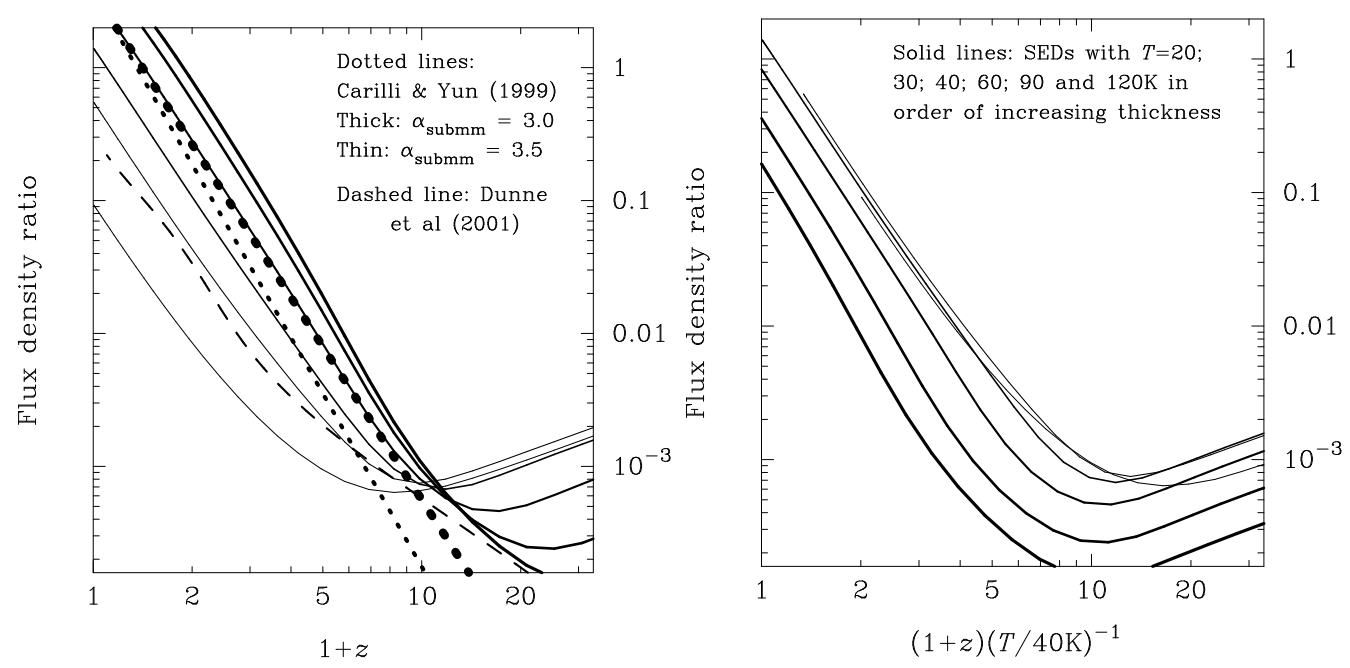

Fig. 7. The behavior of the Carilli-Yun $1.4-\mathrm{GHz}$ to $850-\mu \mathrm{m}$ radio-submm redshift indicator. The left panel shows the ratios of 1.4-GHz:850- $\mu \mathrm{m}$ flux density predicted from empirical SEDs by Carilli and Yun (1999; dotted lines) and Dunne et al. (2000; dashed line; see also Carilli and Yun, 2000). Predictions for the ratio based on the results of Blain (1999a) are also shown assuming radio-far-IR SEDs with various dust temperatures, but which all lie on the far-IR-radio correlation (Yun et al., 2001; solid lines). The flux ratio is a good indicator of redshift, clearly separating high- and low-redshift galaxies. Both synchrotron and free-free radio emission are included, and the dust temperature and radio properties evolve with redshift self-consistently, as modified by the CMB. In the right panel, the solid curves are replotted as a combined function of temperature and redshift, emphasizing that for $T_{\mathrm{d}}<60 \mathrm{~K}$, the inferred temperature and redshift are degenerate, just as for a thermal spectrum (Fig. 6). For $T_{\mathrm{d}}>60 \mathrm{~K}$ the flux ratio becomes a non-degenerate redshift indicator.

(1998), Lilly et al. (1999) and Eales et al. (2000). The Carilli-Yun redshift indicator is subject to a degeneracy between dust temperature and redshift, at dust temperatures less than about $60 \mathrm{~K}$ : see Fig. 7 in which its form is shown for a variety of SEDs. Hot, distant galaxies are difficult to distinguish from cool, low-redshift ones (Blain, 1999a, b). Despite this degeneracy, the CarilliYun redshift indicator is very useful, especially for investigating optically faint submm galaxies for which almost no other information is available (Smail et al., 2000). AGN, which are expected to be radio-loud as compared with the standard far-IR-radio correlation, lead to conservatively low Carilli-Yun estimated redshifts.

At the highest redshifts, two additional factors arise to modify the relation expected. First, synchrotron emission from relativistic electrons is likely to be suppressed. The intensity of synchrotron emission depends on only the energy density in the interstellar magnetic field, while the total cooling rate of the electrons depends on the sum of the energy densities in the interstellar magnetic field, for synchrotron losses, and in the ISRF, for inverse Compton scattering losses. The tight low-redshift far-IR-radio correlation implies that these energy densities are proportional over a very wide range of galaxy prop- 
erties (Völk, 1988). However, above some critical redshift, the energy density in the CMB will always rise to dominate the ISRF, upsetting this balance. Inverse-Compton electron cooling will then dominate and the amount of synchrotron emission will decline. Note, however, that free-free emission does not suffer this suppression at high redshifts, and so should remain detectable out to any practical redshift. The almost flat SED of free-free emission ensures a more favorable $\mathrm{K}$ correction for high-redshift galaxies than expected for a pure synchrotron emission spectrum. The free-free emission spectrum cuts off only at photon energies greater than the thermal energy of emitting electrons in HiI regions. In the absence of free-free optical depth effects, for $10^{4-5} \mathrm{~K}$ this corresponds to optical frequencies (Yun and Carilli, 2002). In Fig. 8 we show the effects of CMB suppression of synchrotron emission, for a ratio of energy densities in the magnetic field and the ISRF of 0.33 , which is reasonable for M82 and the Milky Way (Hummell, 1986). We assume a galaxy SED template that lies on the standard far-IR-radio correlation at $z=0$ (Condon, 1992).

Secondly, again most significant at high redshifts, a minimum dust temperature is imposed by the rising CMB temperature: dust must be hotter than the CMB. Given that observed dust temperatures in ultraluminous galaxies seem to lie in the range $40-100 \mathrm{~K}$, then this may become an important factor at redshifts $10<z<30$, if an early generation of stars generates the heavy elements required to form dust prior to these redshifts. For cooler Milky-Way-like SEDs, this effect would be important at $z \simeq 5$. An increase in dust temperature due to CMB heating at high redshifts shifts the peak of the dust SED to higher frequencies, counteracting the beneficial $\mathrm{K}$ correction illustrated in Fig. 4. As a result, there is a firm upper limit to the redshift at which submm continuum radiation can be exploited to image the most distant galaxies efficiently, even if these galaxies do contain dust. This is illustrated by the flux density-redshift relations at 230, 90 and $30 \mathrm{GHz}$ shown in Fig. 8: at redshifts greater than about 15 all three curves have the same form.

Both of these factors were included in the derivation of the curves in Fig. 7, which should thus provide an accurate guide to the usefulness of the CarilliYun redshift indicator out to the highest redshifts. Note that the indicator becomes of little use for the most distant galaxies, for which an almost constant radio:submm flux ratio of about $10^{-3}$ is expected. In addition, the hottest dusty galaxies may be more likely to contain AGN, and thus to lie on the radio-loud side of the far-IR-radio correlation. This effect could make the interpretation of Fig. 7 for determining redshifts ambiguous, even if $T_{\mathrm{d}}>60 \mathrm{~K}$. However, it should always guarantee a conservative estimate of the redshift for any observed galaxy.

Foreground absorption is not a problem for very high-redshift sources of any radio, submm or far-IR radiation. It is thus likely that the most sensitive future instruments at submm and radio wavelengths, ALMA and the SKA 


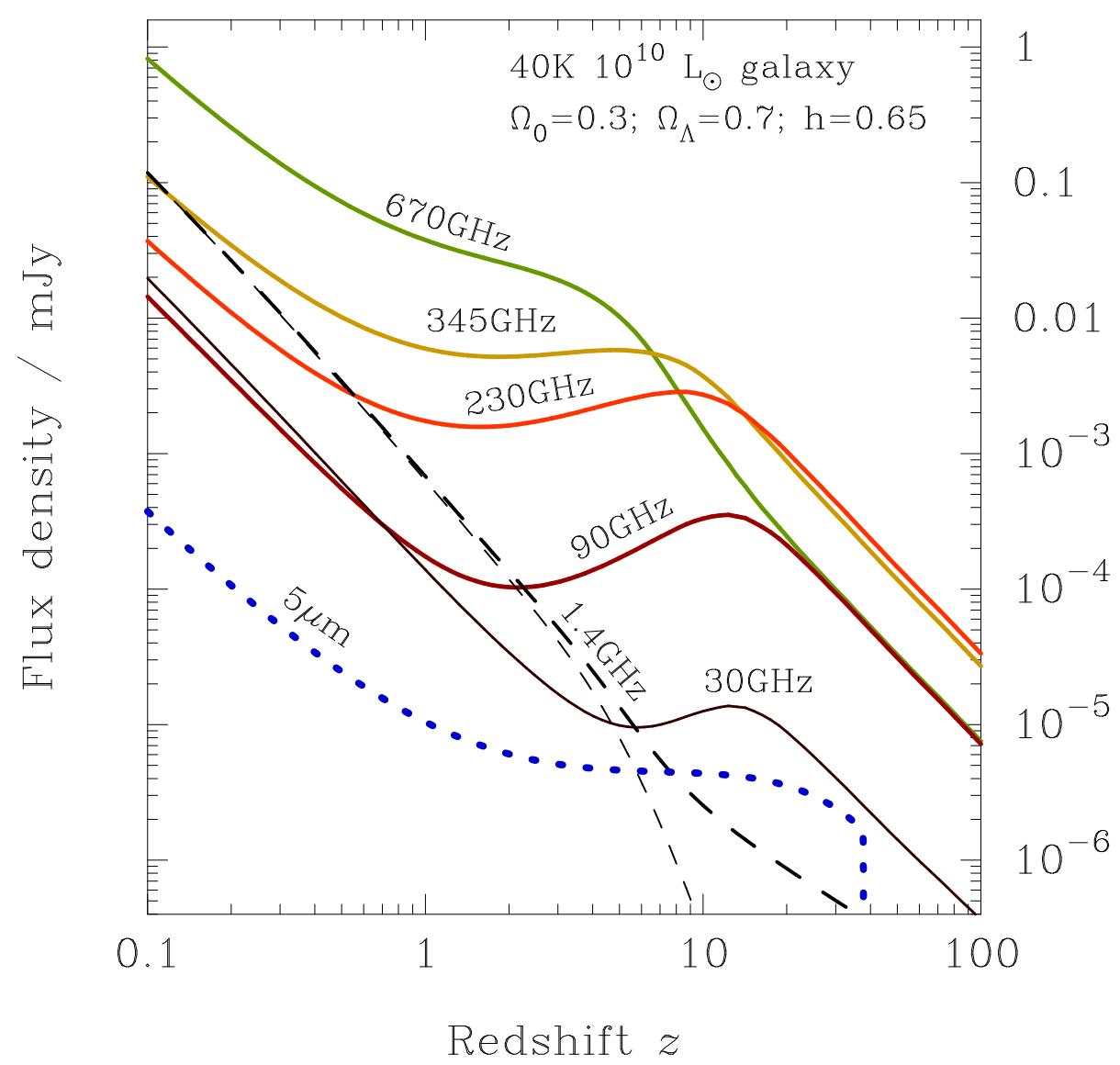

Fig. 8. Some key features of flux density-redshift relations expected at a range of wavelengths, extending to very high redshifts. CMB heating of dust at $z>10$ prevents the mm-wave $\mathrm{K}$ correction from assisting the detection of very high redshift galaxies: the flux density-redshift relation has the same redshift dependence beyond $z \simeq 15$ at 230,90 and $30 \mathrm{GHz}$. CMB cooling of relativistic electrons suppresses synchrotron radio emission beyond $z \sim 5$, as shown by the thin dashed line. Realistic free-free emission is also included in the model represented by the thick dashed line (Condon, 1992; Yun et al., 2001), significantly increasing the radio emission expected from very high redshifts. An estimate of the flux density from a $3 \times 10^{4}-\mathrm{K}$ stellar photosphere at $5 \mu \mathrm{m}$ is also shown, cutoff at the redshift beyond which Lyman- $\alpha$ absorption is redshifted through the band. Note that there is probably a maximum redshift above which dust does not exist, and so beyond which thermal emission from dust can never be detected; this effect is not included here.

will both be able to detect 'first light' galaxies. Note, however, that a mask of foreground structure may be significant for radio observations (Waxman and Loeb, 2000). A practical limit to the capability of determining the history of very early star formation from an 'SKA deep field' could also be set by the low, cosmologically-dimmed surface brightness of galaxies at the highest redshifts, and their potentially overlapping emission regions. The importance of both of these factors is expected to depend critically on the unknown physical sizes of the first galaxies. ALMA will probably be limited to observe galaxies out to a maximum redshift set by the requirement that sufficient metals have 
been generated to form obscuring dust at that early epoch. To compare what might be possible in the near-IR waveband, the emission from a $3 \times 10^{4}-\mathrm{K}$ blackbody stellar photosphere at $5 \mu \mathrm{m}$ is shown in Fig. 8, cutoff at the redshift beyond which absorption by redshifted Lyman- $\alpha$ becomes important in the band. This indicates the potential for probing the earliest galaxies using a near-IR camera on the Next Generation Space Telescope (NGST). Free-free emission and redshifted near-IR stellar emission may thus be the best routes to the detection of the first galaxies at redshifts greater than 10, if they exist.

\section{The observed properties of submm-selected galaxies}

Well over 100 submm-selected galaxies are now known (see Fig. 9), although their redshifts and detailed astrophysical properties are very largely uncertain. The key information available about their properties comes from observations of discrete galaxies made using the SCUBA and MAMBO bolometer array cameras at wavelengths of 450, 850 and $1200 \mu \mathrm{m}$. Counts of distant galaxies at far-IR wavelengths of 95 and $175 \mu \mathrm{m}$ have also been measured using the PHOT instrument aboard ISO. Limits to the counts at $2.8 \mathrm{~mm}$ have been obtained using the Berkeley-Illinois-Maryland Association (BIMA) mm-wave interferometer. The results of all the relevant observations are compiled in Figs. 9 and 10. Information is also available about the population of mid-IR 15- $\mu \mathrm{m}$ sources using the CAM instrument aboard ISO (Altieri et al., 1999; Elbaz et al., 1999): see Fig. 22.

Measurements of the observed intensity of background radiation from the radio to far-UV wavebands are shown in Fig. 11. The background measurements include lower limits obtained by summing over the observed counts plotted in Figs. 9 and 10. the deepest counts at 850 and $15 \mu \mathrm{m}$ come from surveys made in fields magnified by gravitational lensing clusters. Surface brightness conservation in the lensing process ensures that the mean background intensity in the direction of a lens should be the same as that in a blank field.

From the properties of the counts and backgrounds alone, without any details of the individual galaxies involved, it is possible to infer important details about the population of distant dust-enshrouded galaxies.

The significant surface density of the faint SCUBA and MAMBO galaxies, when coupled to plausible SEDs (Blain et al., 1999b; Trentham et al., 1999; Dunne et al., 2000), clearly indicates that the luminosity function of distant dusty submm galaxies is much greater than that of low-redshift IRAS galaxies (Saunders et al., 1990; Soifer and Neugebauer, 1991), and undergoes very strong evolution. An extrapolation of the low-redshift luminosity function without evolution predicts a surface density of galaxies brighter than $5 \mathrm{mJy}$ 


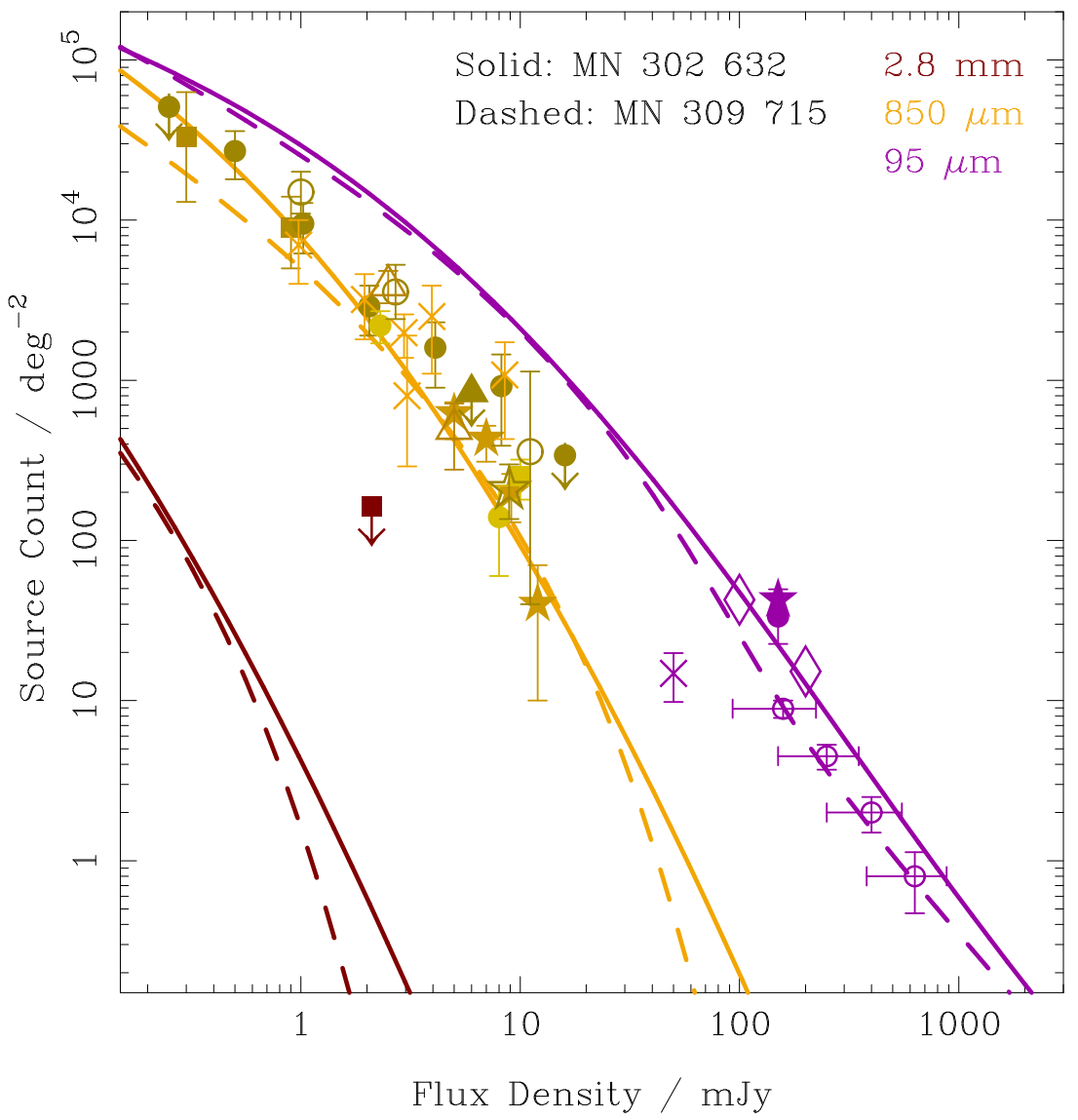

Fig. 9. A summary of count data from several $\mathrm{mm}$, submm and far-IR surveys. The overplotted curves are derived in models that provide good fits to the compilation of data, and are updated from the results in the listed MNRAS papers (Blain et al., 1999b, c). Identical symbols represent post-1999 data from the same source. The errors are shown as $1 \sigma$ values unless stated. The $2.8 \mathrm{~mm}$ data (square) is from Wilner and Wright (1997). At $850 \mu \mathrm{m}$, in order of increasing flux (less than $15 \mathrm{mJy}$ ), data is from Blain et al. (1999a), Cowie et al. (2002) with 90\% confidence limits; Hughes et al. (1998), Chapman et al. (2001b), Barger et al. (1999a, 1998), Smail et al. (1997), Eales et al. $(1999,2000)$ consistent with the increased area reported by Webb et al. (2002a), Borys et al. (2001), Barger et al. (2000) and Scott et al. (2002). The data points between about 2 and $10 \mathrm{mJy}$ are consistent with a steep integral source count $N(>S) \propto S^{\alpha}$, with a power-law index $\alpha \simeq-1.6$. The counts at brighter flux densities are likely to steepen considerably; note that the counts must turn over at fainter flux densities to have $\alpha<-1$ to avoid the background radiation intensity diverging. At $95 \mu \mathrm{m}$, the data is from Juvela et al. (2001), Kawara et al. (1998), Matsuhara et al. (2000), Serjeant et al. (2001) and Linden-Vornle et al. (2000).

at $850 \mu \mathrm{m}$ of only about $0.25 \mathrm{deg}^{-2}$, as compared with the observed density of several $100 \mathrm{deg}^{-2}$ (Fig. 9). Because of the flat flux density-redshift relation in the submm shown in Fig. 4, a 5-mJy SCUBA galaxy at any moderate or high redshift $(z>0.5)$ has a luminosity greater than about $8 \times 10^{12} \mathrm{~L}_{\odot}$. Immediately, this tells us that the comoving density of high-redshift galaxies with luminosities in excess of about $10^{13} \mathrm{~L}_{\odot}$ is 400 times greater than at $z=0$. 


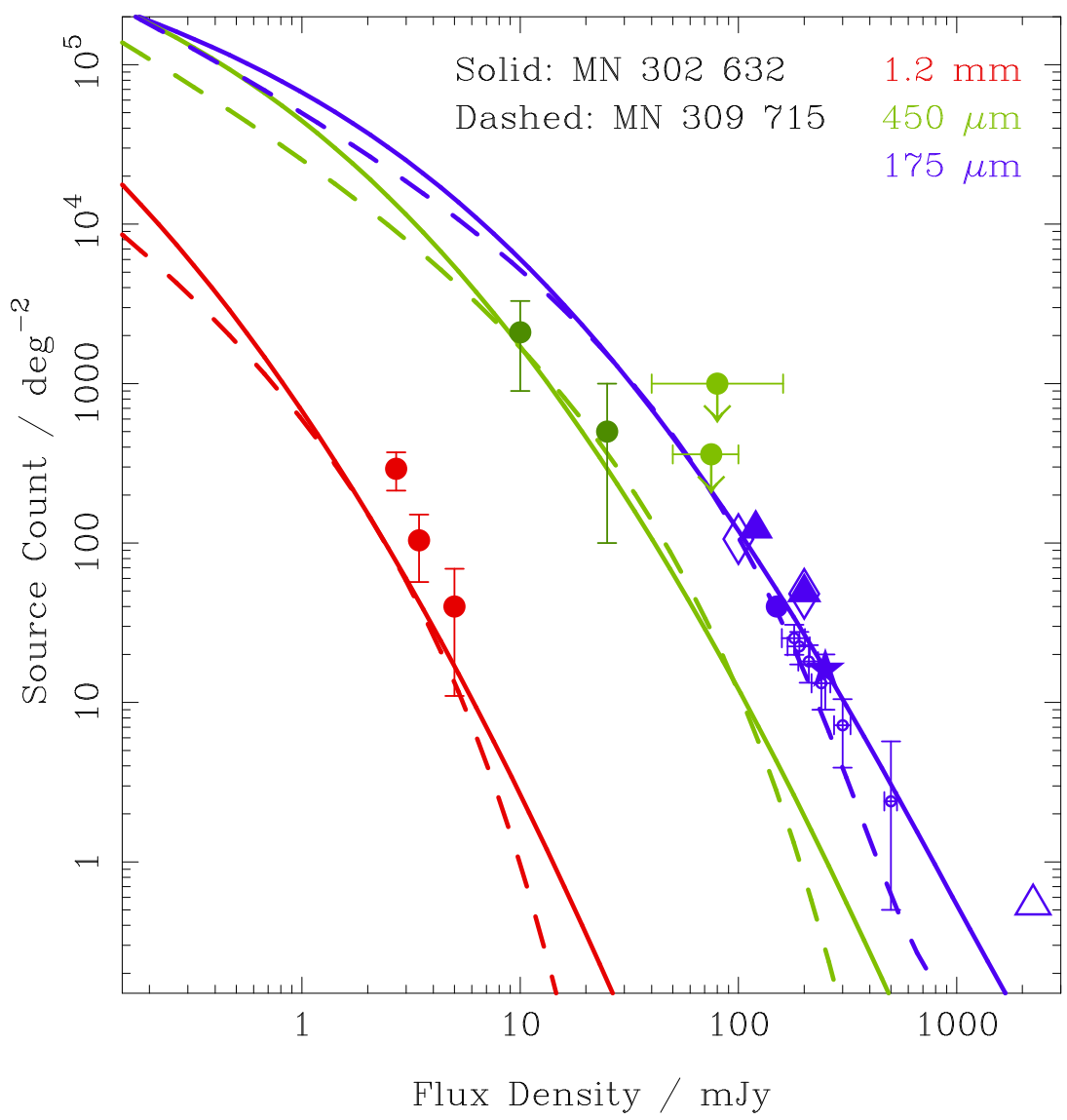

Fig. 10. Counterpart to Fig. 9 for three other observing bands. The data at $1.2 \mathrm{~mm}$ (circles at flux densities less than $10 \mathrm{mJy}$ ) are from Bertoldi et al. (2001), Carilli et al. (2000) and Carilli (2001). The data at $450 \mu \mathrm{m}$ (circles at $10-50 \mathrm{mJy}$ ) are from Smail et al. (2002), with limits from Smail et al. (1997) and Barger et al. (1998). The data at $175 \mu \mathrm{m}$ ( $100 \mathrm{mJy}$ ) are from Kawara et al. (1998), Puget et al. (1999), Matsuhara et al. (2001), Juvela et al. (2001), Dole et al. (2001) and Stickel et al. (1998).

We stress that the submm $K$ correction ensures that the redshift has little effect on the results: the count would be approximately the same whether the population is concentrated at $z \simeq 1$ or extends from $z \simeq 2$ to 10 .

This estimate is subject only to an uncertainty in the dust temperature, which is assumed to be about $40 \mathrm{~K}$. Even if the dust temperature of some of the galaxies is as low as the $20 \mathrm{~K}$ found for low-redshift spiral galaxies, then their luminosity is still about $8 \times 10^{11} \mathrm{~L}_{\odot}$, considerably greater than the several $10^{10} \mathrm{~L}_{\odot}$ expected for typical spiral galaxies. This issue can be addressed by taking into account both the observed background spectrum and the counts at different wavelengths.

The submm-wave background radiation spectrum can also be exploited to provide information about the form of evolution of the luminosity function. The submm-wave background, measured directly using COBE-FIRAS (Puget 


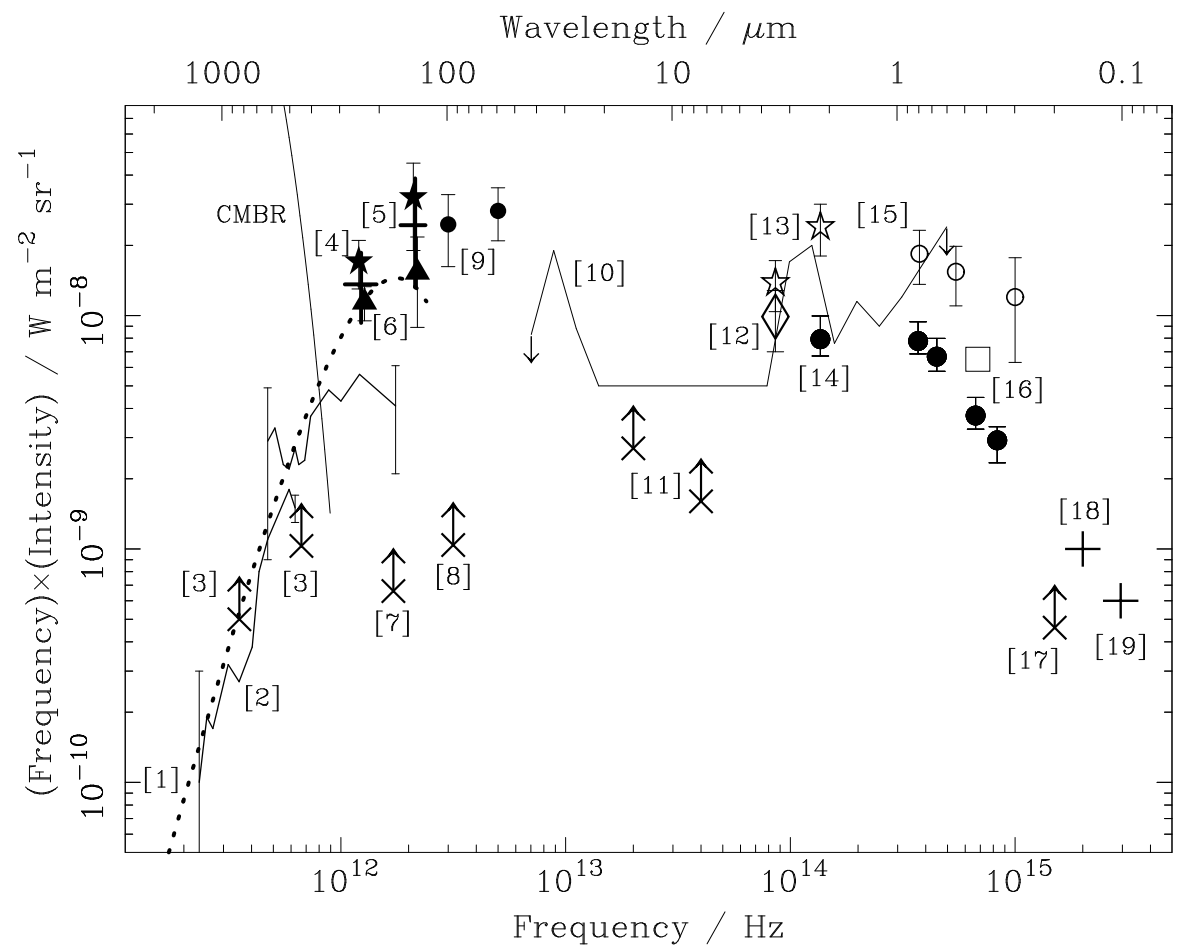

Fig. 11. The observed intensity of cosmic background radiation between the radio and far-UV wavebands. The great majority of the background energy density in the Universe derived from sources other than the CMB is represented in this figure. Almost all of the rest appears in the X-ray waveband. Some significant uncertainty remains, but the combination of measurements and limits indicates that a comparable amount of energy is incorporated in the far-IR background, which peaks at a wavelength of about $200 \mu \mathrm{m}$, and in the near-IR/optical background, which peaks at a wavelength between 1 and $2 \mu \mathrm{m}$. The data originates from a wide range of sources: 1. Fixsen et al. (1998); 2. Puget et al. (1996); 3. Blain et al. (1999a); 4. Schlegel et al. (1998); 5. Hauser et al. (1998); 6. Lagache et al. (2000a) see also Kiss et al. (2001); 7. Puget et al. (1999); 8. Kawara et al. (1998); 9. Finkbeiner et al. (2001); 10. Stanev and Franceschini (1998); 11. Altieri et al. (1999); 12. Dwek and Arendt (1998); 13. Wright and Johnson (2002); 14. Pozzetti et al. (1998); 15. Bernstein (1999) and Bernstein et al. (2002); 16. Toller et al. (1987); 17. Armand et al. (1994); 18. Lampton et al. (1990); and 19. Murthy et al. (1999). For a detailed review of cosmic IR backgrounds see Hauser and Dwek (2001). Note that Lagache et al. (2000a,b) claim that the Finkbeiner et al. points (9) could be affected by diffuse zodiacal emission. Where multiple results are available in the literature the most sensitive result is quoted.

et al., 1996; Hauser et al., 1999; Schlegel et al., 1999), reasonably exceeds the sum of the measured flux densities of discrete galaxies detected in SCUBA surveys (Smail et al., 1997, 2002; Blain et al., 1999a). However, the submm background makes up only a small fraction of the total energy density in the far-IR background, which peaks at a wavelength of about $200 \mu \mathrm{m}$ and is generated by galaxies at redshift $z \sim 1$. The relatively flat source SEDs and the rate of change of the cosmic volume element at this redshift conspire to 
generate most of the background light, just as in the radio, X-ray, optical and near-IR wavebands. The $\mathrm{mm}$ and submm background radiation is unique in originating at a higher redshift. Very little of the background is expected to be generated at $z<1$, and so it is an important signature of high-redshift galaxy formation. Despite representing only a small fraction of the total energy density in the cosmic background radiation, the mm-wave background is one of the cleanest measures of activity in the distant Universe.

There are significant consequences for the evolution of galaxies at high redshifts due to the observed smooth power-law form of the background spectrum, $I_{\nu} \propto \nu^{2.64}$, for $\nu<500 \mathrm{GHz}$ (Fixsen et al., 1998), which originates at moderate to high redshifts, on account of the submm-wave $\mathrm{K}$ correction. The shape of the background radiation spectrum at frequencies greater than about $100 \mathrm{GHz}$ can be approximated quite accurately by associating an evolving comoving volume emissivity ( $\rho_{\mathrm{L}}$ in units of $\mathrm{W} \mathrm{m}^{-3}$ ) with an SED that peaks at a single frequency $\nu_{0}$, so that $\epsilon_{\nu} \propto \rho_{\mathrm{L}}(z) \delta\left(\nu-\nu_{0}\right)$ (Blain and Longair, 1993b), and then integrating over cosmic volume over a fixed angle on the sky. If the SED, via $\nu_{0}$, is assumed not to evolve strongly with redshift - there is no clear evidence that it does - then in order to reproduce the observed slope of the $\mathrm{mm} / \mathrm{submm}$ background spectrum, $\rho_{\mathrm{L}}(z) \propto(1+z)^{\simeq-1.1}$ is required for $z \gg 1$, and so the comoving luminosity density of dust-enshrouded galaxies must decline at large redshifts. If it did not decline, then the background spectrum measured by $C O B E$ would be too flat, with too much energy appearing at long wavelengths. This argument has been made using Monte-Carlo simulations of $\rho_{\mathrm{L}}(z)$ by Gispert et al. (2000). A similar set of simulations have been carried out by Eales et al. (2000), taking into account the observed background radiation spectrum, counts and inferred redshift distribution of submm-selected galaxies.

An approximately equal fraction of the cosmic background radiation energy density emerges in the near-IR/optical and far-IR wavebands (Fig. 11). Because dusty galaxies do not dominate the total volume emissivity at low redshifts (Sanders, 1999; Yun et al., 2001), then the volume emissivity of dusty galaxies must increase by a factor of at least 10, matching the significant evolution of the population of galaxies observed in the optical waveband at $z<1$ (Lilly et al., 1996), to avoid the intensity of the far-IR background radiation being significantly less than observed. Only a very small fraction of the total far-IR luminosity from all low-redshift galaxies comes from galaxies more luminous than $10^{12} \mathrm{~L}_{\odot}$, yet as discussed above in the context of the submm-wave counts, these luminous galaxies are much more numerous at high redshifts, by a factor of several hundred. These twin constraints demand that the form of evolution of the luminosity function of dusty galaxies cannot be pure density evolution, a simple increase in the comoving space density of all far-IR-luminous galaxies. If the counts were to be reproduced correctly in such a model, then the associated background radiation spectrum would be much 
greater than observed. A form of evolution similar to pure luminosity evolution, in which the comoving space density of galaxies remains constant, but the value of $L^{*}$, the luminosity that corresponds to the knee in the luminosity function, increases - in this case by a factor of order 20 - is consistent with both the submm-wave counts and background intensity.

By a more rigorous process, taking into account all available information, including the need to normalize the results to the observed low-redshift population of dust-enshrouded galaxies from the IRAS luminosity function and the populations of galaxies observed by $I S O$ at $z \simeq 1$, the evolution of the luminosity density $\rho_{\mathrm{L}}$ can be constrained. The results have been discussed by Blain et al. (1999b, c), as updated in Smail et al. (2002), and by Eales et al. (2000). They are discussed further in Section 5 below.

\subsection{Confusion}

Source confusion, the contribution to noise in an image due to the superimposed signals from faint unresolved sources clustering on the scale of the observing beam (Condon, 1974; Scheuer, 1974), is a significant problem for observations in the submm waveband (Blain et al., 1998; Eales et al., 2000; Hogg, 2001). This is due to the relatively coarse ( $\simeq 10$ arcsec) spatial resolution currently available. In fact, a significant fraction of the noise in the deepest 850- $\mu \mathrm{m}$ SCUBA image of the HDF-N (Hughes et al., 1998) can be attributed to confusion (Peacock et al., 2000). At present, the practical confusion limit for galaxy detection in SCUBA observations at the atmospherically favored $850-\mu \mathrm{m}$ wavelength is about $2 \mathrm{mJy}$. This limit makes it difficult to determine accurate sub-arcsec positions for the centroids of the submm emission from faint SCUBA-selected galaxies, rendering follow-up observations more challenging. Unfortunately, experience has shown that many known high-redshift galaxies, especially optically-selected LBGs, are typically fainter than the confusion limit, and so are difficult to study using SCUBA.

The variety of count data for dusty galaxies shown in Figs. 9 and 10 can be used to estimate the effect of source confusion in observations made at a wide range of frequencies and angular scales.

The distribution of flux density values from pixel to pixel in an image due to confusion noise depends on the underlying counts of detected galaxies. Confusion noise is always an important factor when the surface density of sources exceeds about 0.03 beam $^{-1}$ (Condon, 1974). The results of a confusion simulation for the 14-arcsec SCUBA $850-\mu \mathrm{m}$ beam are shown in Fig. 12: see also Hogg (2001), Eales et al. (2000) and Scott et al. (2002). Note that Eales et al. assume a very steep count and obtain larger values of confusion noise than 

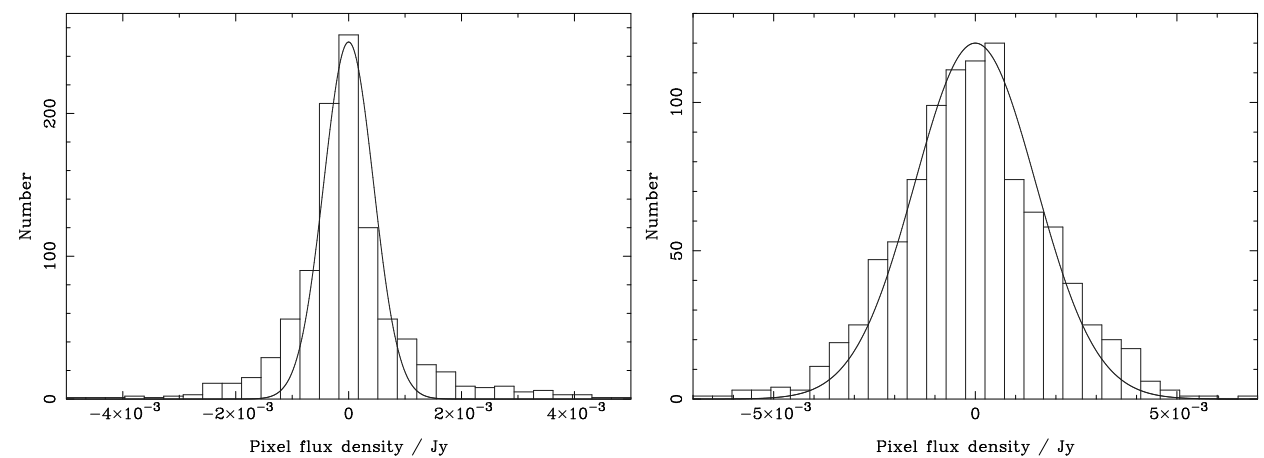

Fig. 12. Histograms showing the simulated effects of confusion noise in deep SCUBA integrations at $850 \mu \mathrm{m}$. Left: the expected distribution of pixel flux densities when the telescope samples the sky in a standard $(-0.5,1,-0.5)$ chopping scheme, with no additional noise terms present. The flux distribution is non-Gaussian, with enhanced high- and low-flux tails as compared with the overplotted Gaussian, which has the width predicted by simple calculations (Fig. 13). Right: the same confusion noise distribution is shown in the right-hand panel, but convolved with Gaussian instrument and sky noise with an RMS value of $1.7 \mathrm{mJy}$, which is typical of the noise level in the SCUBA Lens Survey (Smail et al., 2002). At this noise level, the additional effect of confusion noise is small.

those shown here. Observations made with finer beams at the same frequency suffer reduced confusion noise, while for those made in coarser beams the effects are more severe: compare the results for the much larger 5 -arcmin beam in the three highest frequency submm-wave observing bands of the planned Planck Surveyor space mission all-sky survey shown in Blain (2001a).

The simulated confusion noise distribution is non-Gaussian (Fig. 12), but can be represented quite accurately by a log-normal distribution, leading to many more high-flux-density peaks in an image than expected assuming a Gaussian distribution of the same width. The width of the central peak of the distribution in flux density is approximately the same as the flux density at which the count of sources exceeds 1 beam $^{-1}$. This provides a useful indication of the angular scales and frequencies for which confusion noise is likely to be significant, and of the limit imposed to the effective depth of surveys by confusion for specific instruments: see Fig. 13.

\subsubsection{Confusion and follow-up observations of submm galaxies}

The real problem of confusion for identifying and conducting multiwaveband studies of submm-selected galaxies is illustrated by the results of the first generation of surveys. The very deepest optical image that matches a submm-wave survey is the HDF-N, in which there are several tens of faint optical galaxies (at $R>26$ ) that could be the counterpart to each SCUBA detection (Hughes et al., 1998; Downes et al., 1999a). It is thus impossible to be certain that a correct identification has been made from the submm detection image and 

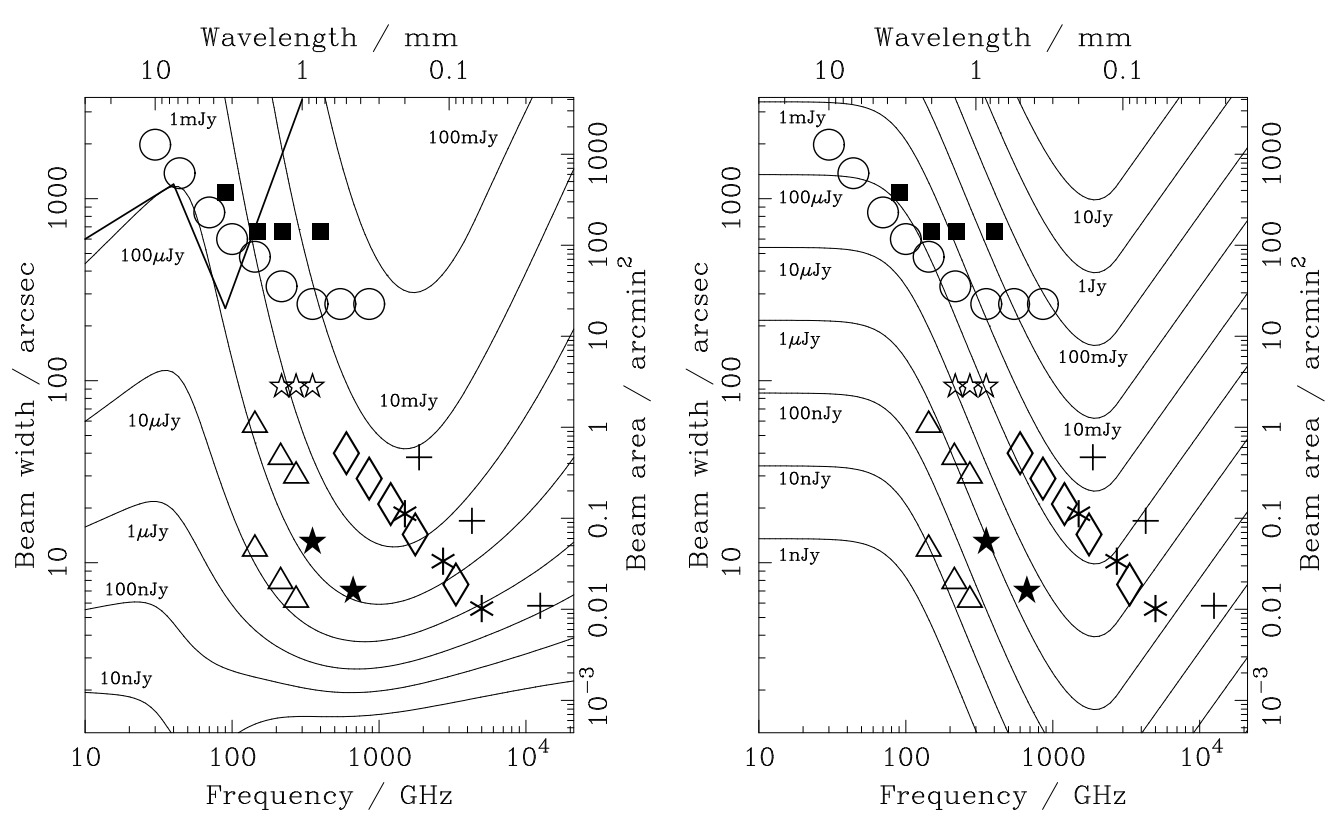

Fig. 13. An approximate measure of the 1- $\sigma$ confusion noise expected as a function of both observing frequency and angular scale from the $\mathrm{mm}$ to mid-IR waveband (updated from Blain et al., 1998). The contributions from extragalactic and Galactic sources are shown in the left and right panels respectively. Radio-loud AGN may make a significant contribution to the top left of the jagged solid line (Toffolatti et al., 1998). A Galactic cirrus surface brightness of $B_{0}=1 \mathrm{MJysr}^{-1}$ at $100 \mu \mathrm{m}$ is assumed. The ISM confusion noise is expected to scale as $B_{0}^{1.5}$ (Helou and Beichman, 1990; Kiss et al., 2001). The bands and beamsizes of existing and future experiments (see Tables 1 and 2) are shown by: circles — Planck Surveyor; squares - BOOMERANG; empty stars - the SuZIE mm-wave Sunyaev-Zeldovich instrument; triangles - BOLOCAM, as fitted to CSO (upper 3 points) and the 50-m Large Millimeter Telescope (LMT; lower 3 points); filled stars — SCUBA (and SCUBA-II); diamonds - Herschel; asterixes - Stratospheric Observatory for Infrared Astronomy (SOFIA); crosses - SIRTF. The resolution limits of the interferometric experiments ALMA and SPECS lie far below the bottom of the panels. The confusion performance of the 2.5-m aperture BLAST balloon-borne instrument is similar to that of SOFIA. Confusion from extragalactic sources is expected to dominate over that from the Milky Way ISM for almost all of these instruments.

optical data alone: compare the identifications in Smail et al. (1998a, 2002). In some cases, EROs and faint non-AGN radio galaxies (Smail et al., 1999, 2000; Gear et al., 2000; Lutz et al., 2001) can be associated with submm galaxies, especially after higher-resolution mm-wave interferometry observations have provided more accurate astrometry for the submm detection (Downes et al., 1999; Frayer et al., 2000; Gear et al., 2000; Lutz et al., 2001), to reduce the effects of submm confusion, with the investment of significant amounts of observing time. The surface density of both EROs and faint non-AGN radio galaxies is less than that of the faintest optical galaxies, and so the probability of a chance coincidence between one and a submm galaxy is reduced. A very red color and detectable radio emission from a high-redshift galaxy are 
both likely to indicate significant star-formation/AGN activity and/or dust extinction, making such galaxies better candidate counterparts even in the presence of confusion-induced positional uncertainties.

\subsection{Multi-waveband follow-up studies}

A great deal of telescope time has been spent so far to detect and study submm-selected galaxies in other wavebands. In many cases, rich archival data predated the submm observations: most notably in HDF-N (Hughes et al., 1998). Considerable data was also available in the fields of rich clusters (Smail et al., 1997, 1998a, 2002; Cowie et al., 2002), in the region of the Eales et al. (1999, 2000) surveys in Canada-France Redshift Survey (CFRS) fields, which include the Groth Strip, and in the deep Hawaii survey fields (Barger et al., 1999a). The results of follow-up deep optical and near-IR imaging (Frayer et al., 2000) and spectroscopy (Barger et al., 1999b), mm-wave continuum imaging (Downes et al., 1999a; Bertoldi et al., (2000); Frayer et al. 2000; Gear et al. 2000; Lutz et al., 2001; Dannerbauer et al., 2002) and molecular line spectroscopy (Frayer et al., 1998, 1999; Kneib et al., 2002) have been published, and many additional studies are under way. The time spent following up the SCUBA Lens Survey (Smail et al., 2002) exceeds by almost an order of magnitude the time required to make the submm discovery observations (Smail et al., 1997). The difficulty of the task is highlighted by the identification of plausible counterparts to these galaxies being only about $60 \%$ complete over $4 \mathrm{y}$ later at the start of 2002. The follow-up results from a well-studied subset of galaxies in the SCUBA Lens Survey are shown in Figs. 14-20, in order of decreasing $850-\mu \mathrm{m}$ flux density. These are chosen neither to be a representative sample of submm galaxies, nor to be a sufficiently large sample for statistical studies, but rather to present a flavor of the range of galaxies that can be detected in submm-wave surveys for which high-quality multi-waveband data is available. The galaxies that are presented are chosen to have good positional information, and redshifts where possible. Other detections for which excellent multi-wavelength follow-up data are available include the brightest source in the HDF-N SCUBA image (Hughes et al., 1998; Downes et al., 1999a), an ERO detected in the CUDSS survey by Eales et al. (1999) (Gear et al., 2000), a $z=2.8$ QSO in a cluster field (Kraiberg-Knudsen et al., 2001), and the substantially overlapping catalogs of galaxies detected by Cowie et al. (2002) in deeper images of 3 of the 7 clusters in the Smail et al. lens survey.

\subsubsection{Optical/near-IR}

The properties of submm galaxies in the optical waveband, corresponding to the rest-frame UV waveband, appear to be very diverse (Ivison et al., 2000a). 
This may be due in part to their expected broad redshift distribution. However, given that two submm galaxies at the reasonably high redshifts $z=2.5$ and 2.8 are known to be readily detectable at $B \simeq 23$ (Ivison et al., 1998, 2000, 2001) - before correcting each for the magnitude (factor of about 2.5) of amplification due to the foreground cluster lens - while most others are very much fainter (Smail et al., 2002; Dannerbauer et al., 2002), it is likely that much of the spread in their observed properties is intrinsic. As most counterparts are extremely faint, confirmation of their nature requires a large, completely identified sample of submm galaxies with known redshifts, which is likely to be some time away. It is possible that optically faint submm galaxies have similar properties, an issue that can be addressed when deep near-IR observations are available.

Ivison et al. (2000a) and Smail et al. (2002) proposed a 3-tier classification system to stress the varied nature of submm galaxies (three being an eminently sensible number of classes for 15 galaxies!). Class-0 galaxies are extremely faint in both the observed optical and near-IR wavebands. Class-1 galaxies are EROs, very faint in the optical but detectable in the near-IR, while Class2 galaxies are relatively bright in both bands. It is unclear how closely this scheme reflects the underlying astrophysics of the submm galaxies; however, the classification separates the optically bright galaxies (Class 2s), for which the acquisition of optical redshifts and confirming $\mathrm{CO}$ redshifts are likely to be practical, and the fainter galaxies, for which this will be a great challenge (Class 0s). A similar approach for MAMBO sources has been discussed by Dannerbauer et al. (2002). Note that submm galaxies could change classification by having different redshifts, despite identical intrinsic SEDs.

\subsubsection{Ultradeep radio images}

The surface density of the faintest radio sources that can be detected using the VLA (Richards, 2000) is significantly less than that of optical galaxies, and so an incorrect radio counterpart to a submm-selected galaxy is relatively unlikely to be assigned by chance. If 1.4-GHz VLA images are available at a flux limit approximately 1000 times deeper than $850-\mu \mathrm{m}$ images, then the radio counterparts to non-AGN submm galaxies should be detectable to any redshift (see Fig. 7). Deep radio follow-up observations of submm-selected galaxies yielding cross identifications have been discussed by Smail et al. (2000), and further information about very faint radio sources in the field of the UK 8-mJy SCUBA survey (Fox et al., 2002; Scott et al., 2002) should soon be available in Ivison et al. (2002). Despite an extremely deep radio image (Richards, 2000), the brightest submm galaxy detected in HDF-N (Hughes et al., 1998) does not have a radio detection, probably indicating a very high redshift. The survey results reported by Eales et al. (2000) and Webb et al. (2002a) were discussed in the context of radio data covering the same fields; however, the radio survey 
is not deep enough to detect a significant fraction of the relatively faint submm sources. As can be seen for the specific submm galaxies shown in Figs. 14-20, when they are available, deep high-resolution radio images are very useful for determining accurate positions and even astrophysical properties of submm galaxies: see Ivison et al. (2001).

\subsubsection{CO rotation line emission and continuum mm-wave interferometry}

The detection of CO line emission from submm-selected galaxies is a crucial step in the confirmation of their identification. It has been demonstrated in only three cases so far (see Figs. 14, 18 and 19), using the OVRO Millimeter Array (MMA; Frayer et al., 1998, 1999), in one case in combination with the BIMA array (Ivison et al., 2001), and the IRAM PdBI (Kneib et al., 2002). These observations are very time-consuming, typically requiring tens of hours of observing time. The $\simeq 1 \mathrm{GHz}$ instantaneous bandwidth of existing linedetection systems also means that a redshift accurate to at least $0.5 \%$ must be known before attempting a CO detection. In other cases, continuum emission is detected using the interferometers, confirming the reality of the initial submm detection and providing a better position (Downes et al., 1999a; Bertoldi et al., 2000; Frayer et al., 2000; Gear et al., 2000; Lutz et al., 2001; Dannerbauer et al., 2002), but no absolute confirmation of a correct optical/near-IR identification or a crucial redshift.

The ALMA interferometer array will have the collecting area and bandwidth to make rapid searches for CO line emission in the direction of known submm continuum sources from about 2010. Specialized wide-band mm-wave spectrographs to search for multiple high-redshift CO lines separated by $115 \mathrm{GHz} /(1+$ $z$ ) that are currently under development (Glenn, 2001). Wide-band cm-wave receivers at the 100-m clear-aperture Green Bank Telescope (GBT) could detect highly redshifted $115-\mathrm{GHz} \mathrm{CO}(1 \rightarrow 0)$ line emission.

\subsubsection{X-ray observations}

Based on synthesis models of the X-ray background radiation intensity (Fabian and Barcons, 1992, Hasinger et al., 1996), Almaini et al. (1999) and Gunn and Shanks (2002) suggested that 10-20\% of the submm galaxy population could be associated with the hard X-ray sources that contribute this background.

Observations of fields with common deep Chandra and SCUBA images were discussed in Section 2.8.

The small degree of observed overlap between the submm and X-ray sources, implies that if a significant fraction of submm galaxies are powered by accretion in AGN, then the accretion must occur behind an extremely thick absorbing column of gas, and less than $1 \%$ of the X-ray emission from the 
AGN can be scattered into the line of sight (Fabian et al., 2000; Barger et al., 2001; Almaini et al., 2002). In order to avoid detection using SCUBA, high-redshift hard X-ray Chandra sources must either contain a very small amount of gas and dust, and thus have only a small fraction of their energy reprocessed into the far-IR waveband, which seems unlikely given their hard spectra; or they must contain dust at temperatures much higher than appears to be typical for submm-selected galaxies. The detection of Chandra sources in Abell 2390 using $I S O$ at $15 \mu \mathrm{m}$, but not using SCUBA at $850 \mu \mathrm{m}$, argues in favor of at least some Chandra sources having very hot dust temperatures (Wilman et al., 2000). Comparison of larger, deep ISO 15- $\mu$ m images with Chandra images shows that most of the faint, red AGN detected by Chandra are detected in the mid-IR (Franceschini et al., 2002).

This should be readily confirmed using wide-field sensitive mid-IR observations of Chandra fields using SIRTF, images which will also yield well-determined SEDs for the dust emission from the detected galaxies.

\subsubsection{Mid- and far-IR observations}

Distant submm galaxies are too faint at far- and mid-IR wavelengths to have been detected in the all-sky IRAS survey. However, the first submm-selected galaxies were detected while the next-generation $I S O$ space observatory was still operating, and there were both late-time ISO observations of submm fields, and some serendipitous overlap of fields. In general, the small aperture and small-format detector arrays of $I S O$ still led to relatively little overlap between SCUBA and ISO galaxies, for example in the HDF-N (Hughes et al., 1998; Elbaz et al., 1998) and Abell 2390 (Fabian et al., 2000; Wilman et al., 2000). The brightest SCUBA galaxies in the cluster Abell 370 (Ivison et al., 1998a) have fluxes measured by $I S O$ at $15 \mu \mathrm{m}$ (Metcalfe, 2001), providing valuable, and otherwise difficult to obtain, constraints on their short-wavelength SEDs (Fig. 2), and thus their dust temperatures. The much larger detector arrays aboard $S I R T F$ should allow many more constraints to be imposed on the mid-IR SEDs of submm galaxies. Existing deep submm fields are included for imaging within the SIRTF guaranteed time programs, and individual submm galaxies have been targeted for mid-IR spectroscopy.

\subsection{A gallery of follow-up results}

In this section we show some of the submm-selected galaxies with the most complete and comprehensive follow-up information, including all three with confirmed redshifts (Figs. 14, 18 and 19). To reveal more of the diversity of

\footnotetext{
${ }^{13}$ Details of SIRTF observing programs can be found at sirtf.caltech.edu.
} 
counterparts to the SCUBA galaxies, we also show a relatively strong radio source with a faint red $K$-band counterpart (Fig. 17), two $K \simeq 19.5$ galaxies, one a formal ERO and the other with very red colors (Smail et al., 1999; Figs. 16 and 20), which are likely to be the correct counterpart on the grounds of the relatively low surface density of EROs; and a mm-continuum source located by the OVRO MMA at the position of a SCUBA-selected galaxy, with a very faint K-band counterpart observed using the NIRC instrument on the Keck telescope (Frayer et al., 2000; Fig. 15). Similar enigmatic faint red galaxies have been reported as counterparts to well-located submm-selected galaxies from other submm surveys by Gear et al. (2000) and Lutz et al. (2001), while Dannerbauer et al. (2002) do not find counterparts to three well-located MAMBO galaxies to a $3-\sigma$ limit of $K_{\mathrm{s}}=21.9$.

The galaxies shown in Figs. 14-20 are certainly an unrepresentative sample of submm-selected galaxies, missing galaxies that are either intrinsically very faint at other wavelengths or lie at the highest redshifts. It is to be hoped that within the next few years, deep follow-up observations, especially nearIR ground-based observations ${ }^{14}$ and mid-IR observations using SIRTF will reveal the nature of the majority of submm-selected galaxies. 10

\subsection{Clustering properties}

The deep submm-wave surveys made to date provide relatively little information about the spatial distribution of the detected galaxies. There is some indication from the UK 8-mJy SCUBA survey (Almaini et al., 2002; Fox et al., 2002; Ivison et al., 2002; Scott et al., 2002) and from the widest-field MAMBO surveys (Carilli et al., 2001) that the clustering strength of SCUBA-selected galaxies is greater than that of faint optically-selected galaxies, yet less than that of $K \simeq 20$ ERO samples (Daddi et al., 2000). Webb et al. (2002b) point out that the angular clustering signal expected in submm surveys is likely to be suppressed by smearing in redshift, as the submm galaxies should have a wide range of redshifts, and so the spatial correlation function might in fact be stronger than that of the EROs. As most $K \simeq 20$ EROs are evolved elliptical galaxies at $z \simeq 1$, which are likely to be amongst the first galaxies to form in the most overdense regions of the Universe, their strong clustering is easily explained. However, a definitive result on the clustering of submm galaxies awaits a much larger sample of galaxies than considered by Scott et al. (2002) and Webb et al. (2002b). Characteristic brightness fluctuations on the angular scales expected from faint unresolved dusty galaxies have been found in both

$\overline{14}$ Near-IR imaging to $2 \sigma K \simeq 24$ of all of the SCUBA Lens Survey submm galaxies is underway using the NIRC camera at the Keck telescopes: see Fig. 15.

${ }^{15}$ More information about this sample can be found in the catalog paper of the SCUBA Lens Survey (Smail et al., 2002 and references therein). 

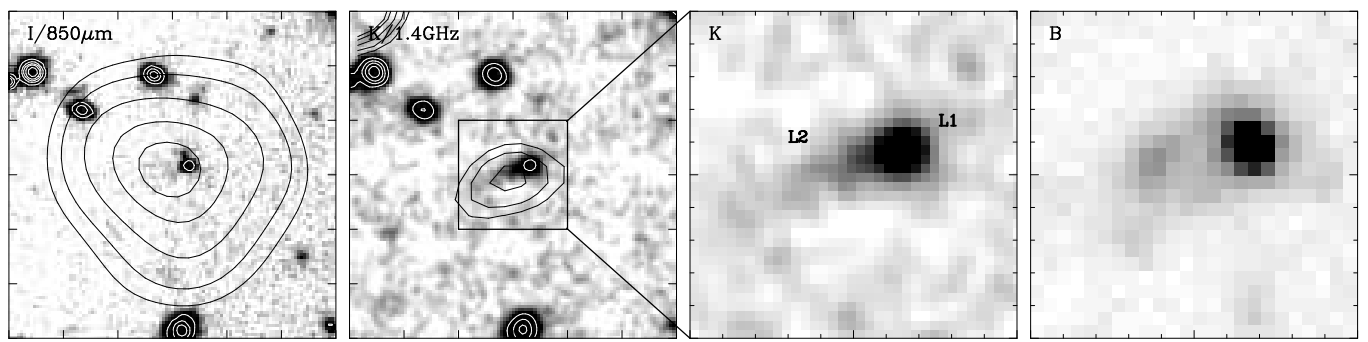

Fig. 14. Multi-waveband images of SMM J02399-0136 (23 mJy; Ivison et al., 1998; Frayer et al., 1998). The format of this figure is the template for those that follow. Note that these multiwaveband figures are presented in order of reducing $850-\mu \mathrm{m}$ flux density, without correcting for gravitational lensing amplification. The leftmost panel shows black contours of $850-\mu \mathrm{m}$ emission superimposed on a grayscale $I$-band image. The second panel shows black contours of faint $1.4-\mathrm{GHz}$ radio emission superimposed on a $K$-band image. These two left-hand images are both 30 arcsec on a side. The third panel shows a 10 -arcsec zoom of the $K$-band image (from UKIRT unless otherwise stated; Smail et al., 2002). The rightmost panel shows a $B$-band CFHT image in this figure; in the figures that follow this panel shows an $H S T$ image. Here and in the figures that follow, white contours are added to show contrast in saturated regions of the grayscale. North is up and East is to the left. SMM J02399-0136 is a merging galaxy with a confirmed optical/radio counterpart, and a CO redshift $z=2.808$ : see Vernet and Cimatti (2001) for a new high-quality spectrum showing Lyman- $\alpha$ emission from this galaxy extended over 12 arcsec.
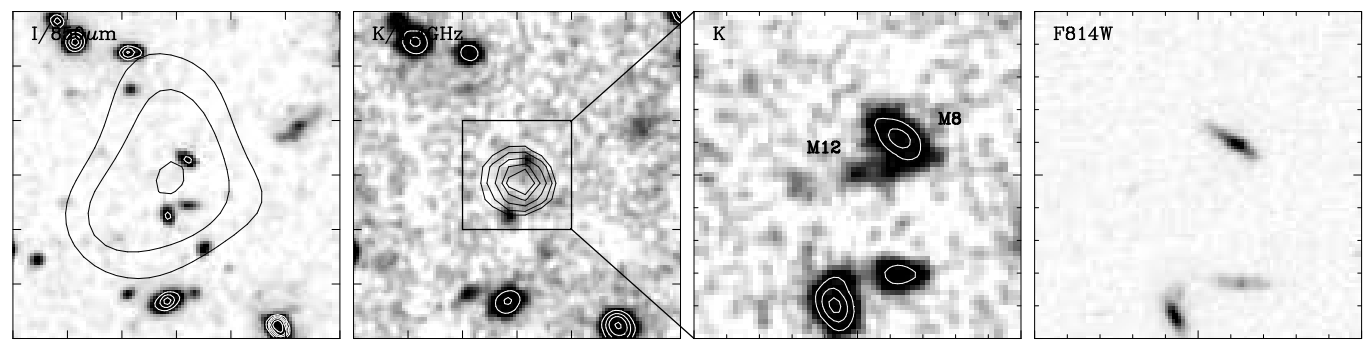

Fig. 15. Images of SMM J00266+1708 (18.6 mJy; Frayer et al., 2000). The left-hand $K$-band image is from UKIRT; the right-hand $K$-band image is from Keck-NIRC. The $K$-band detection is located at the position of the very red galaxy M12 in a 1.1-mm continuum image obtained using the OVRO MMA.
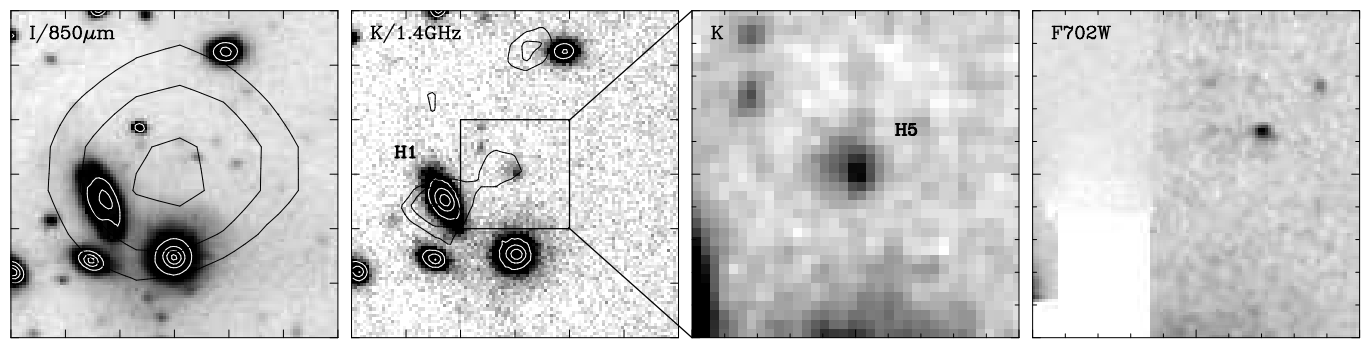

Fig. 16. Images of SMM J0942+4658 (17.2 mJy), an ERO counterpart (Smail et al., 1999). Faint radio emission and extended, rather bright $K$-band emission make this a good candidate for the source of the submm emission. H1 is a low-redshift spiral galaxy in the foreground of Abell 851. 

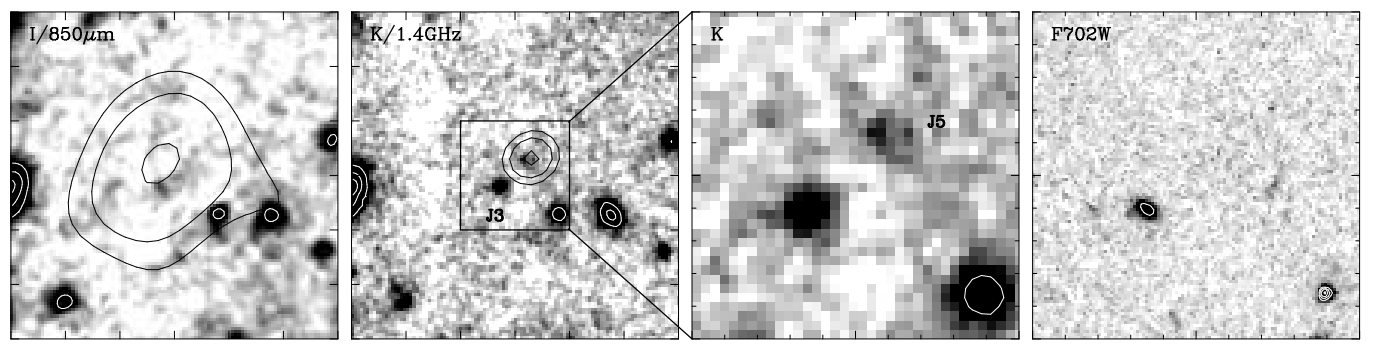

Fig. 17. Multi-waveband images of SMM J14009+0252 (14.5 mJy), the bright radio-detected submm galaxy Abell 1835 (Fig. 1; Ivison et al., 2000). Two faint near-IR counterparts can be seen in the $K$-band image. Of these, J5 is extremely red, has no counterpart in the HST-F702W image, and is aligned accurately with the centroid of the radio emission.
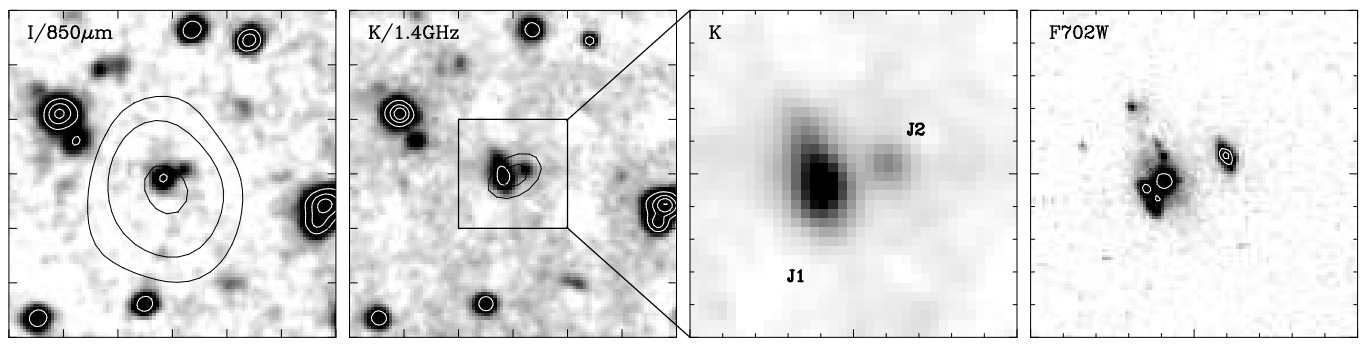

Fig. 18. Multi-waveband images of SMM J14011+0252 (12.3 mJy; Ivison et al., 2000, 2001). This complex merging system has a confirmed optical/radio counterpart, and a CO redshift $z=2.565$ (Frayer et al., 1999). High-resolution CO and radio images are presented in Ivison et al. (2001). Note that the Northern extension of J1 is extremely red, and is close to the centroid of the radio emission. J2 is blue, while $\mathrm{J} 1$ is red. The complexity of this system is a caution against simple treatment of extinction as a uniform screen in submm galaxies: for a detailed discussion see Goldader et al. (2002) and references therein.
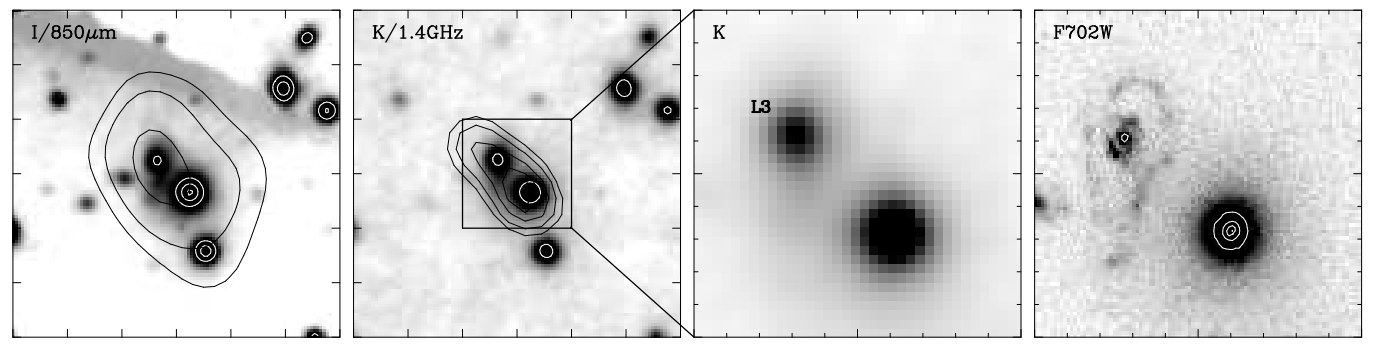

Fig. 19. Multi-waveband images of SMM J02399-0134 (11.0 mJy; Kneib et al., 2002). This ring galaxy has a confirmed optical/radio counterpart, and a CO redshift $z=1.06$. Its low redshift accounts for its very bright $K$-band image and mid-IR $I S O$ detection at $15 \mu \mathrm{m}$. The other galaxy in the $K$-band image is a member of Abell 370 .

the $850-\mu \mathrm{m}$ SCUBA image of the HDF-N (Peacock et al., 2000) and in deep, confused 175- $\mu \mathrm{m}$ ISO images (Lagache and Puget, 2000; Kiss et al., 2001).

Haiman and Knox (2000) have discussed the details of measurements of the correlation function of unresolved submm galaxies on arcminute angular scales 


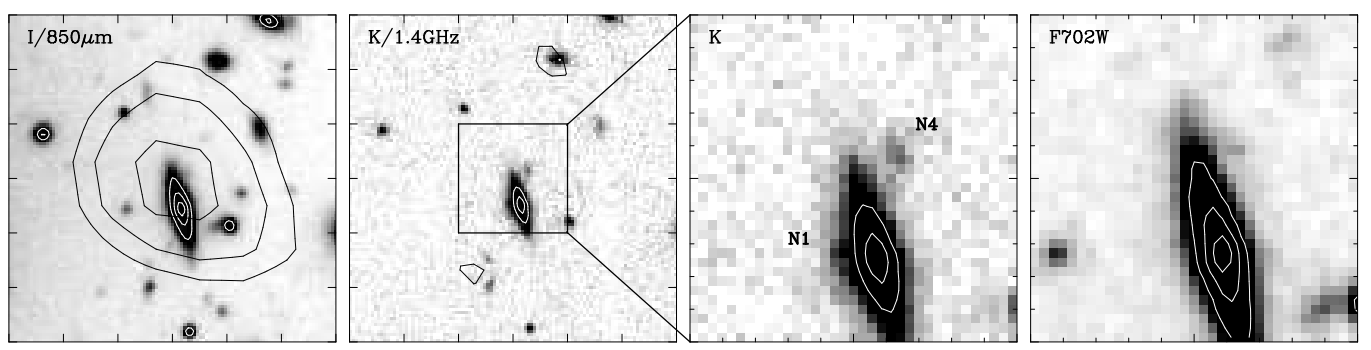

Fig. 20. Images of SMM J04431+0210 (7.2 mJy), a very red counterpart (Smail et al., 1999). A tentative $\mathrm{H} \alpha$ redshift of $z=2.5$ is determined from a near-IR Keck-NIRSPEC observation. Unlike SMM J09429+4658 this galaxy has no radio emission.

in the context of CMB experiments, finding that the correlated signal can carry important information about the nature and evolution of the submm galaxy population. A simpler investigation by Scott and White (1999) drew similar conclusions, while there is further discussion by Magliocchetti et al. (2001).

\section{Submm galaxy luminosity functions and their relationship with other populations}

Submm-selected galaxies are an important component of the Universe, but are typically very faint in other wavebands, and so difficult to study. This immediately implies that the submm population does not overlap significantly with other types of high-redshift galaxies, although it may be possible to infer their properties if detailed information about these other classes is available (Adelberger and Steidel, 2000). The likely lack of overlap is reinforced by the relatively low surface density of submm galaxies as compared with the faintest optically-selected galaxies. Nevertheless, a detailed understanding of the process of galaxy formation demands that the relationship of the submm galaxies to other populations of high-redshift galaxies is determined.

\subsection{Optically-selected Lyman-break galaxies (LBGs)}

The LBGs (Steidel et al., 1999) are sufficiently numerous to have a welldefined luminosity function (Adelberger and Steidel, 2000). The effects of dust extinction on the inferred luminosity of a small subset of LBGs have been estimated reliably from near-IR observations of $\mathrm{H} \alpha$ emission: corrections by factors of 4-7 are indicated (Pettini et al., 1998, 2001; Goldader et al. 2002). At present, it is difficult to confirm this degree of extinction directly, as attempts to detect LBGs using submm-wave instruments have not so far been successful: see Section 2.8. Observations suggest that a typical LBG has a 850- $\mu$ m flux 
density of order $0.1 \mathrm{mJy}$, well below the confusion limit at the resolution of existing submm-wave images.

Adelberger and Steidel (2000) have discussed the various selection effects associated with submm, optical and faint radio selection of high-redshift galaxy samples. They assumed that the relation between the slope of the UV SED of a galaxy and the fraction of its luminosity emitted in the far-IR waveband that is observed for low-redshift IUE starbursts with luminosities less than about $10^{11} \mathrm{~L}_{\odot}$ (Meurer et al., 1999) holds at greater redshifts and luminosities. A common, smooth luminosity function can then account for the properties of LBGs and submm galaxies. A priori, there must be an underlying multiwaveband luminosity function of all high-redshift galaxies from which both classes of galaxies are drawn. However, while observations of some submm galaxies (a key example being SMM J14011+0252; Ivison et al., 2000a, 2001; Fig. 18) seem to support this interpretation at first sight, it is clear that only the J2 region of this galaxy would be identified as a LBG, while the submm emission is concentrated nearer to J1. Further discussion can be found in Goldader et al. (2002). Because of the apparent diversity of optical-submm properties of submm galaxies (Ivison et al., 2000a; Smail et al., 2002), this simple transformation is unlikely to hold. Hence, a fraction of submm galaxies will probably never be detected in rest-frame UV continuum surveys because of their extreme faintness. The confirmed ERO submm galaxies (Smail et al., 1999; Gear et al., 2000; Lutz et al., 2001) are clear examples of such a population.

\subsection{Extremely Red Objects (EROs)}

The development of large format near-IR detectors has enabled relatively deep, wide-field IR surveys, and lead to the discovery of a class of faint EROs (galaxies with colors in the range $R-K>5.5-6$ ), supplementing traditional lowmass stellar EROs (Lockwood, 1970). At first EROs were found one by one, (Hu and Ridgeway, 1994; Graham and Dey, 1996), but statistical samples are now detected in $K<20$ near-IR surveys (Thompson et al., 1999; Yan et al., 2000; Daddi et al., 2000; Totani et al., 2001), in parallel to their identification in multiwaveband surveys (Smail et al., 1999; Pierre et al., 2001; Smith et al., 2001; Gear et al., 2000; Lutz et al., 2001). The clustering of relatively bright $K<19.2$ EROs (Daddi et al., 2000) is observed to be very strong, fueling speculation that EROs are associated with the deepest potential wells that have the greatest density contrast at any epoch. This has been used as an argument in favor of their association with submm galaxies, which could be good candidates for massive elliptical galaxies in formation (Eales et al., 1999; Lilly et al., 1999; Dunlop, 2001). 
There are two obvious categories of extragalactic EROs: very evolved galaxies, containing only cool low-mass stars, and strongly reddened galaxies, with large amounts of dust absorption, but which potentially have a very blue underlying SED. Only the second are good candidates for identification with submm-luminous galaxies. Detections of faint radio emission associated with young supernova remnants in EROs, and determinations of signatures of ongoing star-formation in their rest-frame UV colors should allow these cases to be distinguished. Radio and submm follow-up observations of EROs have tended to show that most are passively evolved non-star-forming galaxies without detectable radio emission (Mohan et al., 2002), with at most about 10-20\% being candidates for dust-enshrouded starbursts/AGN. It is important to remember that few EROs selected from wide-field near-IR surveys, which reach limits of $K \simeq 20$, are actively star-forming submm galaxies (see the summary of results in Smith et al., 2001).

A small but significant fraction of submm galaxies appear to be associated with EROs at bright magnitudes $K<20$ (Smail et al., 1999, 2002). Many more submm galaxies probably fit the ERO color criterion, but at much fainter magnitudes; see for example the $K=22.5$ SCUBA galaxy shown in Fig. 15 (Frayer et al., 2000). It is certainly possible that future fainter ERO samples with $K>22$ could contain a greater fraction of submm-luminous galaxies and fewer passive ellipticals than the $K \simeq 20$ samples. Note also that EROs have unfavorable K corrections for detection at high redshifts (Dey et al., 1999; Gear et al., 2000): beyond $z \simeq 2.5$ any ERO would be extremely faint at even near-IR wavelengths. This could account, in part or in whole, for the extreme faintness of counterparts to submm galaxies, if many do have extremely red intrinsic colors.

\subsection{Faint radio galaxies}

As discussed above, the faintest radio galaxies should be detectable in the submm waveband if the far-IR-radio correlation remains valid at high redshifts. The narrow dispersion of this correlation suggests that submm galaxies and faint radio galaxies are perhaps the most likely populations of high-redshift galaxies to overlap substantially. Surveys made using SCUBA to search for optically-faint, and thus presumably high-redshift, galaxies with radio flux densities close to the detection threshold of the deepest radio surveys (Barger et al., 2000; Chapman et al., 2001b) have been used to detect many tens of high-redshift dusty galaxies much more rapidly than blank-field surveys. The selection effects at work when making a radio-detected, optically-faint cut from a radio survey are not yet sufficiently well quantified to be sure that these catalogs are representative of all submm galaxies. The typical optical magnitudes of the radio-selected objects with submm detections are clustered 
around $I \simeq 24$ and greater. Hence, bright optical counterparts to mJy-level submm galaxies are rare (Chapman et al., 2002b). Because relatively accurate positions are available from the radio observations, it should be possible to determine spectroscopic redshifts for a significant number of these galaxies, providing a valuable contribution to our knowledge of the distances to at least a subset of submm galaxies.

\subsection{Active galaxies and X-ray sources}

An important category of objects that could be associated with submm galaxies are accreting AGN. As discussed in Sections 2.8 and 3.2.4, the overlap between Chandra and SCUBA galaxies does not appear to be very great - at about the $10 \%$ level. However, the gravitational energy released when forming the supermassive black holes in the centers of galactic bulges (Magorrian et al., 1998) is likely to be only a few times less than the energy released by stellar nucleosynthesis over the lifetime of the stars in the bulge, and so a case remains for the existence of both a significant population of Compton-thick AGN submm sources with no detectable X-ray emission at energies less than $10 \mathrm{keV}$, and for hot dusty AGN detectable in the mid- and far-IR but not the submm wavebands (Wilman et al., 2000; Blain and Phillips, 2002).

\subsection{Gamma-ray burst (GRB) host galaxies}

An interesting new development is the idea that if GRBs are likely to be associated with the deaths of massive stars, then the rate of GRBs and the global high-mass star-formation rate should be linked (Krumholz et al., 1998). By searching for submm emission from the directions of GRBs, it may be possible to test whether either submm or UV-bright galaxies are the dominant population to host high-mass stars, and what fraction of the submm galaxies are powered by non-GRB-generating AGN (Blain and Natarajan, 2000). About $10 \%$ of GRBs are expected to be in hosts with $850-\mu \mathrm{m}$ flux densities greater than 5 mJy (Ramirez-Ruiz et al., 2002), if submm galaxies dominate the cosmic star-formation rate and are not typically powered by AGN. Two excellent candidates for submm-loud host galaxies of GRBs are now known (Berger et al., 2001; Frail et al., 2002), the first based on deep VLA radio images, the second on direct SCUBA and MAMBO mm/submm observations. Most GRB hosts appear to be associated with $R \simeq 25$ optical galaxies (Bloom et al., 2002), which could also be typical of the submm galaxy population. It is difficult to detect GRB host galaxies without hitting the confusion limit using SCUBA, but attempts are underway. As a byproduct of surveys for submm afterglow emission, Smith et al. $(1999,2002)$ imposed limits to the submm 
host galaxy emission from the direction of 12 GRBs. An ongoing JCMT program (Nial Tanvir et al.) is searching directly for submm emission from the host galaxies of accurately located GRBs (Barnard et al., 2002). Detecting and resolving the submm emission from GRB host galaxies should ultimately be very simple using ALMA, requiring observations of only a few minutes minutes per burst.

\subsection{Prospects for the follow-up observations in the future}

In order to make detailed submm-wave studies of the astrophysics of highredshift galaxies, high-resolution images will be required. Existing mm-wave interferometers can provide high-quality images of the brightest submm galaxies (for example Frayer et al., 2000; Gear et al., 2000; Lutz et al., 2001); however, in order to detect rapidly typical LBGs, EROs and hard X-ray sources, the additional sensitivity and resolution that should be provided by ALMA is required. Improved information on the SEDs of dusty galaxies will be obtained using the SIRTF, SOFIA, ASTRO-F and Herschel space- and air-borne observatories: see Tables 1 and 2 .

\section{Modeling the evolution of submm galaxies}

As soon as the first submm galaxies were detected in 1997, it was clear that they made a significant contribution to the luminosity density in the highredshift Universe, subject to the plausible assumptions that their SEDs were similar to those of luminous low-redshift dusty galaxies, and that their redshifts were not typically less than about $z=0.5$. These assumptions remain plausible and have been confirmed to an acceptable level by subsequent observations (Smail et al., 2000, 2002). Despite an initial suggestion that 30\% of the SCUBA galaxies could be at $z<1$ (Eales et al., 1999), it now seems that a median redshift of submm-selected galaxies is of order 2-3 (Eales et al., 2000; Smail et al., 2002).

We have already discussed that evolution by a factor of about 20 in the value of $L^{*}$ is required to account for the properties of the submm source counts and background radiation intensity. That is the key result from submm surveys, but how can it be explained in models of galaxy evolution? In this section we will not describe the modeling process in great detail, but we highlight the key features of such analyses, and the most important future tests of our current understanding.

It is important is to be aware that there is still considerable uncertainty in the 
exact form of evolution required to explain the submm observations. While strong luminosity evolution of the dusty galaxy population is required out to $z \simeq 1$ and beyond, the detailed form of that evolution is rather loosely constrained by count and background data. Redshift distributions are an essential requirement in order to determine the form of evolution accurately.

\subsection{An array of possible treatments}

A variety of approaches have been taken to making predictions for and interpreting the results of submm surveys. This work began after the deep $60-\mu \mathrm{m}$ counts of IRAS galaxies were derived, and it was clear that strong evolution was being observed out to $z \sim 0.1$ (Hacking and Houck, 1987; Saunders et al., 1990; Bertin et al., 1997). Observations of more distant galaxies at longer wavelengths could probe the extrapolated form of evolution, and disentangle the degenerate signatures of density and luminosity evolution (Franceschini et al., 1988; Oliver et al. 1992).

Before the first deep submm survey observations in 1997, Franceschini et al. (1991), Blain and Longair (1993a, b, 1996) and Pearson and Rowan-Robinson (1996) made a variety of predictions of what might be detected in submm surveys. Guiderdoni et al. (1998) and Toffolatti et al. (1998) did similarly as the first observational results become available. Generally, the observed surface density of submm galaxies was underpredicted. Prior to the recognition that an isotropic signal in the far-IR COBE-FIRAS data was an extragalactic background (Puget et al., 1996) and not Zodiacal emission (Mather et al., 1994), this could be accounted for by the use of this unduly restrictive limit on the intensity of the submm background spectrum. Once submm count data became available after SCUBA was commissioned, it was possible to take either a more empirical or a more theoretical view of the consequences for dusty galaxy evolution.

On the empirical side, forms of evolution of the low-redshift luminosity function of dusty galaxies (Saunders et al., 1990; Soifer and Neugebauer, 1991) that were required to fit the count and background data could be determined (Malkan and Stecker, 1998, 2001; Blain et al., 1999b; Tan et al., 1999; Pearson, 2001; Rowan-Robinson, 2001). At redshifts less than about unity, this is done by requiring that the predicted counts of low-redshift IRAS and moderate-redshift $I S O$ galaxies are in reasonable agreement with observations. At greater redshifts, where the form of evolution is constrained by submm count and background data, there is significant degeneracy in the models: strong evolution could proceed all the way out to a relatively low cutoff redshift $(z \sim 2-3)$, or the strong evolution could terminate at a lower redshift $z \sim 1$, followed by a tail of either non-evolving or declining luminosity den- 
sity out to greater redshifts ( $z \geq 5$ ) (see Fig. 9 of Blain et al., 1999b). This degeneracy occurs because the far-IR background radiation (like almost all backgrounds) is generated predominantly at $z \sim 1$, while submm galaxies can contribute to the counts equally at almost any redshift $1<z<10$. It can be broken by determining a redshift distribution of submm galaxies, which would be very different in the two cases. The form of evolution that is consistent with the latest observational constraints and radio-derived redshift information for submm-selected galaxies (Smail et al., 2002) is shown by the thick solid and dashed lines in Fig. 21.

Note that the assumptions that underlie these derivations are not yet all verified by observations. It is unclear whether all high-redshift dusty galaxies detected in submm surveys have similar SEDs. It is possible that the properties of the dust grains in galaxies evolve with redshift, leading to a systematic modification to the temperature or emissivity index. It is reasonable to expect the dust-to-gas ratio in the highest redshift galaxies to be lower than in low-redshift galaxies, as less enrichment has taken place. However, note that enrichment proceeds very rapidly once intense star formation activity is underway. Even the very first regions of intense star formation could thus be readily visible in the submm, despite the global metallicity being extremely low. While it seems unlikely, based on a handful of observations (Fig. 2), it is certainly possible that a population of dusty galaxies with a significantly different SED is missing from current calculations (Blain and Phillips, 2002).

A more theoretically-motivated approach, based on making assumptions about the astrophysical processes at work in galaxy evolution and then predicting the observational consequences, has rightly become popular in recent years. These 'semi-analytic' models, which were generally developed to explain optical and near-IR observations, take a representative set of dark-matter halos that evolve and merge over cosmic time, from the results of N-body simulations, and determine their star-formation histories and appearance using a set of recipes for star-formation and feedback (White and Frenk, 1991; Kauffmann and White, 1993; Cole et al., 1994, 2000; Guiderdoni et al., 1998; Granato et al., 2000, 2001; Baugh et al., 2001; Benson et al., 2001; Somerville et al., 2001). Unfortunately, at present there is insufficient information from submm observations to justify a model that contains more than a handful of uncertain parameters, and so it is difficult to exploit the full machinery of semi-analytic models to explain the submm observations. Despite the free parameters available, semi-analytic models have had limited success in accounting for the observed population of high-redshift submm galaxies, without adding in an extra population of more luminous galaxies to the standard prescription (Guiderdoni et al., 1998), or breaking away from their traditional reliance on a universal initial mass function (IMF). As more information becomes available, then the full capabilities of the semi-analytic models can hopefully be applied to address dusty galaxy evolution. 


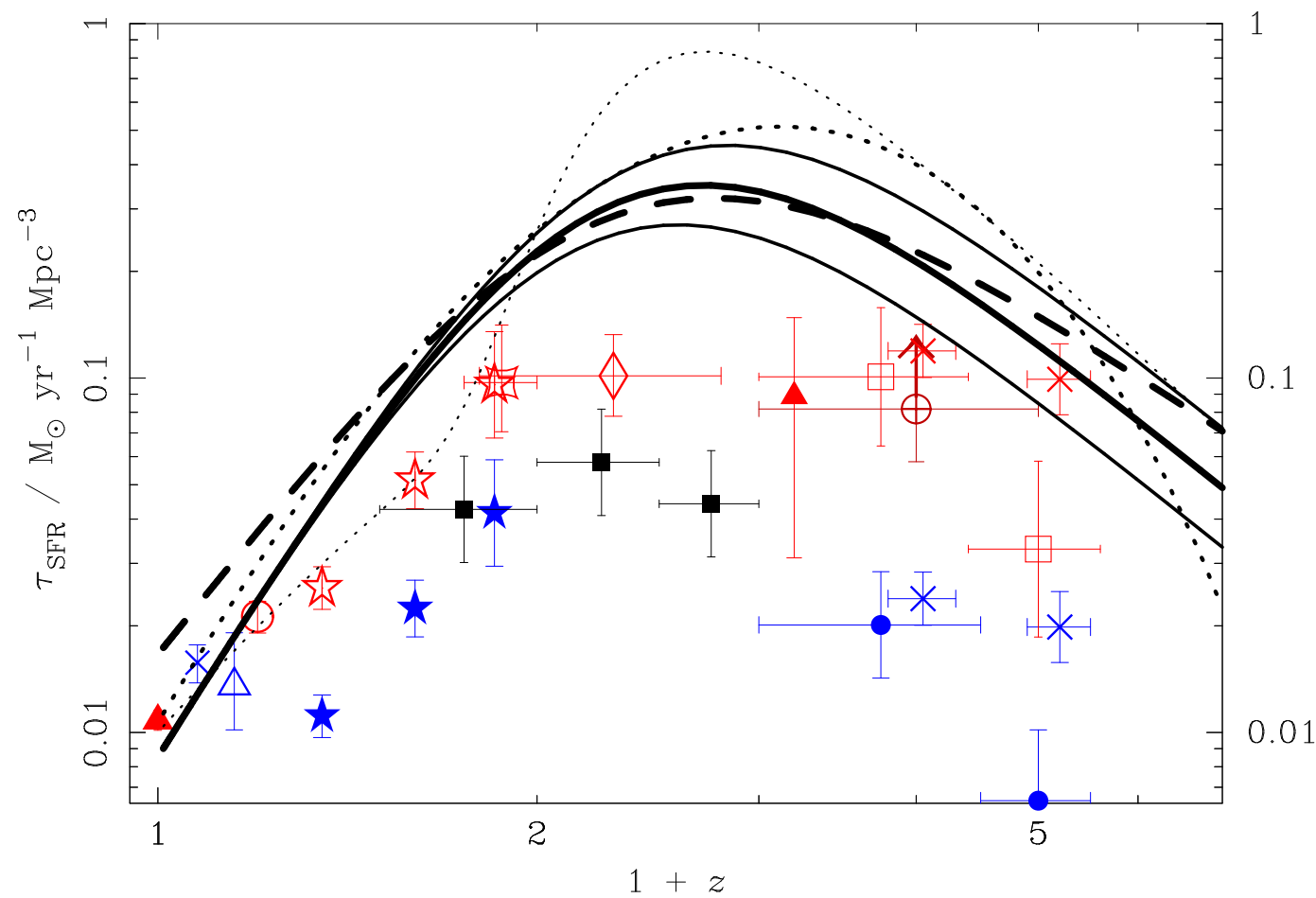

Fig. 21. The history of energy generation in the Universe, parameterized as a star-formation rate per unit comoving volume. The absolute normalization of the curves depends on the assumed stellar IMF and the fraction of the dust-enshrouded luminosity of galaxies that is generated by AGN. The points show results derived from a large number of optical and near-IR studies, for which detailed references can be found in Blain et al. (1999b) and Smail et al. (2002). The most important results are from Lilly et al. (1996; filled stars) and Steidel et al. (1999; high-redshift diagonal crosses). The up-pointing arrow comes from the submm-based estimate of Hughes et al. (1998). An important new measurement of the extinction-free low-redshift star-formation rate from radio data, that is not plotted, has been obtained by Yun et al. (2001): $0.015 \pm 0.005 \mathrm{M}_{\odot} \mathrm{yr}^{-1}$. The thick solid and dashed lines represent the current best fits to far-IR and submm data in a simple luminosity evolution model and a hierarchical model of luminous merging galaxies respectively, as updated to reflect additional data and a currently favored non-zero- $\Lambda$ cosmology. The thinner solid lines show the approximate envelope of $68 \%$ uncertainty in the results of the luminosity evolution model. The thin and thick dotted lines represent the best-fitting results obtained in the original derivations (Blain et al., 1999b, c).

In a blend of these approaches, assuming that the SCUBA galaxies are all associated with merging galaxies (Ivison et al., 1998a, 2001; Figs. 14 and 18), and yet not being sure of the physical processes by which the mergers generate the luminosity we observe, Blain et al. (1999c) used a minimally-parametrized semi-analytic model to investigate the change in the properties of merging galaxies required to reproduce the submm and far-IR counts and backgrounds: see also Jameson (2000) and Longair (2000). The observed background spectrum can only be reproduced for strong evolution of the total luminosity den- 
sity out to redshift $z \simeq 1$, by a factor of about 20 (see Fig. 21). In addition, the lifetime of the luminous phase associated with mergers, and thus the mass-tolight ratio also had to be reduced by a large factor at high redshift in order to reproduce the observed submm counts. The physical reason for this change must be an increased efficiency of star formation during starburst activity or of AGN fueling at increasing redshifts; both make sense in light of the greater gas densities expected at high redshifts. The model has the advantage of being able to reproduce the faint optical counts, if blue LBGs are also associated with merging galaxies. The submm galaxies release about four times more energy in total than the LBGs, and do so over a period of time during a merger that is about ten times shorter. It is likely that the total baryonic mass and geometry of the merging galaxies also play important roles in determining the details of star-formation activity or AGN fueling during a merger.

When reviewing the predictions and results of any model, note that it is easy to produce a model that can account for the far-IR-submm background radiation intensity; more difficult to account for the submm counts; and more difficult again to reproduce a plausible redshift distribution.

\subsection{Observational tests of models}

The key observational test of models of submm-wave galaxy formation is the redshift distribution, which is know in outline from radio-submm observations (Smail et al., 2000). Determining the redshift distribution is a key goal of extensive ongoing follow-up observations, but the process has proved to be difficult and time-consuming, as documented extensively by Smail et al. (2002). The crucial problems are the faintness of the counterparts, combined with the relatively poor positional accuracy of the centroids of the submm galaxies, which are unresolved due to the coarse spatial resolution of existing submm images.

Detailed measurements of the counts of galaxies at both brighter and fainter flux densities than those shown in Fig. 9 would also constrain models. However, determining the bright counts requires a large-area survey, which is likely to be relatively inefficient (Fig. 23), while determining the faint counts requires greater angular resolution than can be provided by the telescopes used to make existing surveys, to avoid source confusion. The very bright counts will certainly be probed directly towards the end of the decade by the Planck Surveyor all-sky survey at a resolution of 5 arcmin, and sooner by large-area surveys using forthcoming large-format $\mathrm{mm} / \mathrm{submm}$-wave bolometer arrays on ground-based telescopes, including BOLOCAM (Glenn et al., 1998) and SCUBA-II. Limits on the bright submm-wave counts can be imposed from the number of candidate point sources that can be found in large-area submm 
maps of Galactic fields (Pierce-Price et al., 2001; Barnard et al., 2002). The faint counts will ultimately be determined directly using the SMA, CARMA and ALMA interferometers.

The results of deep $I S O$ surveys have been regularly cited as a useful constraint on galaxy evolution (Rowan-Robinson et al., 1997; Xu, 2000; Chary and Elbaz, 2001). This is certainly true out to $z \simeq 1$. However, when estimating a total luminosity density from $15-\mu \mathrm{m}$ data, it is vital that the correct SED is used to extrapolate to longer wavelengths, as it is easy to overestimate the amount of luminosity associated with a $15-\mu \mathrm{m}$ source by assuming a mid-IR SED that is too steep. For example, compare the inferred luminosity density results at redshifts $z \simeq 0.7$ quoted by Rowan-Robinson et al. (1997) and Flores et al. (1999). The results differ by a factor of 5; Flores et al. (1999) obtain the lower result by using radio observations to constrain the total luminosity of the galaxies detected at $15 \mu \mathrm{m}$. Extrapolating mid-IR data towards the peak of the SED at longer wavelengths is more difficult than extrapolating submm observations to fix the position of the peak of the SED that lies at shorter wavelengths. This is both because the form of the SED is intrinsically simpler on the long-wavelength side of the peak, and because the well-determined spectrum of the far-IR background radiation can be used to constrain the luminosity-averaged dust temperature of the submm galaxies. Mid-IR observations with SIRTF after 2002 will provide much more information about the SEDs and evolution of dusty galaxies to redshifts $z \simeq 2$.

In Fig. 22 we show the deep 15- $\mu$ m counts predicted by models designed to account for the submm data (Blain et al., 1999b, c), updated to the current data and cosmology. If the mid-IR SED is chosen appropriately, then the fit is quite acceptable. Including PAH emission features or varying the mid-IR SED index $\alpha$ has relatively little effect on the result. The same approach can be used to estimate the deep cm-wave radio counts. If we assume just the form of the radio-far-IR correlation (Condon, 1992), without any fine tuning, and a radio SED of the form $f_{\nu} \propto \nu^{-0.6}$, then the predicted $8.4-\mathrm{GHz}$ counts brighter than $10 \mu \mathrm{Jy}$, based on the submm-based models are 1.05 and 0.98 arcmin $^{-2}$ respectively; the corresponding power-law indices of the count function $N(>S) \propto S^{\alpha}$ are $\alpha=-1.4$ and -1.3 respectively. The results in both models match the observed 8.4-GHz 10- $\mu \mathrm{Jy}$ count of $1.01 \pm 0.14 \mathrm{arcmin}^{-2}$ with $\alpha=-1.25 \pm 0.2$ (Partridge et al., 1997). The reasonable agreement between the predictions of the models, which are constrained only by observations in the submm and far-IR, and the observed deep mid-IR and radio counts confirms that the models are reliable. The source confusion estimates shown in Fig. 13, which are based on the same models, should thus be reliable over a wide wavelength range from about $10 \mathrm{~cm}$ to $10 \mu \mathrm{m}$. 


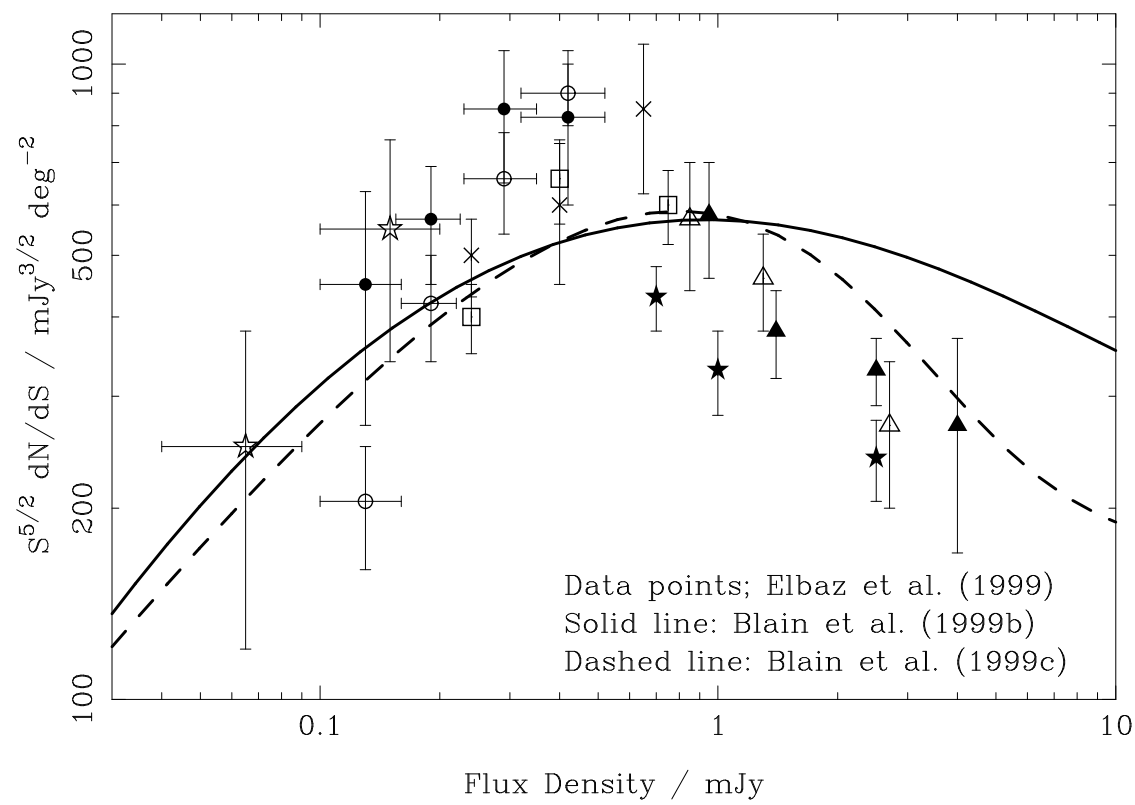

Fig. 22. A summary of observed (Elbaz et al., 1999) and predicted (Blain et al., $1999 \mathrm{~b}, \mathrm{c})$ differential counts of galaxies in the 15- $\mu \mathrm{m}$ ISO band. The model predictions assume only a power-law SED in the mid-IR, with $f_{\nu} \propto \nu^{-1.95}$ : no fine-tuning with PAH features in the SED is included. The hierarchical model (Blain et al., 1999c) provides a better fit to the data, but both agree reasonably well with the observations. Both models asymptote to the same count at greater flux densities. Note that the relative form of the model counts reflects that seen in Fig. 9, with the hierarchical model having the steepest rise.

\subsection{Modeling the detailed astrophysics of the submm galaxies}

It is not possible to separate the modeling of the evolution of the population of submm galaxies fully from studies of the nature of the galaxies themselves. Their luminosities and masses (see Frayer et al., 1999) demand that the submm-luminous phase be short-lived as compared with the age of the Universe. The observational information for most of the submm galaxies is insufficient to be confident that their nature is understood at present. Of the galaxies with reliable counterparts, there are three bright Class-2 galaxies (Soucail et al., 1999; Ivison et al., 1998a, 2000a, 2001; Vernet and Cimatti, 2001) with optical redshifts and CO detections (Frayer et al., 1998, 1999; Kneib et al., 2002), and a total of four Class-1 galaxies (Smail et al., 1999; Bertoldi et al., 2000; Gear et al., 2000; Lutz et al. 2001), which are known to be either very red galaxies or formal EROs. Other submm-selected galaxies with accurate positions from radio observations (Smail et al., 2000) or mmwave interferometry (Downes et al., 1999b; Bertoldi et al., 2000; Frayer et al., 2000; Dannerbauer et al., 2002) remain enigmatic. All that can be said about these galaxies is that they all appear to have thermal dust spectra, are all very faint at optical wavelengths, and most also appear to be very faint at near-IR wavelengths. 
The Class-2 galaxies are all clearly undergoing mergers or interactions. Much less is known about the morphology of the faint, but typically extended Class-1 galaxies (Figs. 16, 17 and 20). It is certainly possible that they too are involved in interactions, which appear to trigger the dramatic luminosity of almost all the low-redshift ULIRGs (Sanders and Mirabel, 1996). Programs of ultradeep near-IR imaging on 10-m-class telescopes should soon test this idea.

Hydrodynamical simulations of gas-rich mergers by Mihos and Hernquist (1996), Bekki et al. (1999) and Mihos (2000) show the formation of very dense concentrations of gas, which could be associated with short-lived, very-intense bursts of star formation. However, at present it is not possible to simulate a representative sample of mergers with the range of geometries likely to be encountered, the necessary time resolution, and a sufficiently accurate treatment of the detailed astrophysics of star-formation to make a reliable connection between the limited observations and the underlying galaxy properties. The spatial extent of the three bright Class- 2 galaxies in the optical waveband appears to be considerably greater than that of most low-redshift ULIRGs. It is thus difficult to be sure that simulations of well-studied low-redshift ULIRGs adequately represent the properties of the high-redshift submm galaxies. Note, however, that the precise spatial relationship between the optical and submm emission in these objects is still unclear (Ivison et al., 2001); the submm emission could be more compact than the optical galaxy.

Larger submm galaxy samples will be available over the next few years, boosting the likelihood that examples of the full range of submm galaxies will be available to be studied in detail. More sensitive observations of the properties of the known galaxies will also improve our knowledge of their astrophysics. One key question is the relationship of the submm galaxies to the formation of elliptical galaxies (Lilly et al., 1999). Whether the bulk of submm galaxies are high-redshift low-angular-momentum gas clouds, forming elliptical galaxies in a single episode by a 'monolithic collapse' (Eggen et al., 1962), as advocated by for example Archibald et al. (2002), or galaxies observed during one of a series of repeated mergers of gas-rich, but pre-existing galaxy sub-units, likely to take place at relatively lower redshifts, as discussed by Sanders (2001), and which might ultimately yield elliptical merger remnants, is an important question that future follow-up observations will address. Existing observations of extended and disturbed counterparts to submm galaxies (Ivison et al., 1998a, 2001; Lutz et al., 2001) tend to favor the second explanation, in which welldefined pre-existing stellar systems merge. However, in either scenario it is likely that the bulk of the stellar population in the resulting galaxies form during the submm-luminous phase. 


\subsection{The global evolution of dust-enshrouded galaxies}

Fig. 21 summarizes the current state of knowledge of the strong evolution of the comoving luminosity density contributed by luminous far-IR galaxies, whose emission is redshifted into the submm (Blain et al., 1999b, c; Smail et al., 2002). The derivation of these results was discussed briefly above, and is explained in much more detail in these papers. The results of both the luminosity evolution and hierarchical models, which both include strong luminosity evolution of the population of low-redshift IRAS galaxies out to high redshifts, are fully compatible with the redshift information available for submm galaxy samples. This would not be the case if the redshift evolution of the luminosity density was markedly different from the forms shown in Fig. 21. For example, if the luminosity density of submm galaxies were to match rather than exceed the value denoted by the datapoints at $z \simeq 1$ in Fig. 21 , and remain at the same high level out to $z \simeq 10$, then the submm-wave spectrum of the cosmic background radiation (Fig. 11) would tend to be too flat, the intensity of the far-IR background radiation would fall short of the observed level, and the predicted redshift distribution of submm galaxies would be biased strongly to the highest redshifts, which at present seems not to be the case (Smail et al., 2002). The determination of complete redshift distributions for existing samples of submm galaxies, and of more redshifts for individual very luminous galaxies drawn from large, future submm-selected galaxy catalogs, will ultimately allow the evolution of distant dusty galaxies to be traced in detail.

\section{Gravitational lensing in the submm waveband}

The first submm-wave surveys for distant galaxies exploited both the weak to moderate gravitational lensing magnification, by about a factor of 2-3, experienced throughout the inner few square arcminutes of rich foreground cluster of galaxies at a moderate redshift in the range $z \simeq 0.2-0.4$ (Blain, 1997) and the greater magnification produced along critical lines for much smaller areas of the background sky. A 5-arcmin ${ }^{2}$ SCUBA field centered on a moderate redshift cluster includes both these regions, enhancing the flux density from all high-redshift background galaxies (Smail et al., 1997). More, and in some cases deeper, SCUBA images of clusters have been taken (Smail et al., 2002; Chapman et al., 2002a; Cowie et al., 2002), especially in Abell 2218, where a multiply-imaged source has been detected (van der Werf and Kraiberg Knudsen, 2001). Whether the magnification acts to increase the surface density of background galaxies on the sky depends on the form of their counts. Lensing by both galaxies and clusters could have significant applications in future submm surveys, especially those sampling the steep counts of bright submm galaxies in wide fields, including the all-sky survey from Planck Surveyor (Blain, 1998), 
and surveys using BLAST, Herschel-SPIRE and SCUBA-II (Tables 1 and 2) covering many tens of square degrees.

\subsection{Magnification bias}

Because surface brightness is conserved by all gravitational lenses, the net effect of magnifying a population of background galaxies depends on the slope of the counts $\mathrm{d} N(>S) / \mathrm{d} S$, where $N(>S)$ is the number of galaxies per unit area on the sky brighter than $S$ (Schneider et al., 1992). Subject to a magnification $\mu$, the count becomes $\left[1 / \mu^{2}\right] \mathrm{d} N[>(S / \mu)] / \mathrm{d} S$. For a power-law count with $\mathrm{d} N(>S) / \mathrm{d} S \propto S^{\alpha}$, a value of $\alpha<-2$ corresponds to an increase in surface density if $\mu>1$. This threshold value corresponds to a slope of -1 for the integral counts $N(>S)$ shown in Fig. 9. Note that for a uniform non-evolving population of galaxies $\alpha=-2.5$.

As shown in Fig. 9, submm-wave counts are expected to be steep, and to change slope sharply at mJy flux density levels, as compared with deep optical or radio counts. The significant changes in the count slope are particularly unusual, and not found in any other waveband. As a result, the magnification bias can be large, increasing the number of detectable bright galaxies (Blain, 1996, 1997), and providing a way to investigate very faint counts by comparing lensed and unlensed fields; for example in the innermost regions of clusters of galaxies (Blain, 2002).

That a significant magnification bias can be exploited using relatively weak lensing by clusters of galaxies can be seen by comparing the number of 1020-mJy 850- $\mu \mathrm{m}$ galaxies detected in the SCUBA Lens Survey (Smail et al., 1997, 2002), and in the larger field of the unlensed 8-mJy survey (Scott et al., 2002). The ratio is about $3: 1$, showing a clear positive magnification bias, and indicating that if the $850-\mu \mathrm{m}$ counts at flux densities greater than $10 \mathrm{mJy}$ are represented by a power-law, then the index $\alpha<-2$.

\subsection{Conditions for exploiting submm lensing by galaxies}

The key advantage of observing background galaxies that are gravitationally lensed by foreground mass concentrations in the submm waveband is that the K correction (Fig. 4) acts to brighten the distant background lensed galaxy as compared with the lens. This is already very familiar from surveys of lensed radio AGN (Rusin, 2001), and is illustrated clearly in Fig. 1, in which only the central cD galaxy in the lensing cluster shows any significant submm emission.

In SCUBA cluster lens surveys, both the image separations, and the extent 
of the high-magnification regions are of order 1 arcmin, a scale which is well matched both to the 15-arcsec resolution of the JCMT and to the 2.5-arcmin field of view of SCUBA. The magnification ensures that a significantly greater fraction of the submm-wave background radiation intensity is thus resolved into detectable galaxies in surveys in the fields of gravitational lensing clusters than in even the deepest blank-field surveys (Blain et al., 1999a). However, for background sources lensed by galaxies rather than clusters, the relevant image separations and the extent of the high-magnification region are only of order 1 arcsec, and so cannot be resolved using any single-antenna telescope. High-resolution submm observations are required to disentangle lensed and unlensed galaxies; this capability will be provided by ALMA (Blain, 2002), while pilot studies of should be possible using the CARMA, SMA and IRAM PdBI interferometers. The most luminous lensed sources can already be resolved into multiple images using the IRAM mm-wave interferometer (Alloin et al., 1997).

The only caveat for exploiting galaxy-scale lensing is that the source size must be small as compared with the area of sky behind the lens that is strongly magnified. The intense far-IR and submm emission from low-redshift ULIRGs is typically very compact (several hundred pc across; Downes and Solomon, 1998), and would easily meet this condition; however, there are indications that the dust emission from at least some luminous high-redshift submm galaxies could extend over scales greater than 10-kpc (Papadopoulos et al., 2001; Chapman et al., 2001a; Lutz et al., 2001; Isaak et al., 2002; Ivison et al., 2001). The whole area of sky covered by these galaxies would not then be lensed efficiently by an intervening galaxy, although bright knots of emission within them could still be magnified by large factors. This concern about lensing efficiency and the angular size of distant submm galaxies does not apply to lensing by much larger clusters of galaxies, which will always be effective.

\subsection{Prospects for the lensing studies in the future}

Larger area surveys for brighter samples of luminous dusty galaxies using the array of forthcoming ground-based, air- and space-borne instruments, including BOLOCAM, BLAST, SOFIA, SCUBA-II, SIRTF, Herschel and Planck Surveyor (see Table 2), should be subject to an enhanced magnification bias. High-resolution follow-up observations using ALMA should then yield a large sample of strongly magnified high-redshift lensed systems to complement the systematically selected CLASS sample of gravitational lensed AGN identified at radio wavelengths (Rusin, 2001). These surveys will not be subject to any extinction bias due to absorption by dust in the lensing galaxies, and should yield a very complete and reliable catalog of up to several thousand lenses (Blain, 1998). 


\section{$7 \quad$ Future developments in submm cosmology}

During the last 4 years, the first steps have been taken towards investigating the Universe using direct submm-wave surveys. The technologies of the class of detectors that made these initial surveys possible are still developing rapidly. Many instrumentation projects are underway, which will allow us to increase the sizes of samples of distant submm galaxies, and to study known examples in more detail; some of their key features are outlined in Tables 1-3.

\subsection{New technologies for instrumentation}

A key technology under development is for bolometers with superconducting temperature-sensitive elements, including transition-edge sensors (TESs). These are much more stable than the semiconducting thermistors used in existing systems, and so can be read out using multiplexed, and therefore much simpler, cold electronics. Another advantage of TES devices is that they require no bias current, and so need fewer heat-conducting, difficult-to-assemble connections to each device. The prototype Fabry-Perot spectroscopic device FIBRE, which uses TES bolometers (Benford et al., 2001), was tested successfully at the CSO in May 2001.

TES devices offer the prospect of increasing the size of the arrays of detector elements in mm/submm-wave cameras from of order 100 to of order $10^{4-5}$, providing much larger fields of view. Filled-array detector devices using conventional semiconducting bolometers are being demonstrated in the SHARC-II and HAWC cameras for the CSO and SOFIA, while the SCUBA-II camera has a goal of at least a $8 \times 8$ arcmin field of view-about 25 times greater than the field of view of SCUBA - is under development in Edinburgh and is expected to integrate large arrays and superconducting bolometers. SCUBA-II will supplement its much wider field of view with an enhanced point source sensitivity: the same galaxies should be detectable about 8 and 4 times faster using SCUBA-II as compared with SCUBA at wavelengths of 850 and $450 \mu \mathrm{m}$ respectively: see Table 1 . A $10-\mathrm{m}$ telescope operating at $850-\mu \mathrm{m}$ with a $10^{5}$ element detector would have a square field of view about $1 \mathrm{deg}$ on a side, at a

Nyquist-sampled plate scale: much larger than the 5-arcmin ${ }^{2}$ fields of view of SCUBA and MAMBO. With such large fields of view, it is not unreasonable to survey most of the sky down to the confusion limit of a 10-30-m telescope in an observing campaign lasting for several years.

By combining large numbers of bolometer detectors with dispersive mm-wave optics (Glenn, 2001), it should be possible to obtain low-resolution mm-wave spectra of galaxies over a very wide band, perhaps $100 \mathrm{GHz}$, to search for CO 
and atomic fine-structure line emission from high-redshift submm galaxies detected in continuum surveys, while simultaneously carrying out unbiased surveys for line-emitting galaxies within the field of view (Blain et al., 2000b). Development of several such systems is underway.

Phase-sensitive heterodyne submm detectors are already very efficient; however, only small numbers of these detectors can currently be fabricated into an array. Their strength is in very high resolution submm-wave spectroscopy, and as sensitive coherent detectors in existing mm-wave interferometers. They will be fitted to the forthcoming SMA and CARMA, and will be be exploited to the full with the large collecting area of the ALMA array.

Sensitive arrays of mid- and far-IR detectors should soon be flying, both in space aboard SIRTF and ASTRO-F, and in the upper atmosphere, on balloons such as BLAST, and aboard SOFIA. Limits to the continuum flux densities of the most luminous high-redshift galaxies derived using these facilities, measured close to the peak of their SED (see Fig. 2), will provide valuable information about their properties. Spectrographs, both aboard these facilities and on ground-based telescopes, will provide detections of and sensitive limits to the line radiation from the same objects, providing redshift information and astrophysical diagnostics.

\subsection{New telescopes}

At present, a wide range of submm-wave telescopes are available. Singleantenna telescopes include the 10.4-m CSO on Mauna Kea, the 10-m Heinrich Hertz Telescope (HHT) on Mount Graham, the 15-m JCMT on Mauna Kea, the 15-m SEST at La Silla in Chile, the 30-m IRAM telescope in Spain, and the 45-m antenna at Nobeyama in Japan. The Large Millimeter Telescope (LMT), a 50-m mm-wave telescope is under construction on a 5000-m peak near Puebla in Mexico, and it is hoped that the 100-m Green Bank Telescope (GBT) in West Virginia can operate at $90 \mathrm{GHz} / 3 \mathrm{~mm}$ during the winter. New single-antenna telescopes with large survey cameras have been proposed for the excellent submm observing sites at the South Pole and the ALMA site in Chile. The Planck Surveyor CMB imaging mission will generate an all-sky map in the submm at a resolution of 5 arcmin, and the 3.5-m Herschel space telescope will carry out pointed submm imaging and spectroscopic observations of known galaxies, and carry out deep confusion-limited cosmological surveys over fields several hundred square degrees in size. Cameras exploiting the $2.5-\mathrm{m}$ telescope aboard SOFIA and BLAST and other dedicated ultra-long-duration balloon instruments will allow far-IR and submm-wave observations from the upper atmosphere. 
Existing mm-wave interferometers include the $6 \times 15$-m IRAM PdBI, the $6 \times$ 10.4-m OVRO MMA, the $10 \times 6$-m BIMA array at Hat Creek in California and the $6 \times 10-\mathrm{m}$ Nobeyama Millimeter Array. The $8 \times 6-\mathrm{m}$ SMA is under construction on Mauna Kea, the first imaging submm-wave interferometer, while it is planned to combine 9 of the BIMA antennas with the OVRO MMA at a high site in the Inyo Mountains east of Owens Valley in California to form CARMA. The international $64 \times 12-\mathrm{m}$ ALMA submm interferometer array in Chile will provide a tremendous increase in the capability of submm-wave spectral line and continuum imaging, providing 10- to 30- $\mu$ arcsec resolution, and detailed images of even the most distant galaxies. The most luminous submm galaxies so far discovered, with $850-\mu \mathrm{m}$ flux densities of about $25 \mathrm{mJy}$, could be detected at a 10- $\sigma$ significance by ALMA in about a second. Its excellent sensitivity and wide $8-\mathrm{GHz}$ instantaneous bandwidth will allow a significant fraction of the galaxies detected in deep surveys to be detected simultaneously in the continuum and $\mathrm{CO}$ rotation lines, providing direct and exact redshifts. As the redshifted ladder of CO lines are separated by 115/(1+ z) $\mathrm{GHz}$, about $25 \%$ of galaxies at $z \simeq 2.5$ will have a CO line lying within the 8-GHz-wide ALMA band (Blain et al., 2000b).

\subsection{Future capabilities and progress}

The enhanced capabilities of this array of new facilities is illustrated in Fig. 23. The rate at which galaxies can be detected is likely to grow dramatically from a few per day at present to many hundreds per hour. Note that the various instruments operate at different wavelengths, and so each is most sensitive to galaxies at different redshifts and with different luminosities. However, sample sizes are certain to increase dramatically, especially when the $10^{4}-10^{5}$ galaxies that will be detected in the Planck Surveyor all-sky survey are taken into account.

Multiwavelength follow-up observations of all these new submm galaxies are likely to remain a time-consuming challenge. However, the likely availability of 30-m-aperture ground-based optical/near-IR telescopes in the next decades, and the extremely deep imaging capability of NGST, should help us to study a complete sample of submm galaxies down to luminosities that are only a fraction of $L^{*}$.

\section{Summary: key questions and targets for the future}

The first generation of extragalactic submm-wave surveys have provided an important complement to more traditional optical and radio searches for dis- 
Table 1

Wavelengths $\lambda$, sensitivities (as noise equivalent flux density-NEFD), fields of view (FOV), and confusion limits due to galaxies and the ISM (in brackets) for existing and future ground-based and airborne instruments.

\begin{tabular}{lllll}
\hline Name & $\lambda$ & $\begin{array}{l}\text { NEFD } \\
(\mu \mathrm{mJy} / \sqrt{H z})\end{array}$ & $\begin{array}{l}\text { FOV } \\
\left(\operatorname{arcmin}^{2}\right)\end{array}$ & $\begin{array}{l}\text { Confusion } \\
(\mathrm{mJy})\end{array}$ \\
\hline SCUBA & 850 & 80 & 1.7 & $0.12\left(9 \times 10^{-4}\right)$ \\
& 450 & 160 & 1.7 & $0.053\left(3 \times 10^{-3}\right)$ \\
MAMBO $^{1,2}$ & 1250 & 95 & 1.0 & $0.05\left(7 \times 10^{-5}\right)$ \\
SCUBA-II & 850 & 28 & 64 & $0.12\left(9 \times 10^{-4}\right)$ \\
& 450 & 90 & 64 & $0.053\left(3 \times 10^{-3}\right)$ \\
HAWC-SOFIA & 200 & 408 & 9.0 & $1.2(0.30)$ \\
BOLOCAM-CSO & 1100 & 42 & 44 & $0.32\left(2 \times 10^{-3}\right)$ \\
BOLOCAM-LMT & 1100 & 2.8 & 2.5 & $6 \times 10^{-3}\left(4 \times 10^{-5}\right)$ \\
BLAST & 750 & 115 & $\simeq 10$ & $3.9(0.25)$ \\
& 450 & 130 & $\simeq 10$ & $6.8(0.7)$ \\
SKA & 300 & 150 & $\simeq 10$ & $7.8(1.1)$ \\
SMA & 850 & 170 & 0.2 & $<10^{-7}\left(<10^{-6}\right)$ \\
ALMA & 450 & 1700 & 0.05 & $<10^{-8}\left(<10^{-4}\right)$ \\
& 870 & 1.9 & 0.050 & $<10^{-7}\left(<10^{-6}\right)$ \\
Antended VLA & $20.5 \mathrm{~cm}$ & 0.40 & 0.013 & $<10^{-8}\left(<10^{-4}\right)$ \\
& $20.5 \mathrm{~cm}$ & $\sim 10^{-2}$ & 700 & $\sim 0(\sim 0)$ \\
& 450 & 11 & $\sim 0(\sim 0)$ \\
\hline
\end{tabular}

An estimate of the speed of a survey down to a chosen depth can be obtained by multiplying the FOV by the the square of the NEFD value. The approximate extragalactic confusion noise values are the flux density at which there is one brighter source per beam (Blain et al., 1998; Fig. 13). This corresponds approximately to the width of peak in the non-Gaussian confusion noise distribution (see Fig. 12). The expected ISM confusion noise (in brackets) is calculated for a $100-\mu \mathrm{m}$ surface brightness $B_{0}=1 \mathrm{MJy} \mathrm{sr}^{-1}$ (Helou and Beichman, 1990), and scales as $B_{0}^{1.5}$. Other instruments under development, which have not published detailed performance estimates include the $350-\mu \mathrm{m}$ SHARC-II camera for the CSO. The FOV and NEFD values are chosen to provide the correct results for making a fully-sampled image of the sky, not measuring the flux from a single galaxy. Updated from Table 1 in Blain (1999b). Relevant references are listed in Table 3. TBD: to be decided.

1 Note that the FOV of MAMBO is expanded by a factor of 3 for the winter of 2001/2002, with a 117-bolometer detector array. ${ }^{2}$ A similar device SIMBA is being commissioned at SEST. 


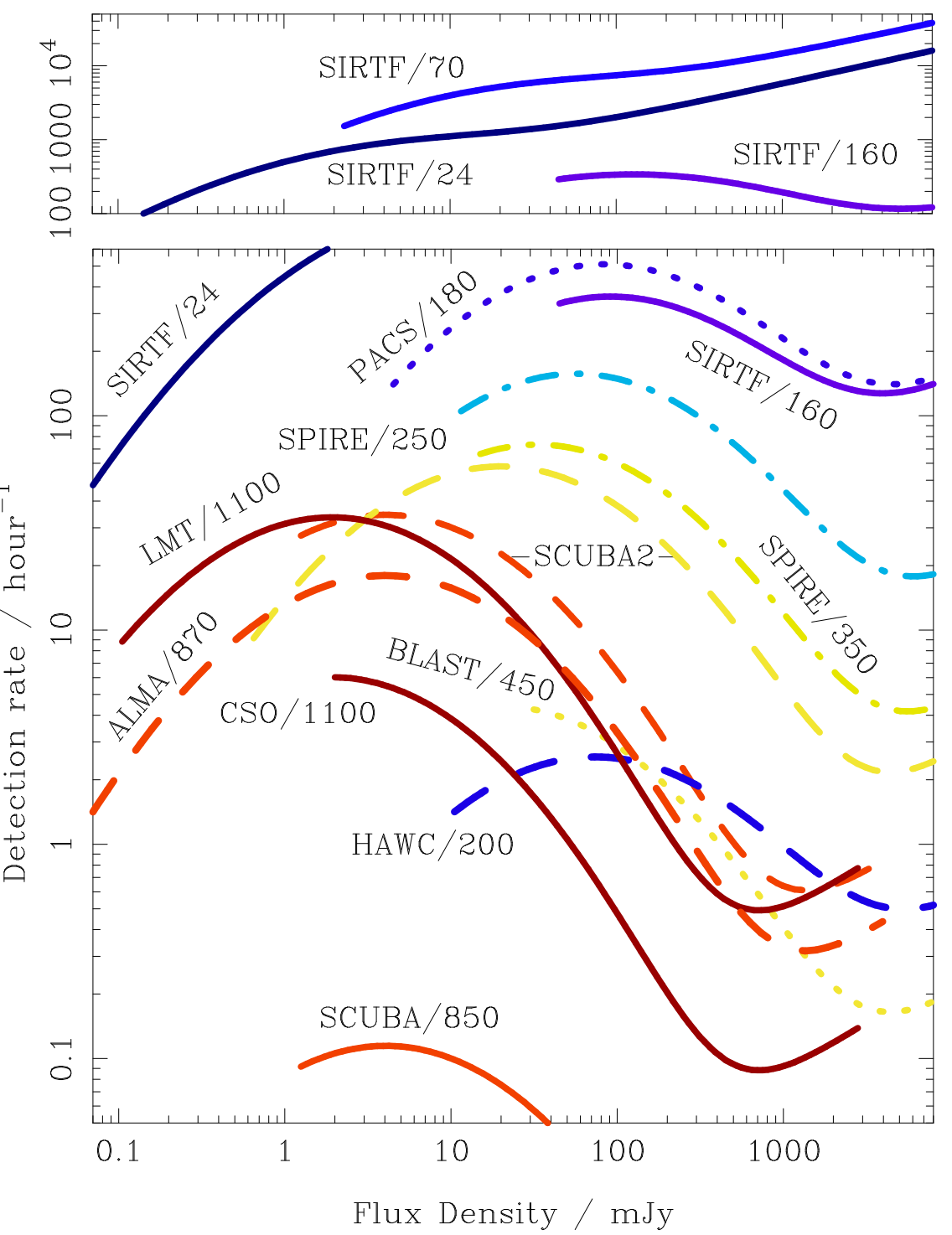

Fig. 23. The detection rates expected in a variety of forthcoming $\mathrm{mm}$, submm and far-IR surveys. The names and wavelengths in microns of the relevant instruments are listed: see Tables 1 and 2. The higher- and lower-peaking SCUBA-II curves correspond to wavelengths of 450 and $850 \mu \mathrm{m}$ respectively. References to instrument performance for these calculations (Blain and Longair, 1996) can be found in Table 3. The surface density of galaxies assumed follows the models of Blain et al. (1999b): see Figs. 9 and 10. Curves stop on the right if the surface density is expected to fall below a single galaxy on the whole sky. Curves stop at the left when a relatively optimistic definition of the $5 \sigma$ confusion noise level for detection is reached (see Fig. 13).

tant galaxies, and discovered a cosmologically significant population of veryluminous, high-redshift dusty galaxies.

We have found that is very hard to study a complete sample of submm galaxies at other wavelengths (Smail et al., 2002). The similar experience of other 
Table 2

The equivalent to Table 1 for space-borne instrumentation.

\begin{tabular}{lllll}
\hline Name & $\lambda$ & $\begin{array}{l}\text { NEFD } \\
(\mathrm{mJy} / \sqrt{H z})\end{array}$ & $\begin{array}{l}\text { FOV } \\
\left(\operatorname{arcmin}^{2}\right)\end{array}$ & $\begin{array}{l}\text { Confusion } \\
(\mathrm{mJy})\end{array}$ \\
\hline Herschel-SPIRE & 500 & 114 & 40 & $2.9(0.16)$ \\
& 350 & 90 & 40 & $2.6(0.12)$ \\
& 250 & 84 & 40 & $1.6(0.24)$ \\
Herschel-PACS & 170 & 24 & 6.1 & $0.80(0.16)$ \\
& 90 & 24 & 6.1 & $0.03(0.01)$ \\
SIRTF-MIPS & 160 & 18 & 2.5 & $6.6(3.1)$ \\
& 70 & 4.5 & 25 & $0.28(0.07)$ \\
SIRTF-IRAC & 8.0 & 0.15 & 25 & $6 \times 10^{-4}\left(2 \times 10^{-4}\right)$ \\
Planck Surveyor & 350 & 26 & 26 & $8 \times 10^{-2}\left(\sim 10^{-6}\right)$ \\
& 550 & 19 & All-sky & $50(70)$ \\
SPECS testbed & 250 & 0.17 & All-sky & $22(12)$ \\
SPIRIT & & & All-sky & $8.1(1.6)$ \\
Note that the val & & 4 & $\sim 10^{-5}\left(\sim 10^{-3}\right)$ \\
\hline
\end{tabular}

Note that the values listed for Planck Surveyor apply to an all-sky survey. Another instrument under development, which has not published detailed performance estimates is the 50-200- $\mu \mathrm{m}$ sky survey from the Japanese ASTRO-F/IRIS satellite sky survey.

groups involved in deep mm/submm surveys (Barger et al., 1999a; Eales et al., 2000; Carilli et al., 2001; Scott et al., 2002; Webb et al., 2002b) is reflected in the relatively few papers describing the individual multi-waveband properties of the almost 200 galaxies detected. The most sensitive follow-up observations are required in the near-IR, radio and optical wavebands to identify and study them (Frayer et al., 2000; Ivison et al., 2001), that is very faint detection thresholds of order $10 \mu \mathrm{Jy}$ at $1.4-\mathrm{GHz}, K \simeq 23$ and $B \gg 26$ respectively. Much more time has been devoted to multi-waveband follow-up observations than was spent on the initial submm detections. Typical examples of the submm population can be detected in imaging-mode SCUBA observations in about $10 \mathrm{~h}$ of integration. However, at least $2 \mathrm{~h}$ of near-IR observations at the 10-m Keck telescope and about $24 \mathrm{~h}$ of integration at the VLA are then required in order to find likely counterparts to typical submm galaxies. The advantage of the VLA radio observations over those at optical and near-IR wavelengths is the very large field of view, which allows many galaxies to be detected simultaneously. The very brightest optical counterparts to submm galaxies can be identified spectroscopically in about $7 \mathrm{~h}$ of integration using 
Table 3

References to instruments listed in Tables 1 and 2.

\begin{tabular}{ll} 
Name & Information \\
\hline SCUBA & Holland et al. (1999) \\
MAMBO & Kreysa et al. (1998) \\
SCUBA-II & www.jach.hawaii.edu/JACpublic/JCMT/ \\
Continuum_observing/SCUBA-2/home.html \\
SOFIA & Davidson et al. (1999); sofia.arc.nasa.gov \\
BOLOCAM & Glenn et al. (1998); www-lmt.phast.umass.edu/ins/ \\
BLAST & continuum/bolocam.html \\
SMA & Devlin (2001); www.hep.upenn.edu/blast \\
ALMA & Ho (2000); sma2.harvard.edu \\
SKA & Wootten (2001); www.alma.nrao.edu \\
Herschel-SPIRE & www.nfra.nl/skai \\
Herschel-PACS & www.ssd.rl.ac.uk/spire \\
SIRTF-MIPS \& IRAC & pacs.ster.kuleuven.ac.be \\
Planck Surveyor & sirtf.caltech.edu \\
SPECS / SPIRIT & astro.estec.esa.nl/Planck \\
\hline
\end{tabular}

4-m class telescopes (Ivison et al., 1998) and higher-quality spectra can be obtained in a comparable time using 8-m class telescopes (see the results of a 5-h integration using the UVES spectrograph at the European Southern Observatory (ESO) VLT by Vernet and Cimatti, 2001). In all cases, identifying a plausible counterpart, where possible, is only a first step; finding a redshift for these typically faint, red galaxies is much more challenging. In this context, the unusual sensitivity of submm surveys to the most distant galaxies is almost a drawback, making it very hard to detect a complete sample of submm galaxies at other wavelengths.

Key questions for understanding submm galaxies in the future include:

What are the properties of typical submm galaxies in other wavebands, and what is their relationship to other high-redshift galaxy samples? The submmselected galaxies appear to a diverse mixture of types, including bright merging systems (Ivison et al., 1998a, 2000a, 2001), optical QSOs (Kraiberg Knudsen et al., 2001), EROs with $K<20$ (Smail et al., 1999, 2002; Gear et al., 2000; Lutz et al., 2001), and much fainter IR-detected galaxies (Frayer et al., 2000), 
which may also turn out to have very red colors. It seems that the overlap between the $850-\mu \mathrm{m}$ submm galaxy population and both the LBGs and faint Chandra X-ray sources is small. Note that some of this apparent diversity is sure to be due to the very wide redshift distribution of the submm galaxies.

What is the redshift distribution of the submm galaxies? Models of the evolution of submm galaxies that do not grossly violate basic observational constraints on the source counts and cosmic background radiation are easy to generate. However, it is vital to predict a plausible redshift distribution, with only a small fraction at redshifts less than unity, and a probable median redshift of at least 2-3. It is easy to generate a redshift distribution that is biased too high. The observational determination of a redshift distribution for a welldefined sample of submm galaxies remains a crucial goal. This will be easy with ALMA. In the meantime, concerted and time-consuming campaigns of optical and near-IR spectroscopy will pay off gradually, while observations of cm-wave megamasers and the development of wide-band $\mathrm{mm}$ - and cm-wave spectrometers may offer alternative routes. The forthcoming (sub)mm interferometers CARMA and SMA, and developments of the IRAM PdBI will also provide accurate positions and some $\mathrm{CO}$ redshifts for submm galaxies.

What are the details of the astrophysics responsible for the luminosity of the submm galaxies? This is very important, as the submm galaxies appear to be signposts to some of the most luminous and violent phases of galaxy evolution, and could be associated with the formation of the bulk of galactic bulges, elliptical galaxies and supermassive black holes (Lilly et al., 1999; Dunlop, 2001). Whether these galaxies are formed in a single event, or as a series of lesser bursts, is a key question for our understanding of the process of galaxy formation and evolution. Detailed comparisons of the luminosity derived from dust continuum emission, the dynamical mass inferred from molecular line profiles, the evolved stellar mass inferred from near-IR observations, and the spatial extent of the activity from various high-resolution observations will all be important for disentangling the complex astrophysics of these systems.

When can submm instruments be used to resolve and study high-redshift galaxies in detail? This is already practical given enough observing time at the OVRO MMA and the IRAM PdBI. The CARMA and SMA interferometers will soon have important roles to play in these studies. In about 10 years, ALMA will provide the first real chance to detect and study galaxies rapidly and in great detail using submm observations alone. Luminosities, redshifts, dynamical masses and metallicities could all be determined without needing to resort to radio, optical and near-IR observations as a matter of course. However, because ALMA has a relatively small field of view, the most efficient survey strategy may be to detect large numbers of galaxies using wide-field mm/submm cameras like BOLOCAM, SCUBA-II and their successors on single-antenna 10-50-m aperture survey telescopes, and the Herschel 
and Planck Surveyor space missions, and then use ALMA to provide detailed images and spectra of all the detected galaxies.

What is the fundamental limit to making submm observations of distant galaxies? Submm observations rely on the presence of metals, in the form of molecular gas or dust grains in order to detect galaxies. While submm radiation is able to travel unattenuated across the Universe from prior to the epoch of reionization, it is possible that a large fraction of pre-reionization 'first-light' sources are insufficiently dusty and metal rich to be detectable as continuum sources. Low-metallicity galaxies should be detectable by fine-structure $\mathrm{C}$ and O far-IR line emission, however. It would be tremendously exciting to see the birth of the first metal-enriched dusty systems with ALMA, and so perhaps to determine directly the redshift limit for submm surveys. Of course, even if this were possible, ALMA would still have a long and fruitful career studying the detailed astrophysics of galaxies out to and beyond redshift 5, while the search for the most primitive galaxies in the second and third decades of the century is taken up by space-based mid-IR interferometers and the SKA radio telescope (see Fig. 8).

Submm observations of the distant Universe are a new tool for probing the earliest and most dramatic stages of the evolution of galaxies. Over the years to come, the capabilities of submm-wave observatories, and our understanding of the Universe in this new window, should continue to advance dramatically.

\section{Acknowledgments}

This work is heavily based on results obtained from the SCUBA Lens Survey. The following have all been involved with aspects of the SCUBA lens survey: Lee Armus, Amy Barger, Jocelyn Bezecourt, Leo Blitz, Len Cowie, John Davies, Alastair Edge, Aaron Evans, Andy Fabian, Allon Jameson, Tom Kerr, Jean-Francois Le Borgne, Malcolm Longair, Leo Metcalfe, Glenn Morrison, Frazer Owen, Naveen Reddy, Nick Scoville, Genevieve Soucail, Jack Welch, Mel Wright and Min Yun. We thank the staff of the JCMT for operating and the UK ATC for providing SCUBA.

We thank Omar Almaini, Vicki Barnard, Frank Bertoldi, Jamie Bock, Chris Carilli, Helmut Dannerbauer, Darren Dowell, Steve Eales, Jason Glenn, Sunil Golwala, Dean Hines, Kate Isaak, Kirsten Kraiberg Knudsen, Attila Kovacs, Andrew Lange, Simon Lilly, Ole Möller, Priya Natarajan, Max Pettini, Tom Phillips, Kate Quirk, Enrico Ramirez-Ruiz, Nial Tanvir, Neil Trentham, Paul van der Werf, the editor Marc Kamionkowski, an anonymous referee and Roberta Bernstein for useful conversations and comments on the manuscript. 
AWB was supported in Cambridge by the Raymond and Beverly Sackler Foundation as part of the Foundation's Deep Sky Initiative Program at the IoA. IRS is supported by the Leverhulme Trust and the Royal Society. JPK is supported by CNRS. Full references and acknowledgement to the instruments and telescopes used in this research can be found in Smail et al. (2002). This research has made use of the NASA/IPAC Extragalactic Database (NED) which is operated by the Jet Propulsion Laboratory, California Institute of Technology, under contract with the National Aeronautics and Space Administration.

\section{References}

[1] T. Abel, M. L. Norman and P. Madau, ApJ 523 (1999) 66.

[2] K. L. Adelberger and C. C. Steidel, ApJ 544 (2000) $218(*)$.

[3] D. Alloin, S. Guilloteau, R. Barvainis, R. Antonucci and L. Tacconi, A\&A 321 (1997) 24.

[4] O. Almaini, A. Lawrence and B. J. Boyle, MNRAS 305 (1999) L59.

[5] O. Almaini et al., MNRAS submitted (2002) astro-ph/0108400.

[6] B. Altieri et al., A\&A 343 (1999) L65 (*).

[7] P. B. Alton, E. M. Xilouris, S. Bianchi, J. Davies and N. Kylafis, A\&A 356 (2000) 795.

[8] P. B. Alton, J. Lequeux, S. Bianchi, D. Churches, J. Davies and F. Combes, A\&A 366 (2001) 451.

[9] P. Andreani and A. Franceschini, MNRAS 283 (1996) 85.

[10] E. N. Archibald, J.S. Dunlop, D. H. Hughes, S. Rawlings, S. A. Eales and R. J. Ivison, MNRAS 323 (2001) 417.

[11] E. N. Archibald, J. S. Dunlop, R. Jimenez, A. C. S. Friaca, R. J. McLure and D. H. Hughes, ApJ submitted (2002) astro-ph/0108122.

[12] C. Armand, B. Milliard and J. M. Deharveng, A\&A 284 (1994) 12.

[13] S. Arnouts, S. D’Odorico, S. Christiani, S. Zaggia, A. Fontana and E. Giallongo, A\&A 341 (1999) 641.

[14] A. J. Baker, D. Lutz, R. Genzel, L. J. Tacconi and M. D. Lehnert, A\&A 372 (2001) L37.

[15] X. Barcons et al., A\&A, 382 (2002) 522.

[16] A. J. Barger, L. L. Cowie, D. B. Sanders, E. Fulton, Y. Taniguchi, Y. Sato, K. Kawara and H. Okuda, Nature 394 (1998) 248. 
[17] A. J. Barger, L. L. Cowie and D. B. Sanders, ApJ 518 (1999a) L5 (*).

[18] A. J., Barger, L. L. Cowie, I. Smail, R. J. Ivison, A. W. Blain and J.-P. Kneib, AJ 117 (1999b) 2656.

[19] A. J. Barger, L. L. Cowie and E. A. Richards, AJ 119 (2000) 2092.

[20] A. J. Barger et al., ApJ 560 (2001) L23.

[21] V. E. Barnard and A. W. Blain, MNRAS (2002) submitted.

[22] V. E. Barnard et al., MNRAS (2002) submitted.

[23] R. Barvainis and R. J. Ivison, ApJ submitted (2002) astro-ph/0201424.

[24] C. M. Baugh, A. J. Benson, S. Cole, C. S. Frenk and C. G. Lacey, review preprint (2001) astro-ph/0103136.

[25] M. W. Bautz et al., ApJ 543 (2000) L119.

[26] K. Bekki, Y. Shioya and I. Tanaka, ApJ 520 (1999) L99.

[27] D. J. Benford, P. Cox, A. Omont, T. G. Phillips and R. G. McMahon, ApJ 518 (1999) L65.

[28] D. J. Benford, B. Maffei, S. H. Moseley, F. Pajot, T. G. Phillips, C. Rioux and R. A. Shafer, AAS 198 (2001) 0510.

[29] A. J. Benson, C. S. Frenk, C. M. Baugh, S. Cole and C. G. Lacey, MNRAS 327 (2001) 1041.

[30] E. Berger, S. R. Kulkarni and D. A. Frail, ApJ 560 (2001) 652

[31] R. A. Bernstein, in A. J Bunker and W. J. M. van Breughel eds. The Hy-Redshift Universe, ASP Conf Series Vol. 193 (1999) p. 487.

[32] R. A. Bernstein, W. L. Freedman and B. F. Madore, ApJ 571 (2002) 56.

[33] E. Bertin, M. Dennefeld and M. Moshir, A\&A 323 (1997) 685.

[34] F. Bertoldi et al., A\&A 360 (2000) 92.

[35] F. Bertoldi, K. M. Menten, E. Kreysa, C. L. Carilli and F. Owen, in 'Highlights of Astronomy' 12 (2001) PASP, astro-ph/0010553.

[36] A. W. Blain, MNRAS 283 (1996) 1340.

[37] A. W. Blain, MNRAS 290 (1997) 553.

[38] A. W. Blain, MNRAS 297 (1998) 511.

[39] A. W. Blain, MNRAS, 304 (1999a) 669.

[40] A. W. Blain, in R. J. Weymann et al. eds. Photometric Redshifts and High Redshift Galaxies, ASP Conf. Series Vol. 191 (1999b) p. 255 astro-ph/9906141. 
[41] A. W. Blain, in J. Tran Thanh Van, Y. Mellier and M. Moniez eds. 'Cosmological Physics with Gravitational Lensing' EDP Sciences (2001a) p. 245, astroph/0007196.

[42] A. W. Blain, MNRAS 330 (2002) 219.

[43] A. W. Blain and M. S. Longair, MNRAS 264 (1993a) 509 (*).

[44] A. W. Blain and M. S. Longair, MNRAS 265 (1993b) L21.

[45] A. W. Blain and M. S. Longair, MNRAS 279 (1996) 847.

[46] A. W. Blain and P. Natarajan, MNRAS 312 (2000) L39.

[47] A. W. Blain and T. G. Phillips, MNRAS 333 (2002) 222.

[48] A. W. Blain, R. J. Ivison and I. Smail, MNRAS 296 (1998) L29.

[49] A. W. Blain, J.-P. Kneib, R. J. Ivison and I. Smail, ApJ 512 (1999a) L87 (*).

[50] A. W. Blain, I. Smail, R. J. Ivison and J.-P. Kneib, MNRAS 302 (1999b) 632 $(*)$.

[51] A. W. Blain, A. Jameson, I. Smail, M. S. Longair, J.-P. Kneib and R. J. Ivison, MNRAS 309 (1999c) 715.

[52] A. W. Blain, D. T. Frayer, J. J. Bock and N.Z. Scoville, MNRAS 313 (2000) 559.

[53] J. S. Bloom, S. R. Kulkarni and S. G. Djorgovski, AJ 123 (2002) 111.

[54] C. Borys, S. C. Chapman, M. Halpern and D. Scott, MNRAS 330 (2002) L65.

[55] F. H. Briggs, preprint (1999) astro-ph/9910415.

[56] D. Calzetti, L. Armus, R. C. Bohlin, A. L. Kinney, J. Koornneef and T. StorchiBergmann, ApJ 533 (2000) 682.

[57] C. L. Carilli, private communication (2001).

[58] C. L. Carilli and G. B. Taylor, ApJ 532 (2000) L95.

[59] C. L. Carilli and M. S. Yun, ApJ 513 (1999) L13 (*).

[60] C. L. Carilli and M. S. Yun, ApJ 530 (2000) 618.

[61] C. L. Carilli and A. W. Blain, ApJ 569 (2002) 605.

[62] C. L. Carilli et al., ApJ 555 (2001) 625.

[63] C.L. Carilli et al., in Deep Millimeter Surveys: Implications for Galaxy Formation and Evolution, eds. J. Lowenthal and D. Hughes, World Scientific, p 27 (2001) astro-ph/0009298.

[64] S. C. Chapman et al., MNRAS 319 (2000) 318 (*).

[65] S. C. Chapman et al., ApJ 548 (2001a) L17. 
[66] S. C. Chapman, E. A. Richards, G. F. Lewis, G. Wilson and A. J. Barger, ApJ 548 (2001b) L147.

[67] S. C. Chapman, D. Scott, C. Borys and G. G. Fahlman, MNRAS 330 (2002a) 92.

[68] S. C. Chapman, G. F. Lewis, D. Scott., C. Borys, and E. A. Richards, ApJ 570 (2002b) 557.

[69] S. C. Chapman, A, Shapley, C. Steidel and R. A. Windhorst, ApJ in press (2002c) astro-ph/0205031.

[70] S. C. Chapman, I. Smail, R. J. Ivison, G. Helou, D. Dale and G. Lagache, ApJ in press (2002d) astro-ph/0203068.

[71] R. Chary and D. Elbaz, ApJ 556 (2001) 562.

[72] S. Cole, A. Aragon-Salamanca, C.S. Frenk, J. F. Navarro and S. E. Zepf, MNRAS 271 (1994) 781.

[73] S. Cole, C. G. Lacey, C. M. Baugh and C. S. Frenk, MNRAS 319 (2000) 168.

[74] F. Combes, R. Maoli and A. Omont, A\&A 345 (1999) 369.

[75] J. J. Condon, ApJ 188 (1974) 279.

[76] J. J. Condon, ARA\&A 30 (1992) $575\left(^{*}\right)$.

[77] L.亡. Cowie, A. J. Barger and J.-P. Kneib, AJ 123 (2002) 2197.

[78] E. Daddi et al., A\&A 361 (2000) 535.

[79] D. A. Dale, G. Helou, A. Contursi, N. A. Silbermann and S. Kolhatkar, ApJ 549 (2001) 215.

[80] H. Dannerbauer, M. D. Lehnert, D. Lutz, L. Tacconi, F. Bertoldi, C. Carilli, R. Genzel and K. Menten, ApJ in press (2002) astro-ph/0201104.

[81] J. Darling and R. Giovanelli, AJ 121 (2001) 1278.

[82] J. A. Davidson, Ap\&SS 266 (1999) 35.

[83] M. J. Devlin, preprint (2001) astro-ph/0012327.

[84] O. J. Eggen, D. Lynden-Bell and A. R. Sandage, ApJ 136 (1962) 748.

[85] J. R. Deane and N. Trentham, MNRAS 326 (2001) 1467.

[86] J. E. G. Devriendt, B. Guiderdoni and R. Sadat, A\&A 350 (1999) 381.

[87] A. Dey, J. R. Graham, R. J. Ivison, I. Smail, G. S. Wright and M. C. Liu, ApJ 519 (1999) 610.

[88] H. Dole et al., A\&A 372 (2001) 364.

[89] D. L. Domingue, W. C. Keel, S. D. Ryder and R. E. White, AJ 118 (1999) 1542. 
[90] C. D. Dowell et al., AAS 198 (2001) 0509.

[91] D. Downes and P. M. Solomon, ApJ 507 (1998) 615 (*).

[92] D. Downes et al., A\&A 347 (1999a) 809.

[93] D. Downes, R. Neri, T. Wiklind, D. J. Wilner and P. A. Shaver, ApJ 513 (1999b) L1.

[94] B. T. Draine and H. M. Lee, ApJ 285 (1984) 89.

[95] B. T. Draine and A. Li, ApJ 551 (2001) 807.

[96] J.S. Dunlop, D. H. Hughes, S. Rawlings, S. A. Eales and M. J. Ward, Nature 370 (1994) 347.

[97] J.S. Dunlop, in Deep millimeter surveys, J. Lowenthal and D. Hughes eds. World Scientific, p. 11 (2001).

[98] L. Dunne, S. Eales, M. Edmunds, R. Ivison, P. Alexander and D. L. Clements, MNRAS 315 (2000) $115(*)$.

[99] L. Dunne and S. Eales, MNRAS 327 (2001) 697.

[100] L. Dunne, D. L. Clements and S. A. Eales, MNRAS 319 (2001) 813.

[101] E. Dwek and R. Arendt, ApJ 508 (1998) L9.

[102] S. Eales, S. Lilly, W. Gear, L. Dunne, J. R. Bond, F, Hammer, O. Le Févre and D. Crampton, ApJ 515 (1999) $518(*)$.

[103] S. Eales, S. Lilly, T. Webb, L. Dunne, W. Gear, D. Clements and M. Yun, AJ 120 (2000) 2244.

[104] A. C. Edge, R. J. Ivison, I. Smail, A. W. Blain and J.-P. Kneib, MNRAS 306 (1999) 599.

[105] D. Elbaz et al., A\&A 351 (1999) L37.

[106] E. Ellingson, H. K. C. Lee, J. Bechtold and R. Elston, ApJ 466 (1996) L35.

[107] A. C. Fabian and X. Barcons, ARA\&A 30 (1992) 429.

[108] A C. Fabian, in R. Giacconi, L. Stella and S. Seiro eds. (2000), X-Ray Astronomy 2000, ASP Conf Series Vol. 234, in press, astro-ph/0103431.

[109] A. C. Fabian et al., MNRAS 315 (2000) L8.

[110] L. Ferrarese and D. Merritt, ApJ 539 (2000) L9.

[111] D. P. Finkbeiner, M. Davis and D. J. Schlegel, ApJ 544 (2000) 81.

[112] D. J. Fixsen, E. Dwek, J. C. Mather, C. L. Bennett and R. A. Shafer, ApJ 508 (1998) 123.

[113] H. Flores et al., ApJ 517 (1999) 148. 
[114] M. J. Fox et al., MNRAS 331 (2002) 839.

[115] D. A. Frail et al., ApJ 565 (2002) 829.

[116] A. Franceschini, L. Danese, G. de Zotti and C. Xu, MNRAS 233 (1988) 175.

[117] A. Franceschini, L. Toffolatti, P. Mazzei, L. Danese and G. de Zotti, A\&AS 89 (1991) 285.

[118] A. Franceschini, in I. Perez-Fournon, M. Balcells, F. Moreno-Insertis and F. Sanchez eds., Galaxies at High Redshift, Cambridge University Press, in press (2002) astro-ph/0009121.

[119] A. Franceschini, D. Fadda, C. Cesarsky, D. Elbaz, H. Flores and G. L. Granato, ApJ 568 (2002) 470.

[120] D. T. Frayer et al., AJ 113 (1997) 562.

[121] D. T. Frayer, N.Z. Scoville, M. Yun, A.S. Evans, I. Smail, A. W. Blain and J.-P. Kneib, ApJ 506 (1998) L7 (*).

[122] D. T. Frayer et al., ApJ 514 (1999) L13.

[123] D. T. Frayer, I. Smail, R. J. Ivison and N. Z. Scoville, AJ 120 (2000) 1668.

[124] D. T. Frayer et al., AJ submitted (2002).

[125] M. Fukugita, C. J. Hogan and P. J. E. Peebles, ApJ 503 (1999) 518.

[126] W. K. Gear, S. J. Lilly, J. A. Stevens, D. L. Clements, T. M. Webb, S. A. Eales and L. Dunne, MNRAS 316 (2000) L51.

[127] K. Gebhardt et al., ApJ 539 (2000) L13.

[128] R. Giacconi et al., ApJ in press (2002) astro-ph/0112184.

[129] R. Gispert, G. Lagache and J.-L. Puget, A\&A 360 (2000) 1.

[130] J. Glenn et al., SPIE 3357 (1998) 326.

[131] J. Glenn, private communication (2001).

[132] J. D. Goldader, G. Meurer, T. M. Heckman, M. Seibert, D. B. Sanders, D. Calzetti and C. C. Steidel, ApJ in press (2002) astro-ph/0112352.

[133] J. R. Graham and A. Dey, ApJ 471 (1996) 720.

[134] G. L. Granato, L. Danese and A. Franceschini, ApJ 460 (1996) L11.

[135] G. L. Granato, C. G. Lacey, L. Silva, A. Bressan, C. M. Baugh, S. Cole and C. S. Frenk, ApJ 542 (2000) 710.

[136] G. L. Granato, L. Silva, P. L. Monaco, P. Panuzzo, P. Salucci, G. De Zotti and L. Danese, MNRAS 324 (2001) 757.

[137] B. Guiderdoni, E. Hivon, F. R. Bouchet and B. Maffei, MNRAS 295 (1998) $877(*)$. 
[138] K. F. Gunn and T. Shanks, MNRAS submitted (2002) astro-ph/9909089.

[139] D. B. Haarsma, R. B. Partridge, R. A. Windhorst and E. A. Richards, ApJ 544 (2000) 641.

[140] P. B. Hacking and J. R. Houck, ApJS 63 (1987) 311.

[141] Z. Haiman and L. Knox, ApJ 530 (2000) 124.

[142] M. Harwit and F. Pacini, ApJ 200 (1975) 127.

[143] G. Hasinger et al., A\&AS 120 (1996) 607.

[144] M. G. Hauser et al., ApJ 508 (1998) 25.

[145] M. G. Hauser and E. Dwek, ARA\&A vol. 39 (2001) $249\left(^{*}\right)$.

[146] T. T. Helfer, in J. Mangum ed., Imaging at Radio through Submillimeter Wavelengths, ASP Conf. Series Vol. 217 (2000) p. 25.

[147] G. Helou and C. A. Beichman, ESA SP-314 (1990) 117.

[148] R. H. Hildebrand, QJRAS 24 (1983) 267.

[149] P. T.P. Ho, in J. Mangum ed., Imaging at Radio through Submillimeter Wavelengths, ASP Conf. Series Vol. 217 (2000) p. 227.

[150] D. W. Hogg, AJ 121 (2001) 1336.

[151] W. Holland et al., MNRAS 303 (1999) 659.

[152] D. J. Hollenbach and A. G. G. M. Tielens, ARA\&A 35 (1997) 179.

[153] A. E. Hornschemeier et al., ApJ 541 (2000) 49.

[154] E. Hu and S. E. Ridgway, AJ 107 (1994) 1303.

[155] D. Hughes, in M. N. Bremer, P.P. van der Werf, H. J. A. Röttgering and

C. L. Carilli eds., Cold Gas at High Redshift, Kluwer (1996) p.311.

[156] D. H. Hughes, J.S. Dunlop and S. Rawlings, MNRAS 289 (1997) 766.

[157] D. Hughes et al., Nature 394 (1998) $241\left(^{*}\right)$.

[158] E. Hummel, A\&A 160 (1986) L1.

[159] M. J. Irwin, R. A. Ibata, G. F Lewis and E. J. Totten, ApJ 505 (1998) 529.

[160] K. G. Isaak, R. G. McMahon, R. E. Hills and S. Withington, MNRAS 369 (1994) L28.

[161] K. G. Isaak et al., MNRAS 329 (2002) 149.

[162] R. J. Ivison, in J. Tran Thanh Van, Y. Mellier and M. Moniez eds. 'Cosmological Physics with Gravitational Lensing' (2001), EDP Sciences, p. 233. 
[163] R. J. Ivison, I. Smail, J.-F. Le Borgne, A. W. Blain, J.-P. Kneib, J. Bezecourt,

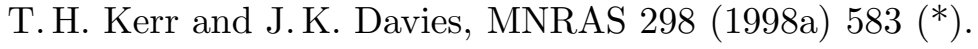

[164] R. J. Ivison et al., ApJ 494 (1998b) 211.

[165] R. J. Ivison, I. Smail, A. J. Barger, J.-P. Kneib, A. W. Blain, F. N. Owen, T. H. Kerr and L. L. Cowie, MNRAS 315 (2000a) 209.

[166] R. J. Ivison, J. S. Dunlop, I. Smail, A. Dey, M. C. Liu and J. R. Graham, ApJ $542(2000 \mathrm{~b}) 27$.

[167] R. J. Ivison, I. Smail, D. T. Frayer, J.-P. Kneib and A. W. Blain, ApJ 561 (2001) L45.

[168] R. J. Ivison et al., MNRAS submitted (2002).

[169] A. Jameson, PhD thesis (2000) University of Cambridge.

[170] M. Juvela, K. Mattila and D. Lemke, A\&A 360 (2000) 813.

[171] G. Kauffmann and S. D. M. White, MNRAS 261 (1993) 921.

[172] K. Kawara et al., A\&A 336 (1998) L9 (*).

[173] C. Kiss, P. Abraham, U. Klaas, M. Juvela and D. Lemke, A\&A 380 (2001) 388.

[174] J.-P. Kneib et al., A\&A submitted (2002).

[175] J. Kormendy and D. B. Sanders, ApJ 325 (1988) 74.

[176] K. Kraiberg Knudsen, P. P. van der Werf and W. Jaffe, in Deep millimeter surveys, J. Lowenthal and D. Hughes eds. World Scientific, p. 168 (2001) astro$\mathrm{ph} / 0009024$.

[177] E. Kreysa et al., SPIE 3357 (1998) 319.

[178] M. Krumholz, S. E. Thorsett and F. A. Harrison, ApJ 506 (1998) L81.

[179] G. Lagache and J. L. Puget, A\&A 355 (2000) L17.

[180] G. Lagache, A. Abergel, F. Boulanger, F.-X. Desert and J.-L. Puget, A\&A 344 (2000a) 322 .

[181] G. Lagache, L. M. Haffner, R. J. Reynolds and S. L. Tufte, A\&A 354 (2000b) 247.

[182] M. Lampton, S. Bowyer and J. M. Deharveng (1990), in S. Bowyer S. and C. Leinert eds., The galactic and extragalactic background radiation, Proc. IAU 139, Kluwer, p. 449.

[183] O. Laurent, I. F. Mirabel, V. Charmandaris, P. Gallais, S. C. Madden, M. Sauvage, L. Vigroux and C. Cesarsky, A\&A 359 (2000) 887.

[184] G. F. Lewis, S. C. Chapman, R. A. Ibata, M. J. Irwin and E. J. Totten, ApJ 505 (1998) L1. 
[185] S. J. Lilly, O. Le Fèvre, F. Hammer and D. Crampton, ApJ 460 (1996) L1.

[186] S. J. Lilly, S. A. Eales, W. K. P. Gear, F. Hammer, O. Le Fèvre, D. Crampton, J. R. Bond and L. Dunne, ApJ 518 (1999) 641.

[187] M. J. D. Linden-Vornle et al., A\&A 359 (2000) 51.

[188] U. Lisenfeld, K. G. Isaak and R. E. Hills, MNRAS 312 (2000) 433.

[189] G. W. Lockwood, ApJ 160 (1970) L47.

[190] M. S. Longair, in B. L. Dingus et al. eds., AIP Conference Proceedings (2000) vol. 516, p. 3.

[191] D. Lutz et al., A\&A 378 (2001) 70.

[192] M. Magliocchetti, L. Moscardini, G. de Zotti, G. L. Granato and L. Danese, MNRAS 325 (2001) 1553.

[193] J. Magorrian et al., AJ 115 (1998) 2285.

[194] M. A. Malkan and F. W. Stecker, ApJ 496 (1998) 13.

[195] M. A. Malkan and F. W. Stecker, ApJ 555 (2001) 641.

[196] S. Masi et al., ApJ 553 (2001) 93.

[197] J. C. Mather et al., ApJ 420 (1994) 439.

[198] J. C. Mather et al., SPECS definition preprint (1998) astro-ph/9812454.

[199] H. Matsuhara et al., A\&A 361 (2000) 407.

[200] L. Metcalfe, private communication (2001).

[201] G. R. Meurer, T. M. Heckman and D. Calzetti, ApJ 521 (1999) 183.

[202] J. C. Mihos and L. Hernquist, ApJ 464 (1996) 641.

[203] C. Mihos, preprint (2000) astro-ph/9903115.

[204] I. F. Mirabel et al., A\&A 333 (1998) L1.

[205] N. R. Mohan, A. Cimatti, H. J. A Röttgering, P. Andreani, P. Severgnini, R. P. J. Tilanus, C. L. Carilli and S. A. Stanford, A\&A 383 (2002) 440.

[206] J. Murthy, D. Hall, M. Earl, R. C. Henry and J. B. Holberg, ApJ 522 (1999) 904.

[207] R. F. Mushotzky, L. L. Cowie, A. J. Barger and K. A. Arnaud, Nature 404 (2000) 459.

[208] S. Oliver, M. Rowan-Robinson and W. Saunders, MNRAS 256 (1992) P15.

[209] A. Omont, P. Cox, F. Bertoldi, R. G. McMahon, C. Carilli and K. G. Isaak, A\&A 374 (2001) 371. 
[210] M. J. Page, J. A. Stevens, J. P. D. Mittaz and F. J. Carreras, Science 294 (2002) 2516.

[211] R. B. Partridge and P. J. E. Peebles, ApJ 147 (1967) 868.

[212] R. B. Partridge, E. A. Richards, E. B. Fomalont, K. I. Kellerman and R. A. Windhorst, ApJ 483 (1997) 38.

[213] P. Papadopoulos, R. Ivison, C. Carilli and G. Lewis, Nature 409 (2001) 58.

[214] J. A. Peacock et al., MNRAS 318 (2000) 535.

[215] C. Pearson, MNRAS 325 (2001) 1511.

[216] C. Pearson and M. Rowan-Robinson, MNRAS 283 (1996) 174.

[217] P. J.E. Peebles, Principles of Physical Cosmology (1993) Princeton, New Jersey.

[218] M. Pettini, M. Kellogg, C. C. Steidel, M. Dickinson, K. L. Adelberger and M. Giavalisco, ApJ 508 (1998) 539.

[219] M. Pettini, C. C. Steidel, K. L. Adelberger, M. Dickinson and M. Giavalisco, ApJ 528 (2000) 96.

[220] M. Pettini, A. E. Shapley, C. C. Steidel, J.-G. Cuby, M. Dickinson, A. F. M. Moorwood, K. L. Adelberger and M. Giavalisco, ApJ 554 (2001) 981.

[221] D. Pierce-Price et al., ApJ 545 (2000) L121.

[222] M. Pierre et al., A\&A 372 (2001) L45.

[223] L. Pozzetti, P. Madau, G. Zamorani, H. Ferguson and A. Bruzual, MNRAS 298 (1998) 1133.

[224] R. S. Priddey and R. G. McMahon, MNRAS 324 (2001) L17.

[225] J.-L. Puget, A. Abergel, J.-P. Bernard, F. Boulanger, W. B. Burton, F.X. Désert and D. Hartmann, A\&A 308 (1996) L5 (*).

[226] Puget J.-L. et al., A\&A 345 (1999) 29.

[227] E. Ramirez-Ruiz, N. Trentham and A. W. Blain, MNRAS 329 (2002) 465.

[228] W. T. Reach et al., ApJ 451 (1995) 188.

[229] M. W. Regan, M. D. Thornley, T. T. Helfer, K. Sheth, T. Wong, S. N. Vogel, L. Blitz and D. C. J. Bock, ApJ 561 (2001) 218.

[230] E. A. Richards, ApJ 533 (2000) 611.

[231] D. Rigopoulou, H. W. W. Spoon, R. Genzel, D. Lutz, A. F. M. Moorwood and Q. D. Tran, AJ 118 (1999) 2625.

[232] M. Rowan-Robinson et al., Nature 351 (1991) 719. 
[233] M. Rowan-Robinson et al., MNRAS 289 (1997) 490.

[234] M. Rowan-Robinson, ApJ 549 (2001) 745.

[235] M. Rowan-Robinson, MNRAS 316 (2000) 885.

[236] D. Rusin, AAS 197 (2001) 6704.

[237] K. Sakamoto, N. Z. Scoville, M. S. Yun, M. Crosas, R. Genzel and L. Tacconi, ApJ 514 (1999) 68.

[238] D. B. Sanders, Ap\&SS 266 (1999) 331.

[239] D. B. Sanders, preprint (2001) astro-ph/0109138.

[240] D. B. Sanders and F. Mirabel, ARA\&A 34 (1996) $749\left(^{*}\right)$.

[241] D. B. Sanders, B. T. Soifer, J. H. Elias, G. Neugebauer and K. Matthews, ApJ 328 (1988) L35.

[242] W. Saunders, M. Rowan-Robinson, A. Lawrence, G. Efstathiou, N. Kaiser, R.S. Ellis and C.S. Frenk, MNRAS 242 (1990) 318 (*).

[243] P. A. G. Scheuer, MNRAS 166 (1974) 329.

[244] D. J. Schlegel, D. P. Finkbeiner and M. Davis, ApJ 500 (1998) 525 (*).

[245] P. Schneider, J. Ehlers and E. E. Falco, Gravitational Lenses (1992) Springer Verlag, Berlin.

[246] D. Scott and M. White, A\&A 346 (1999) 1.

[247] D. Scott et al., A\&A 357 (2000) L5.

[248] S. E. Scott et al., MNRAS 331 (2002) 817.

[249] S. Seitz, R. P., Saglia, R. Bender, U. Hopp, P. Belloni and B. Ziegler, MNRAS 298 (1998) 945.

[250] S. Serjeant et al., MNRAS 322 (2001) 262.

[251] I. Smail, R. J. Ivison and A. W. Blain, ApJ 490 (1997) L5 (*).

[252] I. Smail, R. J. Ivison, A. W. Blain and J.-P. Kneib, ApJ 507 (1998a) L21.

[253] I. Smail, A. C. Edge, R. S. Ellis and R. D. Blandford, MNRAS 293 (1998b) 124.

[254] I. Smail et al., MNRAS 308 (1999) 1061.

[255] I. Smail, R. J. Ivison, F. N. Owen, A. W. Blain and J.-P. Kneib, ApJ 528 (2000) $612(*)$

[256] I. Smail, R. J. Ivison, A. W. Blain and J.-P. Kneib, MNRAS 331 (2002) 495 $(*)$.

[257] H. E. Smith, C. J. Lonsdale, C. L. Lonsdale and P. J. Diamond, ApJ 493 (1998) L17. 
[258] G. P. Smith, T. Treu, R. Ellis, I. Smail, J.-P. Kneib and B. L. Frye, ApJ 562 (2001) 635.

[259] I. A. Smith et al., A\&A 347 (1999) 92.

[260] I. A. Smith, R. P. J. Tilanus, R. A. M. J. Wijers, N. Tanvir, P. Vreeswijk, E. Rol and C. Kouveliotou, A\&A 380 (2002) 81.

[261] B. T. Soifer et al., AJ 320 (1987) 238.

[262] B. Soifer and G. Neugebauer, AJ 101 (1991) 354.

[263] R. S. Somerville, J. R. Primack and S. M. Faber, MNRAS 320 (2001) 504.

[264] G. Soucail, J.-P. Kneib, J. Bézecourt, L. Metcalfe, B. Altieri and J.F. Le Borgne, A\&A 343 (1999) L70.

[265] T. Stanev and A. Franceschini, ApJ 494 (1998) L159.

[266] C. C. Steidel, M. Giavalisco, M. Pettini, M. Dickinson, K. L. Adelberger, ApJ 624 (1996) L17.

[267] C. C. Steidel, K. L. Adelberger, M. Giavalisco, M. Dickinson and M. Pettini, ApJ 519 (1999) $1\left(^{*}\right)$.

[268] M. Stickel et al., A\&A 336 (1998) 116.

[269] J. C. Tan, J. Silk and C. Balland, ApJ 522 (1999) 579.

[270] D. Thompson et al., ApJ 523 (1999) 100.

[271] L. Toffolatti et al., MNRAS 297 (1998) 117.

[272] G. Toller, H. Tanabe and J. L. Weinberg, A\&A 188 (1987) 24.

[273] T. Totani, Y. Yoshii, F. Iwamuro, T. Maihara and K. Motohara, ApJ 559 (2001) 592.

[274] R. Townsend, R. Ivison, I. Smail, A. Blain and D. Frayer, MNRAS 328 (2001) L19.

[275] Q. D. Tran et al., ApJ 552 (2001) 527.

[276] N. Trentham and A. W. Blain, MNRAS 323 (2001) 547.

[277] N. Trentham, A. W. Blain and J. Goldader, MNRAS 305 (1999) 61.

[278] P. P. van der Werf, K. Kraiberg Knudsen, I. Labbé and M. Franx (2002) in I. van Bemmel, B. Wilkes and P. Barthel eds., Elsevier New Astronomy Reviews, in press, astro-ph/0011217.

[279] P. P. van der Werf and K. Kraiberg Knudsen, private communication (2001).

[280] J. Vernet and A. Cimatti, A\&A 380 (2001) 409.

[281] H. J. Völk, A\&A 218 (1988) 67. 
[282] E. Waxman and A. Loeb, ApJ 545 (2000) L11.

[283] T. M. Webb, S. A. Eales, S. J. Lilly, L. Dunne, W. K. Gear, H. Flores and M. Yun, ApJ submitted (2002a) astro-ph/0201180.

[284] T. M. Webb et al., ApJ submitted (2002b) astro-ph/0201181.

[285] S. D. M. White and C.S. Frenk, ApJ 379 (1991) 52.

[286] R. E. Williams et al., AJ 112 (19956) 1335.

[287] R. J. Wilman, A. C. Fabian and P. Ghandhi, MNRAS 318 (2000) L11.

[288] D. J. Wilner and M. C. H. Wright, ApJ 488 (1997) 67.

[289] C. D. Wilson, N. Scoville, S. C. Madden and V. Charmandaris, ApJ 542 (2000) 120.

[290] A. N. Witt, H. A. Thronson and J. M. Capuano, ApJ 393 (1992) 611.

[291] A. Wootten ed., Science with the Atacama Large Millimeter Array, ASP Conf. Series Vol. 235 (2001) ASP: San Francisco.

[292] E. L. Wright and B. D. Johnson, ApJ submitted (2002) astro-ph/0107205

[293] C. Xu, ApJ 541 (2000) 134.

[294] L. Yan et al., AJ 120 (2000) 575.

[295] M. S. Yun and C. L. Carilli, ApJ 568 (2002) 88.

[296] M. S. Yun, N. A. Reddy and J. J. Condon, ApJ 554 (2001) 803 (*). 